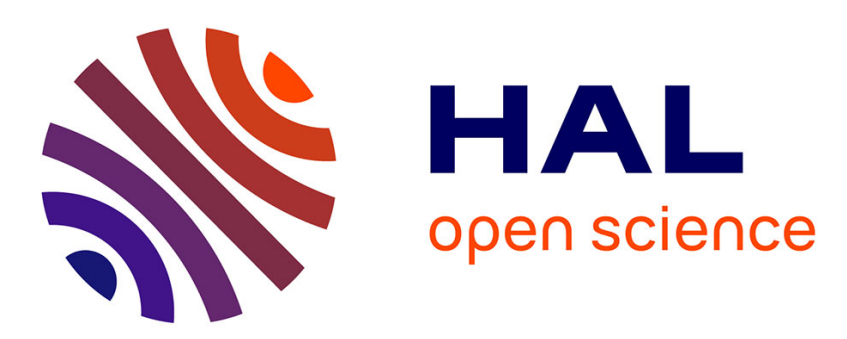

\title{
Definable relations and first-order query languages over strings
}

Michael Benedikt, Leonid Libkin, Thomas Schwentick, Luc Segoufin

\section{To cite this version:}

Michael Benedikt, Leonid Libkin, Thomas Schwentick, Luc Segoufin. Definable relations and firstorder query languages over strings. Journal of the ACM (JACM), 2003, 50 (5), pp.694-751. 10.1145/876638.876642. hal-02796572

\section{HAL Id: hal-02796572 \\ https://hal.inria.fr/hal-02796572}

Submitted on 5 Jun 2020

HAL is a multi-disciplinary open access archive for the deposit and dissemination of scientific research documents, whether they are published or not. The documents may come from teaching and research institutions in France or abroad, or from public or private research centers.
L'archive ouverte pluridisciplinaire HAL, est destinée au dépôt et à la diffusion de documents scientifiques de niveau recherche, publiés ou non, émanant des établissements d'enseignement et de recherche français ou étrangers, des laboratoires publics ou privés. 


\title{
Definable Relations and First-Order Query Languages over Strings
}

\author{
Michael Benedikt \\ Bell Laboratories
}

\author{
Leonid Libkin \\ University of Toronto
}

\author{
Thomas Schwentick \\ University of Marburg
}

\author{
Luc Segoufin \\ INRIA
}

\begin{abstract}
We study analogs of classical relational calculus in the context of strings. We start by studying string logics. Taking a classical model-theoretic approach, we fix a set of string operations and look at the resulting collection of definable relations. These form an algebra - a class of $n$-ary relations for every $n$, closed under projection and Boolean operations. We show that by choosing the string vocabulary carefully, we get string logics that have desirable properties: computable evaluation and normal forms. We identify five distinct models and study the differences in their model-theory and complexity of evaluation. We identify a subset of these models which have additional attractive properties, such as finite VC dimension and quantifier elimination.

Once you have a logic, the addition of free predicate symbols gives you a string query language. The resulting languages have attractive closure properties from a database point of view: while SQL does not allow the full composition of string pattern-matching expressions with relational operators, these logics yield compositional query languages that can capture common string-matching queries while remaining tractable. For each of the logics studied in the first part of the paper, we study properties of the corresponding query languages. We give bounds on the data complexity of queries, extend the normal form results from logics to queries, and show that the languages have corresponding algebras expressing safe queries.
\end{abstract}

\section{Introduction}

In the past 40 years, various connections between logic on strings, formal languages and finite automata have been explored in great detail. The standard setting for connecting logical definability with various properties of formal languages is to represent strings over a finite alphabet $\Sigma=\left\{a_{1}, \ldots, a_{n}\right\}$ as first-order structures in the signature $\left(P_{a_{1}}, \ldots, P_{a_{n}},<\right)$, so that the structure $M_{s}$ for a string $s$ of length $k$ has the universe $\{1, \ldots, k\}$, with $<$ being the usual ordering, and $P_{a_{i}}$ being the set of the positions $l$ such that the $l$ th character in $s$ is $a_{i}$. Then a sentence $\Phi$ of some logic $\mathcal{L}$ defines a language $L(\Phi)=\left\{s \in \Sigma^{*} \mid M_{s} \models \Phi\right\}$. Two classical results on logic and language theory state that languages thus definable in monadic second-order logic (MSO) are precisely the regular languages [20], and the languages definable in first-order logic (FO) are precisely the star-free languages [54]. For a survey, see [65, 67].

An alternative approach to definability of strings, based on classical infinite model theory rather than finite model theory, dates back to the 1960s [20,19]. One considers an infinite structure $M$ consisting of $\left\langle\Sigma^{*}, \Omega\right\rangle$, where $\Omega$ is a set of functions, predicates and constants on $\Sigma^{*}$. One can then look at definable sets, those of the form $\{\vec{a} \mid M \models$ $\varphi(\vec{a})\}$, where $\varphi$ is a first-order formula in the language of $M$. A well-known result links definability with traditional formal language theory. Let $\Omega_{\mathrm{reg}}$ consist of unary functions $l_{a}, a \in \Sigma$, binary predicates $\operatorname{el}(x, y)$ and $x \preceq y$, where $l_{a}(x)=x \cdot a, \mathrm{el}(x, y)$ states that $x$ and $y$ have the same length, and $x \preceq y$ states that $x$ is a prefix of $y$. Let $\mathbf{S}_{\text {len }}$ be the model $\left\langle\Sigma^{*}, \Omega_{\mathrm{reg}}\right\rangle$ (we will explain the notation later). Then subsets of $\Sigma^{*}$ definable in $\mathbf{S}_{\text {len }}$ are precisely the regular languages [20, 19, 14]; moreover, this implies decidability of the first-order theory of $\mathbf{S}_{\text {len }}[45,14]$.

The key advantage of the "model-theoretic approach" is that one immediately gets an extension of the notion of recognizability from string languages to $n$-ary string relations for arbitrary $n$. One gets an algebra of $n$-ary string relations for every $n$, and these algebras automatically have closure under projection and product, in addition to the Boolean operations. In the case of the model $\mathbf{S}_{\text {len }}$ above, this algebra is not new: in fact, the definable $n$-ary relations are exactly the ones recognizable under a natural notion of automaton running over $n$-tuples [19,29]. We will refer to these automata-definable relations as the regular relations: the formal definition is given in subsection 3.1.1. We show here that by taking restrictions of the model $\mathbf{S}_{\text {len }}$, one gets new algebras of regular relations which behave better, in many ways, than the full algebra of recognizable relations given by $\mathbf{S}_{\text {len }}$. We introduce four such models here, and 
show that the definable sets in these models enjoy superior model-theoretic properties relative to the full algebra of recognizable relations associated with $\mathbf{S}_{\text {len }}$.

A key motivation for finding closed algebras of string relations comes from the field of databases, in particular, the study of query languages with interpreted operations $[8,10,37,50]$. String manipulation facilities have long been recognized as a critical component of a realistic database query language. In SQL, for example, the WHERE clause can contain string pattern-matching expressions, such as FACULTY. NAME LIKE 'Nyk $\frac{\circ}{\circ}$ n'. These expressions can themselves be seen as queries over string relations: the above clause, for example, can be seen as a selection performed on a projection of the FACULTY relation. While the Relational Calculus gives a satisfactory formal model for SQL queries in the absence of built-in datatypes, there has been thus far no satisfactory model that fully accounts for string queries. The lack of an adequate formal model is related to the fact that SQL restricts the interaction of string operations and relational operations in a number of ad-hoc ways: one cannot apply the LIKE operator to a subquery to build up a new query, nor can one take the product of two string expressions built with LIKE. The natural way to obtain a calculus on string relations where one can freely compose string operations and relational operators is to start with a decidable structure on strings, like those mentioned above, and extend them to query languages by adding free predicate symbols - in the same way that traditional Relational Calculus can be obtained from first-order logic over pure equality. Using this approach we see that corresponding to $\mathbf{S}_{\text {len }}$ and each of the four restricted models mentioned above, we obtain five interesting compositional query languages on strings.

The paper has two main parts. In the first part, we study definable algebras of string relations, that is, modeltheoretic structures on $\Sigma^{*}$ and definability in these structures. We focus on five structures, of which the model $\mathbf{S}_{\text {len }}$ mentioned above is the richest. In the second part of the paper, we deal with database applications, and study the corresponding query languages for string databases given by each of the five structures. This can be thought of as definability over model-theoretic structures and a finite relational database. Naturally, the results of the first part form the basis for reasoning about string query languages.

We now summarize the developments in both parts of the paper.

As mentioned above, we know that there exists $a$ regular string algebra [20, 19, 14], i.e., an algebra which exactly captures the regular sets when restricted to unary relations. An obvious question to ask, then, is whether new algebras of string relations arise through the model-theoretic approach. In particular, if we restrict the signature $\Omega$ to be less expressive than $\Omega_{\text {reg }}$, do we get new relation algebras lying within the recognizable relations?

A natural starting point would be to find a signature that captures properties of the star-free sets. Here again, a simple example leaps out: consider the signature $\Omega_{\mathrm{sf}}=\left(\preceq,\left(l_{a}\right)_{a \in \Sigma}\right)$, and let $\mathbf{S}=\left\langle\Sigma^{*}, \Omega_{\mathrm{sf}}\right\rangle$. One can easily show that the definable subsets of $\Sigma^{*}$ in $\mathbf{S}$ are exactly the star-free ones. Furthermore, we will show that the definable $n$-ary relations of this model are exactly those definable by regular prefix automata (cf. [4]) whose underlying string automata are counter-free.

Just as there is a significant difference between the complexity-theoretic behavior of regular languages and starfree languages (the latter are in $\mathrm{AC}^{0}$ whereas the former are not), we find that the model $\mathbf{S}$ is much more tractable, in terms of its model-theory and its complexity than $\mathbf{S}_{\text {len }}$. In particular, we show that $\mathbf{S}$ has quantifier-elimination in a natural relational extension, while $\mathbf{S}_{\text {len }}$ does not.

It would be tempting to think of $\mathbf{S}$ and $\mathbf{S}_{\text {len }}$ as canonical extensions of the notions of regularity and star-free to $n$-ary relations. However, we will show that in fact there are many choices for $\Omega$ that share the same one-dimensional definable sets (either star-free or regular). Furthermore, algebras of definable sets may be identical in terms of the string languages they define, but differ considerably in the $n$-ary string relations in the definable algebra. We thus say that an algebra of definable sets based on $\left\langle\Sigma^{*}, \Omega\right\rangle$, with $\Omega \subseteq \Omega_{\mathrm{reg}}$ is a regular algebra of definable sets if the subsets of $\Sigma^{*}$ in it (i.e the one-dimensional definable sets of $\left\langle\Sigma^{*}, \Omega\right\rangle$ ) are exactly the regular sets. We likewise say that the algebra based on definable sets for $\left\langle\Sigma^{*}, \Omega\right\rangle$ is a star-free algebra of definable sets if the subsets of $\Sigma^{*}$ in the algebra are exactly the star-free sets.

We then study new examples of regular and star-free definable algebras. We give an example of a star-free algebra with considerably more expressive power than the basic star-free algebra $\mathbf{S}$. This model, which we denote by $\mathbf{S}_{\text {left }}($ as it allows one to add characters on the left of a string), shares most of the desirable properties of $\mathbf{S}$ : in particular, it has quantifier-elimination in a natural language, and membership test in this algebra has low complexity.

More surprisingly, perhaps, we give examples of regular algebras (which we denote $\mathbf{S}_{\text {reg }}$ and $\mathbf{S}_{\text {reg,left }}$ ) that are strictly contained in $\mathbf{S}_{\text {len }}=\left\langle\Sigma^{*}, \Omega_{\mathrm{reg}}\right\rangle$. Although the one-dimensional sets in these algebras are still the regular 
sets, the algebra as a whole shares many of the attractive properties of the star-free languages. In particular, we give quantifier-elimination results for these algebras. In contrast to this, we present a result giving a partial answer to open question 0 in [55], which asks whether $\mathbf{S}_{\text {len }}$ itself has quantifier-elimination in a reasonable signature. We show that it does not have quantifier-elimination in any relational signature of bounded arity but does have quantifier-elimination in a signature containing binary functions.

We now turn to the second part of the paper, studying the string query languages formed from each of these models. What are some properties one would desire of a string query language? One problem faced in any work combining string pattern-matching queries with relational calculus is that pattern-matching expressions may return an infinite number of strings. This is the standard issue of safety. Previous proposals for combining relational algebra with string-matching primitives tackle this problem by identifying safe fragments of their languages, using a number of syntactic restrictions — see, e.g., [39, 42, 38, 40, 59] — but they cannot capture the safe fragment of the language syntactically. A second issue with any string query language is its expressive power. Many query languages designed in the prior literature turn out to be Turing complete, a feature that in turn makes many sorts of analysis and optimization impossible. Indeed, as noted in [40], adding just concatenation to the relational calculus already yields a query language which is Turing complete. This immediately implies that there is no effective syntax for the corresponding safe fragment [64].

In contrast to the above, we would like our languages to fulfill the following criteria:

1. Query evaluation is efficient;

2. There is effective syntax capturing safe queries;

3. There is an algebra equivalent to the language.

Hence, we consider each of our query languages with respect to these criteria. As mentioned above, we consider relational calculus, $\mathrm{RC}$, over each model defined in the first part, beginning with the weakest model, $\mathbf{S}$. The query language obtained by adding database relations to $\mathbf{S}$ captures basic SQL with simple LIKE pattern-matching and lexicographic ordering. We show that the safe fragment of this model can be effectively captured in a natural way, and prove complexity bounds for queries in this language that match the known bounds for ordinary relational calculus. $\mathrm{RC}(\mathbf{S})$ however, is unable to express certain natural queries, e.g., SELECT $a \cdot x \operatorname{FROM} R$, where $a$ is a fixed character. We contrast this to the query language $\mathrm{RC}\left(\mathbf{S}_{\text {len }}\right)$ formed over the richest model. This extension has much greater expressiveness: it enables additional operations such as trimming/adding symbols on both left and right of a string, and the SIMILAR pattern-matching for checking membership in a regular language [41]. We show that this language also satisfies criteria 2 and 3 above, but in $\mathrm{RC}\left(\mathbf{S}_{\text {len }}\right)$ one can express NP-complete and coNP-complete problems.

This leads us to the consideration of the three intermediate languages, $\operatorname{RC}\left(\mathbf{S}_{\text {left }}\right), \operatorname{RC}\left(\mathbf{S}_{\text {reg }}\right)$, and $\operatorname{RC}\left(\mathbf{S}_{\text {reg, left }}\right)$. We find that each of these languages satisfies all three of the required criteria, while considerably extending the expressive power of $\mathrm{RC}(\mathbf{S})$.

Related Work: One motivation of our approach was the study of automatic structures [48, 14], which are a subclass of recursive structures [43], and were introduced as a generalization of automatic groups [30]. In an automatic structure $M=\left\langle\Sigma^{*}, \Omega\right\rangle$, every predicate in $\Omega$ is definable by a finite automaton. More precisely, an $n$-ary predicate $P$ is given by a letter-to-letter $n$-automaton [29, 34]. These structures were also studied in [45] in connection with decidability questions for first-order theories.

It is known $[19,14]$ that a structure is automatic iff it can be interpreted in the structure $\mathbf{S}_{\text {len }}$; hence $\mathbf{S}_{\text {len }}$ is in some sense the universal automatic structure. The first part of this paper can be seen as a study of subclasses of automatic structures definable within $\mathbf{S}_{\text {len }}$ that are significantly more restrictive, and that might have stronger model-theoretic or computational properties than a rich structure like $\mathbf{S}_{\text {len }}$.

The structure $\mathbf{S}_{\text {left }}$, without the prefix relation, is useful for modeling queues and it first appeared in the verification context [16], where an algorithm for deciding existential sentences was given. That algorithm was extended to the full theory in [60], but still without the prefix relation.

On the database side, several approaches toward unifying string algebras with relational algebra have been developed in the prior literature. Most of them are based on the concatenation operator, or other operations that make logics undecidable in general. [36] studied the consequences of adding pattern-matching features to SQL. Papers [39, 42, 38] 
proposed an extension of the relational calculus with alignment logics and studied their complexity and expressive power. Without restrictions, they can define an arbitrary r.e. set [39]. Another approach was proposed in [17, 18], which considered Datalog extended with appropriate transducers for string operations, and proved a number of completeness results. In [24] arbitrary regions (substrings) can be queried; this, when coupled with relational calculus, gives the power of string concatenation. Closer to our approach, [40, 59] study the relational calculus/algebra extended with an operation for concatenating strings. [25] studies first-order logic over term algebras and extends expressive bounds and complexity results from relational calculus to this setting. But SQL-style string pattern-matching cannot be expressed in the language of [25] - indeed in this language one cannot even query for strings beginning with a fixed symbol.

The general approach to studying databases over interpreted domains is closely related to the field of constraint databases [50]. Most theory of constraint databases was done over continuous domains, typically various structures over the reals. In contrast, our results could be viewed as the theory of (finite) constraint databases over discrete domains, in particular, strings.

Organization: The paper is organized as follows. The next section gives the notation that will be used in the paper. Then we deal with definability for models on strings, in particular, quantifier elimination, bounded VC dimension and expressive power. The last part contains database applications in terms of expressiveness, data complexity and safety of the corresponding query languages. Earlier presentation of this work appeared in two conference proceedings: $[13,12]$.

\section{Notation}

Throughout the paper, $\Sigma$ denotes a finite alphabet, and $\Sigma^{*}$ the set of all finite strings over $\Sigma$. We consider a number of operations and predicates on $\Sigma^{*}$ :

- $x \cdot y$-concatenation of two strings $x$ and $y$.

- $x \preceq y-x$ is a prefix of $y$.

- $l_{a}(x), a \in \Sigma$, is $x \cdot a$ (adds last character).

- $f_{a}(x), a \in \Sigma$, is $a \cdot x$ (adds first character).

- $|x|$ is the length of string $x$.

- $x \sqcap y$ is the longest common prefix of the strings $x$ and $y$.

- $x-y-$ the string $z$ such that $y \cdot z=x$, if it exists, and $\epsilon$ otherwise.

- $x+y$, which is an alternative notation for the concatenation $y \cdot x$. Note that always $(x+y)-y=y$.

- $\operatorname{el}(x, y)$ is true iff $|x|=|y|$.

We write $w[i, j]$ to refer to the substring of a string $w$ starting from position $i$ and ending at position $j$. Here, the first position of a string has number 1, e.g., it holds that $w=w[1,|w|]$. We write $w[i]$ for $w[i, i]$.

We write $x \lessdot y$ to express that $y$ extends $x$ by exactly one symbol. Let prefix $(C)$ stand for the prefix-closure of $C$ : $\left\{s \mid s \preceq s^{\prime}, s^{\prime} \in C\right\}$. By $\downarrow(C)$ we denote $\left\{s|| s|\leq| s^{\prime} \mid, s^{\prime} \in C\right\}$.

Given a set $S$ of strings, we let $\operatorname{Tree}(S)$ be the tree (i.e. the partially-ordered structure) generated by closing $S \cup\{\epsilon\}$ under $\sqcap$. In other words, Tree $(S)$ is the poset $\langle\{x \sqcap y \mid x, y \in S \cup\{\epsilon\}\}, \prec\rangle$. (Note that for any set of strings $s_{1}, \ldots, s_{k}$, there are two indices $i, j \leq k$ such that $s_{1} \sqcap \ldots \sqcap s_{k}=s_{i} \sqcap s_{j}$.)

If $S$ is a set of strings and $w \in \Sigma^{*}$, let $\operatorname{Meet}(w, S)$ be the longest string among $\{w \sqcap u \mid u \in S\}$, let Meet_( $(w, S)$ be the element of $\operatorname{Tree}(S)$ which is the longest prefix of $\operatorname{Meet}(w, S)$, and let $\operatorname{Meet}_{+}(w, S)$ be the smallest element of $\operatorname{Tree}(S)$ for which $\operatorname{Meet}(w, S)$ is a prefix. Note that $\operatorname{Meet}_{+}(w, S)$ is well-defined (as are $\operatorname{Meet}(w, S)$ and Meet_ $(w, S))$, since $\operatorname{Meet}(w, S)$ is either a string from $\operatorname{Tree}(S)$ or it has a unique smallest extension in $\operatorname{Tree}(S)$. 
A complete tree-order description of a vector $\vec{w}$ of variables is the atomic diagram of Tree $(\vec{w})$ in the language of $\epsilon, \preceq, \sqcap$. In other words, it is a specification of all the $\preceq$ relations that hold and do not hold in Tree $(\vec{w})$.

For example, let $\vec{w}=(a, a b a, a b b b)$. Then $a b a \sqcap a b b b=a b$, and Tree $(\vec{w})$ is $\{\epsilon, a, a b, a b a, a b b b\}$. The complete tree-order description of $\vec{w}$ consists of all the $\preceq$ relations that hold among the elements of $\{\epsilon, a, a b, a b a, a b b b\}$, as well as all the $\sqcap$-relations, e.g., $a b a \sqcap a b b b=a b, a \sqcap a b a=a, a b \sqcap \epsilon=\epsilon$, etc.

We shall consider several structures on $\Sigma^{*}$. The basic one is the structure $\mathbf{S}=\left\langle\Sigma^{*}, \preceq,\left(l_{a}\right)_{a \in \Sigma}\right\rangle$. We could equivalently use unary predicates $L_{a}$, where $L_{a}(x)$ is true for strings $x$ having $a$ as last symbol. Note that in the presence of $\preceq, l_{a}$ and $L_{a}$ are interdefinable, and we thus shall use both of them.

We further consider a number of extensions of $\mathbf{S}$. In one of them characters can be added on the left as well as on the right. This structure is denoted by $\mathbf{S}_{\text {left }} \stackrel{\text { def }}{=}\left\langle\Sigma^{*}, \preceq,\left(l_{a}\right)_{a \in \Sigma},\left(f_{a}\right)_{a \in \Sigma}\right\rangle$.

Another extension, denoted by $\mathbf{S}_{\text {len }}$, adds length comparisons via the el predicate (note that using $\preceq$ and el one can express various relationships between lengths of strings, e.g. $|x|\{=, \neq,<,>\}|y|,|x|=|y|+k$ for a constant $k$, etc.). To summarize, we mainly deal with the following structures:

- $\mathbf{S}=\left\langle\Sigma^{*}, \preceq,\left(l_{a}\right)_{a \in \Sigma}\right\rangle ;$

- $\mathbf{S}_{\text {left }}=\left\langle\Sigma^{*}, \preceq,\left(l_{a}\right)_{a \in \Sigma},\left(f_{a}\right)_{a \in \Sigma}\right\rangle$;

- $\mathbf{S}_{\text {len }}=\left\langle\Sigma^{*}, \preceq,\left(l_{a}\right)_{a \in \Sigma}, \mathrm{el}\right\rangle$.

Once we consider regular algebras, we introduce two more structures; however, operations in them will be motivated by quantifier-elimination results for $\mathbf{S}$ and $\mathbf{S}_{\text {left }}$ and thus those structures will be defined later.

There is a very close connection between $\mathbf{S}_{\text {len }}$ and an extension of Presburger arithmetic. Assume that $\Sigma=\{0,1\}$. Let $\operatorname{val}(n)$, for $n \in \mathbb{N}$, be $n$ in binary, considered as a string in $\Sigma^{*}$. Let $V_{2}(n)$ be the largest power of 2 that divides $n$. Then $P \subseteq \mathbb{N}^{k}$ is definable in $\left\langle\mathbb{N},+, V_{2}\right\rangle$ iff $\left\{\left(\operatorname{val}\left(n_{1}\right), \ldots, \operatorname{val}\left(n_{k}\right)\right) \mid\left(n_{1}, \ldots, n_{k}\right) \in P\right\}$ is definable in $\mathbf{S}_{\text {len }}$ [20, 19].

Definability over $\mathbf{S}, \mathbf{S}_{\text {left }}, \mathbf{S}_{\text {len }}$. We give a few simple examples of definability over these structures.

Matching with LIKE can be expressed over $\mathbf{S}$, since definable subsets in $\mathbf{S}$ are precisely star-free languages. For example, the condition $x$ LIKE a_bo $a$ again — can be expressed by a formula $\varphi(x)$ :

$$
\exists u, v, w\left(\begin{array}{l}
u \prec v \prec w \prec x \\
\wedge \quad L_{a}(u) \wedge L_{b}(v) \wedge L_{a}(w) \\
\wedge \quad \psi_{1}(u) \wedge \psi_{3}(v) \wedge \psi_{-1}(w)
\end{array}\right),
$$

where $\psi_{1}(u), \psi_{3}(v), \psi_{-1}(w)$ say that $u, v, w$ are prefixes extending up to the first, third, and penultimate positions in the string $x$.

Another important operation expressible over $\mathbf{S}$ is the lexicographic ordering $\leq_{\text {lex }}$. Assume that $\Sigma=\left\{a_{1}, \ldots, a_{n}\right\}$ and an ordering $a_{1}<\ldots<a_{n}$ is given. The lexicographic ordering $x \leq_{\text {lex }} y$ is then expressed by:

$$
x \preceq y \vee \exists z\left(z \prec x \wedge z \prec y \wedge \bigvee_{i<j}\left(\left(l_{a_{i}}(z) \preceq x\right) \wedge\left(l_{a_{j}}(z) \preceq y\right)\right)\right) .
$$

The graph of the function $f_{a},\left\{(x, y) \mid y=f_{a}(x)\right\}$, is definable in $\mathbf{S}_{\text {len }}$ by

$$
\begin{aligned}
&|y|=|x|+1 \wedge\left(\exists w \prec y|w|=1 \wedge L_{a}(w)\right) \\
& \wedge \quad \forall z \prec x \exists v \prec y\left(|v|=|z|+1 \wedge \bigwedge_{b \in \Sigma} L_{b}(z) \leftrightarrow L_{b}(v)\right),
\end{aligned}
$$

where $|v|=|u|+1$ is defined by $\exists w(w \lessdot u \wedge \operatorname{el}(w, v))$, and $w \lessdot u \equiv w \prec u \wedge \neg \exists t(w \prec t \wedge t \prec u)$. 
Strings as structures We shall use classical results on definability of strings represented as finite first-order structures. If $\Sigma=\left\{a_{1}, \ldots, a_{n}\right\}$, then a string $s \in \Sigma^{*}$ can be represented as a structure $M_{s}$ in the signature $\left(P_{a_{1}}, \ldots, P_{a_{n}},<\right)$. If $|s|=k$, then the universe of $M_{s}$ is $\{1, \ldots, k\},<$ is interpreted as the usual ordering, and $P_{a_{j}}$ is the set $\left\{i \mid 1 \leq i \leq k\right.$, and the $i$ th position of $s$ is $\left.a_{j}\right\}$.

If $\Phi$ is a sentence of some logic, it defines a language $L(\Phi)=\left\{s \in \Sigma^{*} \mid M_{s} \models \Phi\right\}$. When the logic is MSO, monadic second-order logic, the languages that arise this way are precisely the regular languages [20]. When the logic is FO, first-order, then the languages that arise are precisely the star-free languages (that is, those that can be obtained from $\emptyset$ and $\left\{a_{i}\right\}, i \leq n$ by using the operations of union, complement, and concatenation) [54].

Databases and query languages $\mathrm{A}$ database schema $S C$ is a collection of relation names $R_{1}, \ldots, R_{l}, R_{i}$ being of arity $p_{i}>0$. In an instance of $S C$ over a set $U$, each $R_{i}$ is interpreted as a finite subset of $U^{p_{i}}$. The active domain of a database $D, \operatorname{adom}(D)$, is the set of elements from $U$ that appear in $D$.

The general setting for query languages is that of a finite database and an infinite underlying structure $\mathcal{M}=\langle U, \Omega\rangle$, where $\Omega$ is a set of operations (functions and predicates) on $U$. As our basic language we consider relational calculus, or first-order logic, over the schema $S C$ and $\mathcal{M}$, denoted by $\operatorname{RC}(S C, \mathcal{M})$. We often omit $S C$ when it is understood, or irrelevant. Here we will focus exclusively on the string datatype, hence we will always have $U=\Sigma^{*}$. For example, if $\mathcal{M}=\left\langle\Sigma^{*}, \prec,\left(L_{a}\right)_{a \in \Sigma}\right\rangle$, the query

$$
\exists x R(x) \wedge L_{0}(x) \wedge \exists y\left(y \prec x \wedge L_{1}(y) \wedge(\neg \exists z y \prec z \prec x)\right)
$$

tests if there is a string in the relation $R$ which ends with 10. Indeed, it asks if the last symbol of $x$ is 0 , and if there exists a prefix $y$, which is the largest proper prefix of $x$ (as there is no $z$ with $y \prec z \prec x$ ) such that the last symbol of $y$ is 1 .

Given a query $\varphi\left(x_{1}, \ldots, x_{n}\right)$ in $\operatorname{RC}(S C, \mathcal{M})$ and $\vec{a} \in U^{n}$, we write $D \models \varphi(\vec{a})$ when $\varphi(\vec{a})$ is true in $(D, \mathcal{M})$. We write $\varphi(D)$ for the output of $\varphi$ on $D$, that is, $\left\{\vec{a} \in U^{n} \mid D \models \varphi(\vec{a})\right\}$. We say that $\varphi$ is safe on $D$ if $\varphi(D)$ is finite, and that $\varphi$ is safe if it is safe on every $D$. The safety problem is to determine whether a query is safe, and it is known to be undecidable even for the pure relational calculus [1]. The state-safety problem is to decide, for a given $\varphi$ and $D$, if $\varphi$ is safe on $D$.

We say that safe queries in $\mathrm{RC}(\mathcal{M})$ have effective syntax if there exists a recursively enumerable set $A$, of safe queries in $\mathrm{RC}(\mathcal{M})$ such that, for every $S C$, every safe $\operatorname{RC}(S C, \mathcal{M})$ query is equivalent to one in $A$.

Effective syntax is a first step towards an algebraic language expressing all safe queries. Indeed if such a language exists, safe queries must have effective syntax.

That effective syntax exists for safe queries in the pure relational calculus is a classical relational theory result [1]. Other results - both positive or negative - have been proved recently [11, 64].

Collapse results These establish very strong expressivity bounds for relational calculi. To formulate them, we need an important restriction of queries: to quantification over the active domain. We use quantifiers $\exists x \in a d o m$ and $\forall x \in$ adom, whose meaning is as follows: $D \models \exists x \in \operatorname{adom} \varphi(x, \cdot)$ if $D \models \varphi(a, \cdot)$ for some $a \in \operatorname{adom}(D)$ (as opposed to for some $a \in U$ in the case of the usual $\exists x$ quantifier), and similarly for the universal quantifier. These restricted quantifiers are definable in relational calculus, but it is often helpful to have them available separately.

A relational calculus formula is called an active-domain formula if all quantifiers in it are of the form $\forall x \in$ adom, $\exists x \in$ adom. We say that $\mathrm{RC}(\mathcal{M})$ admits natural-active collapse [10] if every $\mathrm{RC}(\mathcal{M})$ formula is equivalent to an active-domain formula. We say that $\operatorname{RC}(\mathcal{M})$ admits restricted quantifier collapse if every $\operatorname{RC}(\mathcal{M})$ formula is equivalent to one in which $S C$-relations appear only under the scope of quantifiers $\exists x \in a d o m$ and $\forall x \in a d o m$. Note that if $\mathcal{M}$ admits quantifier-elimination, these two notions coincide.

A query is generic if it commutes with permutations on the domain. The active-generic collapse [10] states that if an $\mathrm{RC}(\mathcal{M})$ formula with quantification of the form $\exists x \in$ adom and $\forall x \in$ adom expresses a generic query $Q$, then $Q$ must be expressible using only a linear order on the active domain, and no other predicates and functions from $\mathcal{M}$.

Model theory background Let $\Omega$ be a finite or countably infinite first-order signature, and $M$ a model over $\Omega$. By $\mathrm{FO}(M)$ we denote the set of all first-order formulae in the language of $\Omega$. The (complete) theory of $M, \mathrm{Th}(M)$, is 
the set of all sentences in $\mathrm{FO}(M)$ true in $M$. Two models $M$ and $M^{\prime}$ over $\Omega$ are elementary equivalent if $\operatorname{Th}(M)=$ $\operatorname{Th}\left(M^{\prime}\right)$.

We say that $M$ admits quantifier elimination $(Q E)$ if for every formula $\varphi(\vec{x})$ in $\operatorname{FO}(M)$ there is a quantifier-free formula $\varphi^{\prime}(\vec{x})$ such that $\forall \vec{x} \varphi(\vec{x}) \leftrightarrow \varphi^{\prime}(\vec{x})$ is true in $M$. In every case where we show quantifier-elimination for a model in this paper, the conversion to a quantifier-free formula can be made effective, although in several cases (e.g. Theorem 3.12) we will not give the details of the effective versions.

For a tuple $\vec{a}$ and a model $M$ over $\Omega$, we let $t p_{M}(\vec{a})$ be the type of $\vec{a}$ in $M$ (the set of all formulae of $\operatorname{FO}(M)$ satisfied by $\vec{a}$ ), and $\operatorname{atp}_{M}(\vec{a})$ be the atomic type in $M$ (the set of all quantifier-free formulae of $\mathrm{FO}(M)$ satisfied by $\vec{a}$ ). If $A$ is a subset of $M, t p_{M}(\vec{a} / A)$ is the type of $\vec{a}$ over $A$ in $M$ (the set of all FO-formulae over $\Omega \cup A$ satisfied by $\vec{a}$ ).

An $\omega$-saturated model $M$ over $\Omega$ is a model such that each consistent type (a type is consistent if it has a witness in at least one model of $\Omega$ ) over a finite set $A$ in $\mathrm{FO}(M)$ is satisfied in $M$. It is known [21] that every model $M$ over $\Omega$ has an elementary equivalent $\omega$-saturated model $M^{*}$.

Many proofs use Ehrenfeucht-Fraïssé games [28, 33, 27]. For two structures $\mathcal{M}_{1}$ and $\mathcal{M}_{2}$ of the same vocabulary, we write $\mathcal{M}_{1} \equiv_{k} \mathcal{M}_{2}$ if the duplicator has a winning strategy in the $k$-round game on $\mathcal{M}_{1}$ and $\mathcal{M}_{2}$ (that is, if $\mathcal{M}_{1}$ and $\mathcal{M}_{2}$ agree on all sentences of quantifier rank up to $k$ ). We also assume familiarity with Monadic Second Order Logic (MSO) [27]. Some proofs will use MSO games [27]; we write $\mathcal{M}_{1} \equiv_{\mathrm{MSO}_{k}} \mathcal{M}_{2}$ if the duplicator has a winning strategy in the $k$-round MSO game, which similarly means the two structures can not be distinguished by MSO-sentences of quantifier depth $k$.

Isolation, VC-dimension, and collapse We review several model-theoretic concepts that prove useful in establishing bounds on the expressive power of query languages.

Let $T$ be a theory over $\Omega$ and $M$ be a model of $T$. A subset $A$ of $M$ is said to be pseudo-finite if $(M, A) \models F(T, P)$, where $P$ is a unary predicate, and $F(T, P)$ is the set of all formulae of $\operatorname{FO}(\Omega \cup\{P\})$ satisfied by all finite sets of elements in any model of $T$.

If $p$ is a type over $A$ in $M$, a subset $q$ of $p$ isolates $p$ if $p$ is the only type over $A$ in $M$ containing $q$. A complete theory $T$ over $\Omega$ is said to have the strong isolation property if for any model $M$ of $T$ and any pseudo-finite set $A$ and any element $a$ in $M$, there is a finite subset $A_{0}$ of $A$ such that $t p_{M}\left(a / A_{0}\right)$ isolates $t p_{M}(a / A)$. We say that it has the isolation property if a countable $A_{0}$ exists as above.

Isolation is an interesting property in the database context because it implies the restricted quantifier collapse $[8,32]$. Here we also use it to provide bounds on the VC-dimension of definable families.

For a family $\mathcal{C}$ of subsets of a set $U$, and a set $F \subseteq U$, we say that $\mathcal{C}$ shatters $F$ if $\{F \cap C \mid C \in \mathcal{C}\}$ is the powerset of $F$. The $V C$-dimension of $\mathcal{C}$ is the maximum cardinality of a finite set shattered by $\mathcal{C}$ (or $\infty$, if arbitrarily large finite sets are shattered by $\mathcal{C}$ ). This concept is fundamental to learning theory, as finite VC-dimension of a hypothesis space is equivalent to learnability (PAC-learnability) $[5,15]$.

Now consider a structure $M=\left\langle\Sigma^{*}, \Omega\right\rangle$, and a $\mathrm{FO}(M)$ formula $\varphi(\vec{x}, \vec{y})$. For each $\vec{a}$, let $\varphi(\vec{a}, M)=\{\vec{b} \mid M \models$ $\varphi(\vec{a}, \vec{b})\}$. The family of sets $\varphi(\vec{a}, M)$, where $\vec{a}$ ranges over all tuples over $M$, is called a definable family. We say that $M$ has finite VC-dimension if every definable family has finite VC-dimension. In particular, this implies learnability of FO-definable families over $M$.

We shall see more connections between isolation, $\mathrm{VC}$ dimension, and collapse results later in the paper.

Complexity classes Some complexity results in this paper refer to parallel complexity classes $\mathrm{AC}^{0}, \mathrm{TC}^{0}$, and $\mathrm{NC}^{1}$. $\mathrm{AC}^{0}$ is constant parallel time; more precisely, the class of languages accepted by polynomial-size constant-depth unbounded fan-in circuits. $\mathrm{TC}^{0}$ additionally has majority gates of unbounded fan-in. $\mathrm{In} \mathrm{NC}^{1}$, there are no majority gates, the depth is allowed to be logarithmic, but fan-in is bounded. It is known that $\mathrm{AC}^{0} \subset \mathrm{TC}^{0} \subseteq \mathrm{NC}^{1}$ (parity separates $\mathrm{TC}^{0}$ from $\mathrm{AC}^{0}$ ). We consider uniform versions of these classes [7]; uniform $\mathrm{AC}^{0}$ over finite structures can be characterized via definability in $\mathrm{FO}(\mathrm{BIT},<)$ : first-order logic with linear order and the BIT predicate $(\mathrm{BIT}(i, j)$ is true iff the $j$ th bit in the binary representation of $i$ is one.) To capture uniform $\mathrm{TC}^{0}$ it suffices to add counting quantifiers to $\mathrm{FO}(\mathrm{BIT},<)[7]$.

PH is the polynomial hierarchy, which contains, e.g., NP and coNP and is itself included in PSPACE [57].

As usual, for data complexity, one fixes a query $Q$ and considers the complexity of $\{\operatorname{enc}(D) \# \operatorname{enc}(t) \mid t \in Q(D)\}$, where enc is an encoding of databases and tuples over some fixed alphabet, typically $\{0,1\}$ [1]. Normally in pure 
relational calculus the encoding is such that the active domain is considered to be $\{1, \ldots, k\}$, and each number $i$ is represented in binary. When we deal with interpreted elements stored in a database, such an encoding is not appropriate, as one needs to take into account operations on those interpreted elements. In particular, in the case of strings over a finite alphabet, we consider the encoding of a string to be itself (in the case of an alphabet different from $\{0,1\}$ we may have to code letters in $\{0,1\}$ first).

\section{Model theory of strings}

In this section we study logical definability over $\mathbf{S}_{\text {len }}, \mathbf{S}, \mathbf{S}_{\text {left }}$ and two other structures, defining regular algebras over $\Sigma^{*}$. We are particularly interested in quantifier-elimination results, and in some model-theoretic properties (isolation, $\mathrm{VC}$ dimension) that will later give us results about the expressive power of the relational calculi based on these structures. We start with the strongest regular algebra $\mathbf{S}_{\text {len }}$, then move to the star-free algebra $\mathbf{S}$, and to a more expressive star-free algebra $\mathbf{S}_{\text {left }}$. The quantifier-elimination proof for the latter is technically the most involved result in this section. We then show how to expand $\mathbf{S}$ and $\mathbf{S}_{\text {left }}$ to regular algebras, without losing their nice properties.

\subsection{A regular algebra based on $\mathrm{S}_{\text {len }}$}

In this subsection we will focus on the structure $\mathbf{S}_{\text {len }}$. We will assume here that the alphabet $\Sigma$ contains at least two letters. For a 1-letter alphabet, it is easy to see that $\mathbf{S}_{\text {len }}$ reduces to $\mathbf{S}$, which will be dealt with in the next subsection.

\subsubsection{Automata and Definability}

A letter-by-letter automaton is a usual DFA whose alphabet is $(\Sigma \cup\{\#\})^{n}, \# \notin \Sigma$. An $n$-tuple of strings $s_{1}, \ldots, s_{n}$ can be viewed as a word of length $\max _{i}\left|s_{i}\right|$ over the alphabet $\Sigma \cup\{\#\}$, where the $j$ th letter is the tuple $\left(s_{1}^{j}, \ldots, s_{n}^{j}\right)$; here $s_{k}^{j}$ is the $j$ th letter of $s_{k}$, if $\left|s_{k}\right| \leq j$, and \# otherwise. We say that a predicate $P \subseteq\left(\Sigma^{*}\right)^{n}$ is definable by a letter-to-letter $n$-automaton $A$ if $\left(s_{1}, \ldots, s_{n}\right) \in P$ iff $A$ accepts $s_{1}, \ldots, s_{n}$.

As mentioned in the introduction, $\mathbf{S}_{\text {len }}=\left\langle\Sigma^{*}, \preceq,\left(l_{a}\right)_{a \in \Sigma}\right.$, el $\rangle$ is the canonical automatic structure, and relations definable in $\mathbf{S}_{\text {len }}$ are precisely the regular relations, that is, $k$-ary definable relations are precisely those given by letterto-letter $k$-automata $[14,19]$. In particular, this gives a normal form for $\mathbf{S}_{\text {len }}$-formulae. We introduce a new type of length-bounded quantifiers of the form $\exists|x| \leq|y|$ and $\forall|x| \leq|y|$. A formula $\exists|x| \leq|y| \varphi$ is meant as an abbreviation for $\exists x((|x| \leq|y|) \wedge \varphi)$.

Since every finite automaton can be simulated by a length-bounded $\mathrm{FO}\left(\mathbf{S}_{\text {len }}\right)$ formula, we conclude that each $\mathrm{FO}\left(\mathbf{S}_{\text {len }}\right)$ formula is equivalent to a length-bounded $\mathrm{FO}\left(\mathbf{S}_{\text {len }}\right)$ formula. Note that this result can also be shown directly by an Ehrenfeucht-Fraïssé game argument.

\subsubsection{Quantifier Elimination}

The universal property of $\mathbf{S}_{\text {len }}$ mentioned above indicates that $\mathbf{S}_{\text {len }}$ may be "too rich" in relations for many applications. We present evidence for this by addressing the open question of $[22,55]$ whether $\mathbf{S}_{\text {len }}$ has quantifier elimination in a reasonable signature. One first needs to define what "reasonable" means here. Clearly, every structure has quantifier elimination in a sufficiently large expansion of the signature: add symbols for all definable predicates, for example. One can thus take reasonable to mean a finite expansion, but this is not satisfactory: for example, Presburger arithmetic has quantifier elimination in an infinite signature $\left(+,<, 0,1,(\bmod k)_{k>1}\right)$ [31]. Note however that in this example, the maximum arity of the predicates and functions is 2 . In fact, it appears to be a common phenomenon that when one proves quantifier elimination in an infinite signature, there is an upper bound on the arity of functions and predicates in it.

We thus view this condition as necessary for a signature to be "reasonable". In general, a reasonable signature might contain relation symbols as well as function symbols. Nevertheless, we can rule out the possibility of a signature with function symbols of arity at most 1 for which $\mathbf{S}_{\text {len }}$ has quantifier elimination. This is in contrast to the weaker structures that we consider, all of which have quantifier elimination in a relational signature of bounded arity. Let 
$\mathbf{S}_{\text {len }}^{(n, m)}$ be the expansion of $\mathbf{S}_{\text {len }}$ with all definable predicates of arity at most $n$, and definable functions of arity at most $m$. We show the following:

Theorem 3.1 (a) For any $n \geq 0$, and $m=0,1, \mathbf{S}_{\text {len }}^{(n, m)}$ does not have $Q E$.

(b) $\mathbf{S}_{\mathrm{len}}^{(1,2)}$, the expansion of $\mathbf{S}_{\mathrm{len}}$ with all unary predicates and binary functions, has $Q E$.

Proof. (a). We assume $\Sigma=\{0,1\}$ and fix $n$. Let $m=0$. The definable property which can not be expressed by a quantifier-free formula is defined as follows. It holds for a tuple $x_{1}, \ldots, x_{n+1}$ of strings, if there is a position $i$ such that the $i$ th symbol in all $x_{j} \mathrm{~s}$ is 0 .

This is clearly definable in $\mathbf{S}_{\text {len }}$ by $\varphi\left(x_{1}, \ldots, x_{n+1}\right)$ :

$$
\exists y_{1}, \ldots, y_{n+1} \bigwedge_{j} y_{j} \preceq x_{j} \wedge \bigwedge_{j} L_{0}\left(y_{j}\right) \wedge \bigwedge_{j, k} \operatorname{el}\left(y_{j}, y_{k}\right)
$$

We now assume that $\varphi$ is a Boolean combination of formulae depending on $n$ variables each. Let these formulae be named as $\alpha_{j}^{i}, i \in\{1, \ldots, n+1\}, j \in\left\{1, \ldots, l_{i}\right\}$, where $\alpha_{j}^{i}$ does not have $x_{i}$ as free variable.

By [14], each $\alpha_{j}^{i}$ is given by a letter-to-letter $n$-automaton $A_{j}^{i}$ over $\Sigma^{n}$. Let $m$ be the maximum number of states of the $A_{j}^{i}$.

Now let $p_{1}<p_{2}<\ldots<p_{n+1}$ be primes with $p_{1}>m+1$. Let $\pi_{i}=\prod_{j \neq i} p_{j}$, and let $P=\prod_{j} p_{j}\left(=\pi_{i} \cdot p_{i}\right.$, for each $i$ ).

We now define $\omega$-words $w^{j}, j=1, \ldots, n+1$, by

$$
w^{j}[k]= \begin{cases}0 & k=0\left(\bmod p_{j}\right) \\ 1 & \text { otherwise }\end{cases}
$$

where, as for finite strings, $w^{j}[k]$ denotes the $k$ th position in $w^{j}$.

Now fix $i \leq n+1$ and $s \leq l_{i}$, and consider a run of $A_{s}^{i}$ on $\left(w^{j}, j \neq i\right)$ (that is, the $k$ th input symbol is $\left.\left(w^{1}[k], \ldots, w^{i-1}[k], w^{i+1}[k], \ldots, w^{n+1}[k]\right)\right)$. At every position that is equal to 0 modulo $\pi_{i}$ (and only at those positions), the input symbol is $\overrightarrow{0}=(0, \ldots, 0)$. Moreover, for any $l \geq 0$ and any $c_{1}, c_{2}>0$, the input symbols are the same at positions $l+c_{1} \cdot \pi_{i}$ and $l+c_{2} \cdot \pi_{i}$.

We now consider positions equal to 0 modulo $\pi_{i}$; since $A_{s}^{i}$ has at most $m$ states, we can find two numbers $d_{1}<$ $d_{2} \leq m+1$ (depending on $s$ ) such that in positions $d_{1} \cdot \pi_{i}$ and $d_{2} \cdot \pi_{i}$ the automaton $A_{s}^{i}$ is in the same state $q$, reading $\overrightarrow{0}$. Let $d=\left(d_{2}-d_{1}\right) \cdot \pi_{1}$. Thus, at every position $d_{1} \cdot \pi_{i}+k \cdot d$, the automaton is in the state $q$, reading $\overrightarrow{0}$.

Then for every $l \geq 0$ and every $k \geq 0$, we have that $A_{s}^{i}$ is in the same state in positions $d_{1} \cdot \pi_{i}+l$ and $d_{1} \cdot \pi_{i}+l+k \cdot d$, and reads the same symbol in those states. Furthermore, notice that $d_{2} \cdot \pi_{i} \leq(m+1) \cdot \pi_{i}<p_{1} \cdot \pi_{i} \leq p_{i} \cdot \pi_{i}=P$.

Summing up, for each $A_{s}^{i}$, we have two constants, $a_{s}^{i}\left(=d_{1} \cdot \pi_{i}\right)$ and $b_{s}^{i}(=d)$, such that $a_{s}^{i}<P$ and the state of $A_{s}^{i}$ is the same in positions $a_{s}^{i}+l$ and $a_{s}^{i}+l+k \cdot b_{s}^{i}$, for $l, k \geq 0$.

Now let $C=\max _{i, s} a_{s}^{i}$ and $C^{\prime}=C+P \cdot \prod_{i, s} b_{s}^{i}$. We have $C^{\prime}>P>C$, and all automata $A_{s}^{i}$ are in the same state in positions $C$ and $C^{\prime}$. In particular, if $w^{j}[1, k]$ denotes the finite word that consists of the first $k$ positions of $w^{j}$, we have that every $\alpha_{j}^{i}$ agrees on

$$
\left(w^{1}[1, C], \ldots, w^{i-1}[1, C], w^{i+1}[1, C], \ldots, w^{n+1}[1, C]\right)
$$

and

$$
\left(w^{1}\left[1, C^{\prime}\right], \ldots, w^{i-1}\left[1, C^{\prime}\right], w^{i+1}\left[1, C^{\prime}\right], \ldots, w^{n+1}\left[1, C^{\prime}\right]\right)
$$

The assumption that $\varphi$ is a Boolean combination of $\alpha_{j}^{i}$ s now gives us that $\varphi$ agrees on $\left(w^{1}[1, C], \ldots, w^{n+1}[1, C]\right)$ and $\left(w^{1}\left[1, C^{\prime}\right] \ldots, w^{n+1}\left[1, C^{\prime}\right]\right)$, which is impossible, since $\varphi\left(w^{1}[1, C], \ldots, w^{n+1}[1, C]\right)$ is false $(C<P$ and there is no position with all zeros in it) and $\varphi\left(w^{1}\left[1, C^{\prime}\right], \ldots, w^{n+1}\left[1, C^{\prime}\right]\right)$ is true $\left(C^{\prime}>P\right.$, and in position $P$ all symbols are 0$)$.

For the case of $m=1$, it suffices to notice that for any $n>1$, any quantifier-free formula $\alpha\left(x_{1}, \ldots, x_{n}\right)$ in $\mathbf{S}_{\text {len }}^{(n, 1)}$ is equivalent to a quantifier-free formula in $\mathbf{S}_{\text {len }}^{(n, 0)}$. For instance $R(f(x), f(y))$ where $R$ is a definable $\mathbf{S}_{\text {len }}^{(2,0)}$ relation, is equivalent to $R_{f, g}(x, y)$, where $R_{f, g}$ is the $\mathbf{S}_{\text {len }}^{(2,0)}$ relation defined by $R(f(x), f(y))$. 
Proof of (b).

Let us assume that $\Sigma$ contains at least the symbols 0 and 1 and let $\mathbf{S}_{\text {len }}^{+}$be the expansion of $\mathbf{S}_{\text {len }}$ by the following definable functions and predicates:

- the binary functions $f_{\wedge}, f_{\vee}$ which are the bitwise AND and OR of two 0-1 strings $u$ and $v$, respectively (and $\epsilon$ for non-0-1-inputs). When $u$ and $v$ do not have the same length we add sufficiently many 0 s to the right of the shorter string. Thus the length of the result is $\max (|u|,|v|)$. E.g., $f_{\wedge}(101,11)=100$;

- the unary function $f_{\neg}$ which is the bitwise NOT of a 0-1 string;

- for each $\sigma \in \Sigma$, a unary function $\operatorname{Fil}_{\sigma}$, where $\operatorname{Fil}_{\sigma}(w)$ has a 1 at position $i$ iff $w[i]=\sigma$ and a 0 otherwise;

- for each $j, k, j<k$, a unary function $\operatorname{Pat}_{j, k}$ where $\operatorname{Pat}_{j, k}(w)$ has the same length as $w$ and has a 1 at position $i$ iff $i \equiv j(\bmod k)$ and a 0 otherwise;

- unary functions LShift, RShift, where RShift $(w)$ is obtained from $w$ by deleting the last (rightmost) symbol and LShift $(w)$ is obtained from $w$ by deleting the first (leftmost) symbol;

- for each $j, m, j<m$, the unary predicate $P_{m, j}$ which will be defined below.

Let $R$ be an $n$-ary relation over $\Sigma$, definable in $\mathbf{S}_{\text {len }}$. Our goal is to find a quantifier-free $\mathbf{S}_{\text {len }}^{+}$-formula $\varphi$ such that, for each $n$-tuple $\vec{w}$ of strings, $\mathbf{S}_{\text {len }}^{+} \models \varphi(\vec{w})$ iff $\vec{w} \in R$.

We know from $[14,19]$ that the relations definable in $\mathbf{S}_{\text {len }}$ are precisely the regular relations, that is, precisely those given by letter-to-letter $n$-automata $[14,19]$.

Let $A$ be such an automaton for $R$ over the alphabet $(\Sigma \cup\{\#\})^{n}$ with state set $Q_{m}=\left\{q_{0}, \ldots, q_{m-1}\right\}$, initial state $q_{0}$, transition function $\delta$ and set $F$ of accepting states.

An $m$-state behavior function is any function $f: Q_{m} \rightarrow Q_{m}$. An $m$-state behavior function can be encoded into a binary behavior string $b(f)$ of length $M:=m^{2}$ as follows. For $j, j^{\prime}<m$, position $j m+j^{\prime}+1$ of $b(f)$ is 1 iff $f\left(q_{j}\right)=q_{j^{\prime}}$.

Let $P_{m, j}, j<m$, be the unary predicate which holds for all strings $u=b_{1} \cdots b_{l}$, where each $b_{i}$ encodes an $m$-state behavior function $f^{i}$ and $f^{l}\left(\cdots\left(f^{1}\left(q_{0}\right)\right) \cdots\right)=q_{j}$. As the blocks $b_{i}$ are of constant length these predicates are regular.

The idea of the proof is to map each block of the input of length $m^{2}$ to the string which describes the behavior of $A$ on this block. Whether $A$ accepts the input can then be expressed by means of the predicates $P_{m, j}$.

For a given $n$-tuple $\vec{w}$, let $l$ be minimal such that $l M \geq|\vec{w}|$ where $|\vec{w}|=\max \left(\left|w_{1}\right|, \ldots,\left|w_{n}\right|\right)$ and let $f_{\vec{w}}^{i}=$ $\delta^{*}(\cdot, \vec{w}[(i-1) M+1, i M])$, for $i<l$ and $f_{\vec{w}}^{l}=\delta^{*}(\cdot, \vec{w}[(l-1) M+1,|\vec{w}|])$. Then the state of $A$ after reading $\vec{w}$, starting from the initial state $q_{0}$, is $j$ if and only if $b\left(f_{\vec{w}}^{1}\right) \cdots b\left(f_{\vec{w}}^{l}\right) \in P_{m, j}$.

Hence, it is sufficient to find an $\mathbf{S}_{\text {len }}^{+}$-term $\theta$ such that $\theta(\vec{w})=b\left(f_{\vec{w}}^{1}\right) \cdots b\left(f_{\vec{w}}^{l}\right)$. The construction of $\theta$ is described in two steps.

First, let $f_{\max }(\vec{w})$ be defined as $\bigvee_{\sigma \in \Sigma} \bigvee_{i=1}^{n} \operatorname{Fil}_{\sigma}\left(w_{i}\right)$. Here, as in the following the Boolean operators are abbreviations for the respective terms using $f_{\vee}, f_{\wedge}, f_{\neg}$. Note that $f_{\max }(\vec{w})$ defines a string of length $\max \left\{\left|w_{i}\right| \mid\right.$ $i \leq n\}$ consisting only of ones. Further let $\widehat{\operatorname{Fil}}_{\sigma, i}(\vec{w})$ be the term $f_{\max }(\vec{w}) \wedge \operatorname{Fil}_{\sigma}\left(w_{i}\right)$ and let $\widehat{\operatorname{Fil}} \#, i(\vec{w})$ be $f_{\max }(\vec{w}) \wedge \neg\left(\bigvee_{\sigma \in \Sigma} \operatorname{Fil}_{\sigma}\left(w_{i}\right)\right)$. Hence, for each symbol $\tau \in \Sigma \cup\{\#\}, \widehat{\mathrm{Fil}}_{\tau, i}(\vec{w})$ has a 1 at position $j$, if the automaton $A$ reads a $\tau$ as the $j$-th symbol of $w_{i}$.

Now we are ready to finish the description of $\theta$. For simplicity, we describe $\theta$ for the case where $\left|f_{\max }(\vec{w})\right|$ is a multiple of $M$. The general case is slightly more complicated. $\theta(\vec{w})$ has to carry a 1 at a position $\left(j_{0}-1\right) M+j m+$ $j^{\prime}+1$, for $j, j^{\prime}<m, j_{0}>0$, iff the tuple $\vec{w}\left[\left(j_{0}-1\right) M+1, j_{0} M\right]$, consisting of $n$ strings of length $M$ is in the set $T\left(j, j^{\prime}\right):=\left\{\vec{s} \mid \delta^{*}(j, \vec{s})=j^{\prime}\right\}$. Therefore $\theta$ can be expressed as

$$
\bigvee_{j, j^{\prime}} \bigvee_{\vec{s} \in T\left(j, j^{\prime}\right)}\left[\operatorname{Pat}_{l, M}\left(f_{\max }(\vec{w})\right) \wedge \bigwedge_{i=1}^{l} \bigwedge_{k=1}^{n} \operatorname{RShift}^{(l-i)}\left(\widehat{\operatorname{Fil}}_{s_{k}[i], k}(\vec{w})\right) \wedge \bigwedge_{i=l+1}^{M} \bigwedge_{k=1}^{n} \operatorname{LShift}^{(i-l)}\left(\widehat{\operatorname{Fil}}_{s_{k}[i], k}(\vec{w})\right)\right]
$$

where $l$ is a shorthand for $j m+j^{\prime}+1$ and $f^{(i)}$ denotes the $i$-fold application of $f$. 
The formula says the following: Assume $0<l \leq M$ and $\vec{s} \in T\left(j, j^{\prime}\right)$ fixed, a block $\vec{w}\left[\left(j_{0}-1\right) M+1, j_{0} M\right]$ of size $M$ is viewed centered in its $l$ th position and thus has $l-1$ characters on its left and $M-l$ on its right. The last part of the formula checks for the blocks of size $M$ centered in $l$ that equal $\vec{s}$. The test is made separately for the left and right part (this corresponds to the variable $i$ ) and for each element of $\vec{s}$ (this corresponds to the variable $k$ ). All the results of the tests are shifted to the right for the left part of the block and to the left for the right part in order to align them on the centered position $l$. Thus the big bitwise $\bigwedge$ is true iff all the previous tests were true and thus iff the block of size $M$ centered in $l$ equals $\vec{s}$.

The first part of the formula filters the blocks we are interested in by keeping only the one centered in $j m+j^{\prime}+1$ modulo $M$. The second bitwise $\bigvee$ will check for all possibilities for $\vec{s} \in T\left(j, j^{\prime}\right)$ thus the $j m+j^{\prime}+1$ modulo $M$ positions will be equal to 1 iff the corresponding block is a string of $T\left(j, j^{\prime}\right)$ as desired. The first bitwise $\bigvee$ ensures that we cover all positions.

\subsubsection{VC-Dimension}

Our next result shows another model-theoretic and learning-theoretic shortcoming of $\mathbf{S}_{\text {len }}$ : namely, a single formula $\varphi(x, y)$ can define a widely varying collection of relations as we let the parameter $x$ vary. We formalize this through the notion of VC-dimension.

Proposition 3.2 There are definable families in $\mathbf{S}_{\mathrm{len}}$ that have infinite $V C$-dimension.

Proof. Let $\Sigma=\{0,1\}$, and let $\varphi(x, y)$ be $\exists z\left(z \prec x \wedge \operatorname{el}(z, y) \wedge L_{1}(z)\right)$. Let $\mathcal{C}$ be the corresponding definable family: $S \in \mathcal{C}$ iff $S=\varphi\left(s, \mathbf{S}_{\text {len }}\right)$ for some string $s$. Let $A_{n}=\left\{0^{i} \mid i<n\right\}$. Then $A_{n}$ is shattered by $\mathcal{C}$ : given any subset $X$ of $A_{n}$, let $s_{X}$ be a string of length $n$ where the $i$ th character is 1 iff $0^{i} \in X$. Then $\varphi\left(s_{X}, \mathbf{S}_{\text {len }}\right) \cap A_{n}=X$. Since $n$ was arbitrary, this shows that $\mathcal{C}$ has infinite VC-dimension.

\subsection{A star-free algebra based on $\mathrm{S}$}

We now turn to the most obvious analog of $\mathbf{S}_{\text {len }}$ for the star-free sets. This is the model $\mathbf{S}=\left\langle\Sigma^{*}, \preceq,\left(l_{a}\right)_{a \in \Sigma}\right\rangle$, which is the most basic model among those studied in the paper. We show that it has remarkably nice behavior: it admits effective QE in a rather small extension to the signature. This immediately tells us that the definable subsets of $\Sigma^{*}$ are precisely the star-free languages. We then characterize the $n$-dimensional definable relations in $\mathbf{S}$ by their closure properties, and by an automaton model.

Note that $\mathbf{S}$ is very close to strings considered as term algebras, that is, to $\left\langle\Sigma, \epsilon,\left(l_{a}\right)_{a \in \Sigma}\right\rangle$. It is well-known that the theory of arbitrary term algebras is decidable and admits QE [53, 44]. However, adding the prefix relation is not necessarily a trivial addition: for arbitrary term algebras with prefix (subterm), only the existential theory is decidable, but the full theory is undecidable [68] (similar results hold for other orderings on terms [23]). The undecidability result of [68] requires at least one binary term constructor; our results indicate that in the simpler case of strings one recovers $\mathrm{QE}$ with the prefix relation.

\subsubsection{A Normal Form for $\mathrm{S}$}

We start with a result that gives a normal form for formulae of $\mathrm{FO}(\mathbf{S})$.

For that, we need the following predicates, introduced in [52]. For each $L \subseteq \Sigma^{*}$, let $P_{L}$ be the set of pairs $(x, y)$ of strings such that $x \preceq y$ and $y-x \in L$. The following lemma is obvious, since it is well-known that star-free sets are first-order definable on string models [54].

Lemma 3.3 For each star free language $L$, there is a formula $\varphi_{L}(x, y)$ in $\mathrm{FO}(\mathbf{S})$ which defines $P_{L}$.

We now give a normal form result for $\mathrm{FO}(\mathbf{S})$.

Proposition 3.4 Every formula $\psi(\vec{x})$ in $\mathrm{FO}(\mathbf{S})$ can be effectively transformed into an equivalent formula which is a disjunction of formulae of the form

$$
\gamma(\vec{x}) \wedge \delta(\vec{x})
$$


where $\gamma(\vec{x})$ is a complete tree-order description over $\vec{x}$ and $\delta(\vec{x})$ is a conjunction of formulae of the form $\varphi_{L}\left(t(\vec{x}), t^{\prime}(\vec{x})\right)$, where $L$ is star-free, each of $t(\vec{x})$ and $t^{\prime}(\vec{x})$ is either $\epsilon$ or a term of the form $x_{i} \sqcap x_{j}$, and $\gamma(\vec{x})$ implies that $t^{\prime}(\vec{x})$ is an immediate successor of $t(\vec{x})$ in the tree-order.

Proof. The proof is by induction on the structure of $\psi$. The base case of the induction is handled by noting that the atomic formulae are binary, and the basic formulae $x \prec y$ and and $y=x \cdot a$ are simple cases of $\varphi_{L}(x, y)$.

Note that for any conjunction $\chi(\vec{x})$ of formulae of the form $t_{1}(\vec{x})\{\prec,=\} t_{2}(\vec{x})$ and their negations (where $t_{1}, t_{2}$ are $\sqcap, \epsilon$-terms), there are finitely many complete tree order descriptions $\gamma_{i}, i \in I$ over $\vec{x}$ which are consistent with $\chi$, and furthermore, all such $\gamma_{i}$ 's can be effectively found. Thus, any conjunction of two formulae in the normal form, $\xi_{1}(\vec{x}) \wedge \xi_{2}(\vec{x})$, can be put in the form $\bigvee_{i \in I} \gamma_{i}(\vec{x}) \wedge \chi(\vec{x})$, where $\chi(\vec{x})$ is a conjunction of formulae $\varphi_{L}\left(t(\vec{x}), t^{\prime}(\vec{x})\right)$. This is almost in the normal form, but $\gamma_{i}$ may not imply that $t^{\prime}(\vec{x})$ is an immediate successor of $t(\vec{x})$ in the tree-order. If that is the case, choose some term $t^{\prime \prime}(\vec{x})$ such that $t(\vec{x}) \prec t^{\prime \prime}(\vec{x}) \prec t^{\prime}(\vec{x})$. By a decomposition argument similar to the one used in the proof of Theorem 4.4 in [67], there exists a finite sequence of pairs of star-free languages $\left(L_{j}^{\prime}, L_{j}^{\prime \prime}\right)$ such that $\varphi_{L}\left(t(\vec{x}), t^{\prime}(\vec{x})\right)$ is equivalent to $\bigvee_{j}\left(\varphi_{L_{j}^{\prime}}\left(t(\vec{x}), t^{\prime \prime}(\vec{x})\right) \wedge \varphi_{L_{j}^{\prime \prime}}\left(t^{\prime \prime}(\vec{x}), t^{\prime}(\vec{x})\right)\right)$. We can now propagate disjunction and repeat the process until for all formulae of the form $\varphi_{L}\left(t(\vec{x}), t^{\prime}(\vec{x})\right), \gamma_{i}$ implies that $t^{\prime}(\vec{x})$ is an immediate successor of $t(\vec{x})$. This shows that any Boolean combination of formulae in the normal form can be put in the normal form itself.

Thus, the only nontrivial case is $\psi=\exists x \rho(x, \vec{y})$. By induction, we can assume that $\rho$ is in the required form. So we have

$$
\psi=\exists x \bigvee_{i}\left(\gamma_{i}(x, \vec{y}) \wedge \bigwedge_{j} \delta_{i j}(x, \vec{y})\right)
$$

where the $\gamma_{i}$ are tree-order descriptions, and the $\left.\delta_{i j}(x, \vec{y})\right)$ are of the form $\varphi_{L}\left(t(x, \vec{y}), t^{\prime}(x, \vec{y})\right)$. Thus, it suffices to show how to eliminate $x$ from $\beta(\vec{y})=\exists x \gamma(x, \vec{y}) \wedge \bigwedge_{j} \varphi_{L_{j}}\left(t_{j}(x, \vec{y}), t_{j}^{\prime}(x, \vec{y})\right)$ where $\gamma$ is a complete tree-order description, all $L_{j}$ s are star-free, and each $t_{j}, t_{j}^{\prime}$ is a $\epsilon, \sqcap$-term, such that $\gamma$ implies that $t_{j}^{\prime}$ is an immediate successor of $t_{j}$ in the tree-order. We can further assume without loss of generality that for every pair of terms $t_{j}, t_{j}^{\prime}$, there is at most one formula of the form $\varphi_{L_{j}}\left(t_{j}, t_{j}^{\prime}\right)$ in the conjunction (if not, one can take the intersection of all the languages in such formulae for these two terms, which will still be star-free). Furthermore, assume $\gamma$ sets one of the $y_{l}$ to $\epsilon$ (if not, add an extra variable and set it to $\epsilon$ in $\gamma$ ). Let $\gamma^{\prime}(\vec{y})$ be the restriction of $\gamma$ to $\vec{y}$ (that is, complete tree-order description of $\operatorname{Tree}(\vec{y})$ implied by $\gamma$ ).

We now consider four cases, depending on the relationship between $x$ and Tree $(\vec{y})$ which is implied by $\gamma(x, \vec{y})$. First, assume that $\gamma(x, \vec{y})$ implies that $x$ is a node in $\operatorname{Tree}(\vec{y})$, that is, $\epsilon$ or $y_{i} \sqcap y_{j}$ for some $i, j$. In this case every term of the form $x \sqcap y_{k}$ can be rewritten as a term that only uses $\vec{y}$ variables, and every formula of the form $\varphi_{L_{j}}\left(t_{j}(x, \vec{y}), t_{j}^{\prime}(x, \vec{y})\right)$ is thus equivalent to a disjunction of formulas $\varphi_{L_{j}}\left(\tau_{j}(\vec{y}), \tau_{j}^{\prime}(\vec{y})\right)$, where $\tau_{j}, \tau_{j}^{\prime}$ are the result of eliminating $x$ from $t_{j}, t_{j}^{\prime}$. Thus, $\beta$ is equivalent to a disjunction of formulas of the form $\gamma^{\prime}(\vec{y}) \wedge \bigwedge_{j} \varphi_{L_{j}}\left(\tau_{j}(\vec{y}), \tau_{j}^{\prime}(\vec{y})\right)$.

In the second case, $\gamma(x, \vec{y})$ implies that $x$ is not a prefix of any $y_{k}$ from $\vec{y}$, and that the meet of $x$ and $\vec{y}$ is a node $y_{i} \sqcap y_{j}$ in Tree $(\vec{y})$. In this case we may have a formula of the form $\varphi_{L}\left(y_{i} \sqcap y_{j}, x\right)$ as a conjunct in $\beta$. The case is handled just as the previous one, except that we need to deal with the formula $\varphi_{L}\left(y_{i} \sqcap y_{j}, x\right)$ (which is the only formula in this case that mentions $x$ ). The existence of $x$ satisfying it is guaranteed iff there exists a string in $L$ with a first symbol $a$ such that $\left(y_{i} \sqcap y_{j}\right) \cdot a$ is not a prefix of any string in $\vec{y}$. Hence we can replace $\varphi_{L}\left(y_{i} \sqcap y_{j}, x\right)$ by

$$
\bigvee_{a} \bigwedge_{k} \neg \varphi_{a \Sigma^{*}}\left(y_{i} \sqcap y_{j}, y_{k}\right)
$$

where the conjunction is over all $k$ for which $y_{k}$ is an immediate successor of $y_{i} \sqcap y_{j}$ in the tree-order and the disjunction is over all symbols $a$ for which $L \cap a \Sigma^{*} \neq \emptyset$.

For the remaining two cases, we need the fact that star-free languages are closed under concatenation. Hence, for star-free languages $L^{\prime}$ and $L^{\prime \prime}$ there exists a star-free language $L$ such that the following is true: for any two strings $s_{0} \prec s_{1}$, it is the case that there is a string $s$ with $s_{0} \prec s \prec s_{1}, s-s_{0} \in L^{\prime}$ and $s_{1}-s \in L^{\prime \prime}$ iff $s_{1}-s_{0} \in L$.

The proof is straightforward from the fact that star-free languages are precisely those first-order definable in string models [54].

Next, we consider the case when $\gamma$ implies that $x$ is in the prefix closure of $\vec{y}$, but not a node of Tree $(\vec{y})$. That is, we have two nodes $s_{0}=y_{i} \sqcap y_{j}, s_{1}=y_{k} \sqcap y_{l}$ of Tree $(\vec{y})$ such that there are no other nodes of Tree $(\vec{y})$ between 
them, and $s_{0} \prec x \prec s_{1}$. Notice that any $\epsilon, \sqcap$-term $t$ in $x, \vec{y}$ that involves $x$ can be rewritten as an equivalent term $\tau$ in variables $\vec{y}$ or by $x$. Thus, there are at most two formulae of the form $\varphi_{L_{j}}$ where terms mention $x$ : these are $\varphi_{L^{\prime}}\left(s_{0}, x\right)$ and $\varphi_{L^{\prime \prime}}\left(x, s_{1}\right)$ for some star-free $L^{\prime}, L^{\prime \prime}$. Hence, $\beta(\vec{y})$ is equivalent to

$$
\gamma^{\prime}(\vec{y}) \wedge \bigwedge_{m} \varphi_{L_{m}}\left(\tau_{m}(\vec{y}), \tau_{m}^{\prime}(\vec{y})\right) \wedge \exists x\left(\left(s_{0} \prec x \prec s_{1}\right) \wedge \varphi_{L^{\prime}}\left(s_{0}, x\right) \wedge \varphi_{L^{\prime \prime}}\left(x, s_{0}\right)\right)
$$

where the big conjunction is over formulae $\varphi_{L_{j}}$ and terms do not mention $x$. By the claim, there is a star-free language $L$ such that $\exists x\left(\left(s_{0} \prec x \prec s_{1}\right) \wedge \varphi_{L^{\prime}}\left(s_{0}, x\right) \wedge \varphi_{L^{\prime \prime}}\left(x, s_{0}\right)\right)$ is equivalent to $s_{1}-s_{0} \in L$, that is, $\varphi_{L}\left(y_{i} \sqcap y_{j}, y_{k} \sqcap y_{l}\right)$, which shows that $\beta(\vec{y})$ can be put in the required form.

The last case is when $\gamma$ specifies that $x$ is not in the prefix closure of $\vec{y}$, and the meet of $x$ and Tree $(\vec{y})$ is a string $s$ between two nodes of Tree $(\vec{y})$. That is, for two consecutive nodes $s_{0}=y_{i} \sqcap y_{j}, s_{1}=y_{k} \sqcap y_{l}$ of Tree $(\vec{y})$ we have $s_{0} \prec x \sqcap s_{1} \prec s_{1}$. In particular, $x \sqcap s_{1}=x \sqcap y_{k}=x \sqcap y_{l}$. We thus have formulae $\varphi_{L_{1}}\left(s_{0}, x \sqcap y_{k}\right), \varphi_{L_{2}}\left(x \sqcap y_{k}, y_{l} \sqcap y_{k}\right)$ and $\varphi_{L^{\prime}}\left(x \sqcap y_{k}, x\right)$ as conjuncts of $\beta$, for some star-free languages $L_{1}, L_{2}, L^{\prime}$. We may assume that other subformulae of the form $\varphi_{L}$ do not mention $x$. Let $\chi(\vec{y})$ be the conjunction of all those other subformulae. Then $\beta(\vec{y})$ is equivalent to

$$
\bigvee_{a \in \Sigma} \exists z \gamma^{\prime}(\vec{y}) \wedge\left(s_{0} \prec z \prec s_{1}\right) \wedge \chi(\vec{y}) \wedge \varphi_{L_{1}}\left(s_{0}, z\right) \wedge \varphi_{L_{2} \cap\left(a \Sigma^{*}\right)}\left(z, s_{1}\right) \wedge \exists x\left(z \prec x \wedge \varphi_{L^{\prime}-a \Sigma^{*}}(z, x)\right)
$$

( $z$ plays the role of $x \sqcap s_{1}$, and the disjunction ensures that the first letters of $s_{1}-z$ and $x-z$ are different). Let $\Sigma^{\prime}=\left\{a \in \Sigma \mid L^{\prime}-a \Sigma^{*} \neq \emptyset\right\}$. Then we obtain that $\beta(\vec{y})$ is equivalent to

$$
\bigvee_{a \in \Sigma^{\prime}} \gamma^{\prime}(\vec{y}) \wedge \exists z\left(s_{0} \prec z \prec s_{1}\right) \wedge \chi(\vec{y}) \wedge \varphi_{L_{1}}\left(s_{0}, z\right) \wedge \varphi_{L_{2} \cap\left(a \Sigma^{*}\right)}\left(z, s_{1}\right)
$$

from which $z$ can be eliminated just as in the previous case. This concludes the proof.

We now give an illustration of the normal form. Suppose we have a formula $\psi(x, y)=\exists z\left(z \prec x \wedge z \prec y \wedge L_{a}(z)\right)$. In other words, there is a proper prefix of $x \sqcap y$ whose last letter is $a$. Let $L$ be the language that consists of strings that have such a prefix. It is a star-free languages, since it is definable by an FO formula over string models: $\exists i \exists j(i<$ $\left.j \wedge P_{a}(i)\right)$.

To produce the normal form for $\psi$, we consider four different possibilities for $x$ and $y: x=y, x \prec y, y \prec x$, and $x \nprec y, y \nprec x, x \neq y$, and for each we state that the meet of $x$ and $y$, in the corresponding tree, belongs to $L$. That is, the formula is:

$$
\begin{array}{rlrl} 
& ((\epsilon \prec x \wedge x=y) & \wedge & \left.\varphi_{L}(\epsilon, x)\right) \\
\vee & (\epsilon \prec x \wedge x \prec y) & \left.\wedge \varphi_{L}(\epsilon, x)\right) \\
\vee & ((\epsilon \prec y \wedge y \prec x) & \left.\wedge \varphi_{L}(\epsilon, y)\right) \\
\vee & ((\epsilon \prec x \sqcap y \wedge \neg(x \prec y) \wedge \neg(y \prec x) \wedge \neg(x=y)) & \left.\wedge \varphi_{L}(\epsilon, x \sqcap y)\right) .
\end{array}
$$

\subsubsection{Quantifier Elimination}

Let $\mathbf{S}^{+}$be the expansion of $\mathbf{S}$ to the signature that contains $\epsilon, \sqcap$ and a binary predicate $P_{L}$ for each star-free language $L$. Note that $\mathbf{S}^{+}$is a definable expansion of $\mathbf{S}$, as all additional functions and predicates are definable. From the normal form we now immediately obtain:

\section{Theorem $3.5 \mathrm{~S}^{+}$admits quantifier elimination.}

Remark. As mentioned above, there is no need to nest the $\sqcap$-operator. Therefore, $\mathbf{S}^{+}$can be turned into a relational signature that admits quantifier elimination as follows. For each star-free $L$, let $P_{L}^{\prime}$ be the set of tuples $\left(s_{1}, s_{2}, s_{3}, s_{4}\right)$ of strings for which $P_{L}\left(\sqcap\left(s_{1}, s_{2}\right), \sqcap\left(s_{3}, s_{4}\right)\right)$. Note, that $\sqcap\left(s_{1}, s_{2}\right) \preceq \sqcap\left(s_{3}, s_{4}\right)$ can be expressed as $P_{\Sigma^{*}}\left(\sqcap\left(s_{1}, s_{2}\right), \sqcap\left(s_{3}, s_{4}\right)\right)$. It is straightforward to check that this signature admits quantifier elimination. In the same way, the quantifier elimination results in the remainder of the paper can be turned into quantifier-elimination results in a relational signature.

Note also that $\mathbf{S}^{+}$could be considered as an expansion of $\mathbf{S}$ with either functions $l_{a}$ or predicates $L_{a}$ in the signature. In the latter case, predicates $L_{a}$ are not needed as $L_{a}(x)$ iff $P_{\Sigma^{*} a}(\epsilon, x)$. 
Another corollary of the normal form is that in the language of $\mathbf{S}$, it suffices to use only bounded quantification. That is, we introduce bounded quantifiers of the form $\exists x \preceq y$ and $\forall x \preceq y$ (where $\exists x \preceq y \varphi$ means $\exists x x \preceq y \wedge \varphi$ ), and let $\mathrm{FO}_{b}(\mathbf{S})$ be the restriction of $\mathrm{FO}(\mathbf{S})$ to formulae $\varphi\left(y_{1}, \ldots, y_{k}\right)$ in which all quantifiers are of the form $Q x \preceq y_{i}$. From the normal form and the fact that each $\varphi_{L}$ can be defined with bounded quantifiers, we obtain:

Corollary 3.6 $\mathrm{FO}_{b}(\mathbf{S})=\mathrm{FO}(\mathbf{S})$.

Finally, we characterize $\mathbf{S}$-definable subsets of $\Sigma^{*}$ and $\left(\Sigma^{*}\right)^{k}$. Given a subset $R \subseteq\left(\Sigma^{*}\right)^{k}$ and a permutation $\pi$ on $\{1, \ldots, k\}$, by $\pi(R)$ we mean the set $\left\{\left(s_{\pi(1)}, \ldots, s_{\pi(k)}\right) \mid\left(s_{1}, \ldots, s_{k}\right) \in R\right\}$.

\section{Corollary 3.7}

a) A language $L \subseteq \Sigma^{*}$ is definable in $\mathbf{S}$ iff it is star-free.

b) The class of relations definable over $\mathrm{FO}(\mathbf{S})$ is the minimal class containing the empty set, $\{\epsilon\},\{a\}$, for $a \in \Sigma$, $\preceq, \sqcap$, and closed under Boolean operations, Cartesian product, permutation, and the operation $*$ defined by $L_{1} * L_{2}=\left\{\left(s_{1}, s_{1} \cdot s_{2}\right) \mid s_{1} \in L_{1}, s_{2} \in L_{2}\right\}$ for $L_{1}, L_{2} \subseteq \Sigma^{*}$.

Proof. a) $\mathbf{S}^{+}$formulae in one free variable are Boolean combinations of $P_{L}(\epsilon, x)$, for $L$ star-free, and thus they define only star-free languages.

b) For one direction notice that $\epsilon,\{a\}, \prec, \sqcap$ are definable in $\operatorname{FO}(\mathbf{S})$, and that $\mathrm{FO}(\mathbf{S})$ is closed under Boolean operations, permutation and Cartesian product. The closure under $*$ is an easy consequence of Lemma 3.3 as $L_{1} * L_{2}$ corresponds to $\left\{(x, y) \mid \varphi_{L_{1}}(\epsilon, x) \wedge \varphi_{L_{2}}(x, y)\right\}$. The other direction follows from the normal form.

Note that the projection operation is not needed in the closure result above.

\subsubsection{Automata}

We now give an automaton model characterizing definability in $\mathrm{FO}(\mathbf{S})$. This automaton model corresponds exactly to the counter-free variant of regular prefix automaton as defined in [4].

Let us recall the definition of regular prefix automata. Let $A$ be a finite non-deterministic automaton on strings with state set $Q$, transition relation $\delta$ and initial state $q_{0}$. We construct from $A$ an automaton $\hat{A}=\left(\Sigma, Q, q_{0}, F, \delta\right)$ accepting $n$-tuples $\vec{w}=\left(w_{1}, \cdots, w_{n}\right)$ of strings in the following way. $F$ is a subset of $Q^{n}$ which denotes the accepting states of $\hat{A}$. Let $\operatorname{prefix}(\vec{w})$ be the set of all prefixes of all $w_{i}$. A run of $\hat{A}$ over $\vec{w}$ is a mapping $h$ from $\operatorname{prefix}(\vec{w})$ to $Q$ which assigns to every node $\alpha \in \operatorname{prefix}(\vec{w})$ a state $q \in Q$ such that $h(\epsilon)=q_{0}$ and, $\beta=l_{a}(\alpha)$ implies $h(\beta) \in \delta(h(\alpha), a)$. The run is accepting if $\left(h\left(w_{1}\right), \cdots, h\left(w_{n}\right)\right) \in F$. The $n$-tuple $\vec{w}$ is accepted by $\hat{A}$ if there is an accepting run of $\hat{A}$ over $\vec{w}$. See [4] for more details.

For each finite non-deterministic automaton $A$ a corresponding automaton $\hat{A}$ is called a regular prefix automaton (RPA). The subset of $\left(\Sigma^{*}\right)^{n}, n \in \mathbb{N}$, it defines is called a regular prefix relation (RPR).

We say that $\hat{A}$ is counter-free (CF-PA) if $A$ is counter-free. The following shows that the relations definable in $\mathrm{FO}(\mathbf{S})$ are exactly those recognizable by a CF-PA.

Proposition 3.8 A relation is definable in $\mathrm{FO}(\mathbf{S})$ if and only if it is definable by a counter-free prefix automaton.

Proof. One direction follows from Corollary 3.7 as it is easy to verify that counter-free prefix automata can recognize the empty set, $\{\epsilon\},\{a\} a \in \Sigma,\{(u, v) \mid u \preceq v\},\{(u, v, w) \mid u \sqcap v=w\}$, and are closed under Boolean operations, Cartesian product, permutation, and $*$.

For the opposite direction let $\hat{A}$ be a CF-PA accepting the relation $R$ of arity $n$. We show that $R$ can be defined by an $\mathrm{FO}(\mathbf{S})$ formula $\varphi$. Let $Q$ be the set of states of $A$. If $q_{1}, q_{2}$ are two states in $Q$, let $L\left(q_{1}, q_{2}\right)$ be the set of strings $w$ such that $A$ can get from state $q_{1}$ to state $q_{2}$ by reading $w$. Because $A$ is counter-free $L\left(q_{1}, q_{2}\right)$ is a star-free language.

The formula $\varphi$ is a disjunction over formulae $\gamma(\vec{x}) \wedge \psi_{\gamma}(\vec{x})$, where $\gamma$ cycles through all complete tree-order descriptions. Each formula $\psi_{\gamma}(\vec{x})$ is a disjunction over all possible assignments of states to the (at most $2 n$ ) strings of $\operatorname{Tree}(\vec{x})$. For each such assignment it checks that the vector of states at $\vec{x}$ is accepting and that the states are consistent, i.e., that, for each pair $(y, z)$ of successive elements of Tree $(\vec{x})$, the path from $y$ to $z$ fulfills $P_{L}\left(q_{1}, q_{2}\right)$ where $q_{1}$ and $q_{2}$ are the states at $y$ and $z$ in the assignment under consideration, respectively. 


\subsubsection{VC-dimension and Isolation}

We defined the notions of isolation and VC dimension in Section 2; these notions are very important for the database part of the paper, as they provide strong bounds on the expressiveness of various relational calculi. The notion of finite VC-dimension, coming originally from statistics and machine learning [5], is of independent interest, as it states that families definable over some structures on strings could be learned effectively.

We have seen that $\mathbf{S}_{\text {len }}$ has infinite VC-dimension. It turns out that all other structures we consider here, have finite VC-dimension. To prove this, we have to introduce some new machinery, which is presented next. After that, we show that $\mathbf{S}$ has finite $\mathrm{VC}$-dimension.

Lemma 3.9 Let $M$ be a model with the isolation property. Then its definable families have finite VC-dimension.

Proof. We give two proofs of this result, one is complexity-theoretic and one is model-theoretic. We start with the complexity-theoretic proof. Assume that $M$ does not have finite VC dimension. By [51] it has the independence property, and by [63], there is a single formula $\varphi(\vec{x}, \vec{y})$ (in fact, $\varphi(\vec{x}, y)$ ) that has the independence property: that is, for every $n$, there is a set $F_{n} \subseteq M$ of size $n$ such that for every $X \subseteq F_{n}$, there is $\vec{x}_{X}$ such that for any $y_{0} \in F_{n}$, $\varphi\left(\vec{x}_{X}, y_{0}\right)$ iff $y_{0} \in X$.

Next consider an expansion of $M$ with one unary predicate $U$, and one binary predicate $E$. Let $\Phi$ be

$$
\begin{aligned}
& \forall v, w(E(v, w)\rightarrow(U(v) \wedge U(w))) \\
& \wedge \quad \neg \exists \vec{s}_{1}, \vec{s}_{2}\left(\begin{array}{rl} 
& \forall v U(v) \leftrightarrow\left(\varphi\left(\vec{s}_{1}, v\right) \vee \varphi\left(\vec{s}_{2}, v\right)\right) \\
\wedge \quad \forall v, w\left(U(v) \wedge U(w) \wedge \varphi\left(\vec{s}_{1}, v\right) \wedge \varphi\left(\vec{s}_{2}, w\right)\right) \rightarrow \neg E(v, w)
\end{array}\right) .
\end{aligned}
$$

The first conjunct says that $E$ is a graph whose nodes are in the set $U$. The second says that, assuming $U \subseteq F_{n}$, there cannot be two subsets of $U$ such that there are no $E$-edges between them. Thus, if $U$ is a finite subset of $F_{n}, \Phi$ says that $E$ is connected.

The isolation property $[8,32]$ implies that $\Phi$ can be expressed by a sentence $\Psi$ of the form $Q z_{1} \in U \ldots Q z_{l} \in$ $U \alpha(\vec{z})$ over all finite $U$, where $\alpha$ is a Boolean combination of $E, U$-atomic formulae, and formulae $\gamma(\vec{z})$ in the language of $M$.

Next, for each $n$, fix a 1-to-1 mapping $\pi:\{1, \ldots, n\} \rightarrow F_{n}$ and for each $\gamma$ appearing in $\Psi$, define $P_{\gamma}^{n}(\vec{z})$ on $\{1, \ldots, n\}$ to contain all the tuples $\vec{n}$ such that $\gamma(\pi(\vec{n}))$ is true. Let then $\Psi_{n}$ be the sentence in the language of $E$ and all $P_{\gamma}^{n}$ of the form $Q z_{1} \ldots Q z_{l} \alpha^{\prime}$ where $\alpha^{\prime}$ is obtained from $\alpha$ by replacing each $U(\cdot)$ by true, and each $\gamma(\vec{z})$ by $P_{\gamma}^{n}(\vec{z})$. It then follows that for a graph $E$ on $\{1, \ldots, n\}, E \models \Psi_{n}$ iff $E$ is connected. However, this implies that connectivity is in non-uniform $\mathrm{AC}^{0}$, which is false [26]. This concludes the proof.

Second proof. We now give another, model-theoretic proof. For a formula $\varphi(\vec{x}, \vec{y})$ and set $A \subseteq M$, a $\varphi$-type over $A$ is a maximal consistent (w.r.t. $\operatorname{Th}(M)$ ) set of formulae of the form $\varphi(\vec{x}, \vec{a})$ with $\vec{a}$ a tuple over $A$. For $\vec{c}$ in $M$ and $A$ as above, we can then talk about the $\varphi$-type of $\vec{c}$ over $A$, denoted $t p_{\varphi}(\vec{c} / A)$.

Let $\varphi(\vec{x}, \vec{y})$ be a formula over $M$. We next show that there are integers $n$ and $K$ such that for any finite set $A$, there are at most $K|A|^{n} \varphi$-types over $A$.

To prove this we first claim that for each $\varphi$ there is a formula $\gamma_{\varphi}(\vec{x}, \vec{z})$ and an integer $n$ such that for every finite set $A$, and any vector $\vec{s}$, there is an $n$-element subset $X$ of $A$ such that $t p_{\varphi}(\vec{s} / A)$ is isolated by $t p_{\gamma_{\varphi}}(\vec{s} / X)$.

Indeed, assume that for some $\varphi$ there was no such $n$ and $\gamma$. Then for each $\gamma$ and each $n$ there exists a finite set $A_{\gamma}^{n}$ and a vector $\vec{s}_{\gamma}^{n}$ such that for any finite subset $X$ of $A_{\gamma}^{n}$ of size $<n, t p_{\varphi}\left(\vec{s}_{\gamma}^{n} / A_{\gamma}^{n}\right)$ is not isolated by $t p_{\gamma}\left(\vec{s}_{\gamma}^{n} / X\right)$. Then, by compactness, we get a pseudo-finite set $W_{\gamma}$ (the ultraproduct of the $\left(A_{\gamma}^{n}\right)_{n \in \mathbb{N}}$ ) and a vector $\vec{s}_{\gamma}$ (the ultraproduct of the $\left.\left(\vec{s}_{\gamma}^{n}\right)_{n \in \mathbb{N}}\right)$ in a model of $\operatorname{Th}(M)$ such that for any finite set $X$ of $W_{\gamma}, t p_{\varphi}\left(\vec{s}_{\gamma} / W_{\gamma}\right)$ is not isolated by $t p\left(\vec{s}_{\gamma} / X\right)$. Then, by compactness again, we get another model of $\operatorname{Th}(M)$ with a pseudo-finite set $W$ and $\vec{s}$, such that for any countable subset $X$ of $W, t p(\vec{s} / W)$ is not isolated by $\operatorname{tp}(\vec{s} / X)$, which contradicts isolation.

Now let $K$ be $2^{n^{|\vec{z}|}}$. It is easy to see that $n$ and $K$ work. There are at most $|A|^{n}$ subsets $X$ from $A$ of size $n$. For each fixed set $X$ of size $n$, there are at most $n^{|\vec{z}|}$ formulae of the form $\gamma(\vec{x}, \vec{e})$ with $\vec{e} \in X$, and hence there are at most $K \gamma$-types over $X$. Since the $\varphi$-type of a vector $\vec{c}$ from $M$ is determined by the choice of the set $X$ whose $\gamma$-type isolates it and the $\gamma$-type of $\vec{c}$ over $X$, it follows that there are at most $K|A|^{n}$ types. 
Now let $\mathcal{C}$ be the family definable by $\varphi(\vec{x}, \vec{y})$. If a finite set $A$ is shattered by members of $\mathcal{C}$, then the number of $\varphi$-types over $A$ is $2^{|A|}$. Hence, arbitrarily large finite sets cannot be shattered by $\mathcal{C}$.

Next, we show the following.

Proposition 3.10 $\mathrm{Th}(\mathbf{S})$ has the strong isolation property.

Proof. Let $M$ be a model of $\operatorname{Th}(\mathbf{S}), W$ be a pseudo-finite set of elements of $M$, and $a \in M$. We exhibit a finite subset $W_{0}$ of $W$ such that $t_{M}\left(a / W_{0}\right)$ isolates $t p_{M}(a / W)$.

Note that for each finite set $X$, the elements $\operatorname{Meet}(a, X)$, Meet $-(a, X)$ and Meet $_{+}(a, X)$ can be described by means of formulae of $\mathrm{FO}(\mathbf{S})$ : $\operatorname{Meet}(a, X)$ is the largest prefix of $a$ which is in the prefix closure of $X$, and Meet $-(a, X)$, Meet $+(a, X)$ are the nodes of Tree $(X)$ (meets of two elements of $X$ ) which are closest to $\operatorname{Meet}(a, X)$. Hence, such elements exist for $W$, since $W$ is pseudo-finite. Let $w_{1}, w_{2}, w_{3}, w_{4} \in W$ be such that $w_{1} \sqcap w_{2}=$ Meet $_{-}(a, W)$ and $w_{3} \sqcap w_{4}=\operatorname{Meet}_{+}(a, W)$. Take $W_{0}=\left\{w_{1}, w_{2}, w_{3}, w_{4}\right\}$.

We know that any formulae of $\mathrm{FO}(\mathbf{S})$ can be put in the normal form described in Proposition 3.4. Thus a type of $a$ over $W$ is entirely defined by the tree structure of $a \cup W$ and the paths between definable nodes of that tree. If we fix $W$, we conclude that the paths between $\operatorname{Meet}(a, W)$, Meet_ $(a, W)$, $\operatorname{Meet}_{+}(a, W)$ and $a$ completely define $t p_{M}(a / W)$. Because $t p_{M}\left(a / W_{0}\right)$ already describes all the paths between $\operatorname{Meet}(a, W), \operatorname{Meet}_{-}(a, W), \operatorname{Meet}_{+}(a, W)$ and $a$, the result follows.

Combining Proposition 3.10 and Lemma 3.9, we conclude that the model $\mathbf{S}$, unlike $\mathbf{S}_{\text {len }}$, has learnable definable families.

Corollary 3.11 Every definable family in $\mathbf{S}$ has finite VC-dimension.

\subsection{A star-free algebra based on $\mathrm{S}_{\text {left }}$}

We now study an example of a star-free algebra, in which the $n$-ary relations in the algebra are more complex than those definable over $\mathbf{S}$. Recall that $\mathbf{S}_{\text {left }}=\left\langle\Sigma^{*}, \preceq,\left(l_{a}\right)_{a \in \Sigma},\left(f_{a}\right)_{a \in \Sigma}\right\rangle$; that is, in this structure one can add characters on the right as well as on the left.

Without the prefix relation, this structure was studied in $[16,60]$, as a model of queues. A quantifier-elimination result was proved in [60], by extending quantifier-elimination for term algebras (in fact [60] showed that term algebras with queues admit QE). However, as in the case of $\mathbf{S}$, which differs from strings as terms algebras in that it has the prefix relation, the prefix relation complicates things considerably.

We start with the easy observation that $\mathrm{FO}\left(\mathbf{S}_{\text {left }}\right)$ expresses more relations that $\mathrm{FO}(\mathbf{S})$. Indeed, the graph of $f_{a}$, $F_{a}=\left\{(x, a \cdot x) \mid x \in \Sigma^{*}\right\}$ is not expressible in $\mathrm{FO}(\mathbf{S})$, which can be shown by a simple game argument. More precisely, given a number $k$ of rounds, let $n=2^{k}+1$ and consider the game on the tuples $\left(0^{n}, 10^{n}\right)$ and $\left(0^{n+1}, 10^{n}\right)$. By Corollary 3.6 it is sufficient to play on the prefixes of the participating strings. The duplicator has a trivial winning strategy on the strings $10^{n}$ and a well-known winning strategy on $0^{n}$ versus $0^{n+1}$.

\subsubsection{Quantifier Elimination}

Let $\mathbf{S}_{\text {left }}^{+}$be the extension of $\mathbf{S}_{\text {left }}$ with the same (definable) functions and predicates we added to $\mathbf{S}^{+}$(that is, a constant $\epsilon$ for the empty string, the binary function $\sqcap$ for the longest common prefix, the predicate $P_{L}(x, y)$ for each star-free language $L$ ), and the unary function $x \mapsto x-a$, for each $a \in \Sigma$ (which is also definable).

Theorem 3.12 $\mathrm{S}_{\text {left }}^{+}$admits quantifier elimination.

In the rest of the section, we prove Theorem 3.12. Let $\Omega_{\mathbf{S}^{+}}$and $\Omega_{\mathbf{S}_{\text {left }}^{+}}$be the first-order signature of $\mathbf{S}^{+}$and $\mathbf{S}_{\text {left }}^{+}$, respectively. Let $M$ be an $\omega$-saturated model over $\Omega_{\mathbf{S}_{\text {left }}^{+}}$elementary equivalent to $\mathbf{S}_{\text {left }}^{+}$. It suffices to prove quantifier elimination in $M$. Note that $M$ can have both finite and infinite strings.

We next need the following standard result: 
Claim 1 If there exists a formula which does not admit quantifier elimination in $M$, then there exist two tuples of elements in $M$ which have the same atomic type but not the same type.

Proof of Claim 1. Let $\varphi(\vec{x}) \in \mathrm{FO}\left(\mathbf{S}_{\text {left }}^{+}\right)$, and let $Q$ enumerate all quantifier free formulae over $\Omega_{\mathbf{S}_{\text {left }}^{+}}$realizable in $M$. Let $\Gamma_{\varphi}\left(\vec{x}_{1}, \vec{x}_{2}\right)$ be the type asserting $\bigwedge_{\psi \in Q}\left(\psi\left(\vec{x}_{1}\right) \leftrightarrow \psi\left(\vec{x}_{2}\right)\right) \wedge \neg\left(\varphi\left(\vec{x}_{1}\right) \leftrightarrow \varphi\left(\vec{x}_{2}\right)\right)$.

We show that if $\varphi$ is not equivalent to a quantifier-free formula then $\Gamma_{\varphi}$ is satisfied in $M$. Towards a contradiction assume $\Gamma_{\varphi}$ is not satisfied in $M$. Since $M$ is $\omega$-saturated, by compactness it follows that there is a finite set $J \subseteq Q$ such that

$$
\forall \vec{x}_{1} \forall \vec{x}_{2}\left[\left(\bigwedge_{i \in J} \psi_{i}\left(\vec{x}_{1}\right) \leftrightarrow \psi_{i}\left(\vec{x}_{2}\right)\right) \rightarrow\left(\varphi\left(\vec{x}_{1}\right) \leftrightarrow \varphi\left(\vec{x}_{2}\right)\right)\right]
$$

holds in $M$. For $K \subseteq J$ let $\chi_{K}$ be $\bigwedge_{i \in K} \psi_{i} \wedge \bigwedge_{i \in J-K} \neg \psi_{i}$.

Let $G$ be $\left\{I \subseteq J \mid M \models \forall \vec{x} \chi_{I}(\vec{x}) \rightarrow \varphi(\vec{x})\right\}$ and $\rho=\bigvee_{I \in G} \chi_{I}$. To get a contradiction we show that $\rho$ is equivalent to $\varphi$ in $M$. Let $\vec{c}$ be a tuple of $M$ with $M \models \varphi(\vec{c})$. Let $L=\left\{i \in J \mid M \models \psi_{i}(\vec{c})\right\}$. If a tuple $\vec{d}$ from $M$ satisfies $\chi_{L}$ then for each $i \in J, M \models \psi_{i}(\vec{c}) \leftrightarrow \psi_{i}(\vec{d})$. By the choice of $J$ we can conclude that $M \models \varphi(\vec{c}) \leftrightarrow \varphi(\vec{d})$, hence $M \models \varphi(\vec{d})$. Therefore $L \in G$ and $M \models \rho(\vec{c})$. On the other hand, by the definition of $G$ and $\rho$ it follows immediately that $M \models \rho(\vec{c})$ implies $M \models \varphi(\vec{c})$. Hence, $\varphi$ and $\rho$ are equivalent in $M$, the desired contradiction. The claim is proved.

Thus, to prove QE, we must show that every two tuples of elements of $M$ that have the same atomic type, have the same type.

Define a nice term of $\Omega_{\mathbf{S}_{\text {left }}^{+}}$as a term of the form $t(x)=x-a+b$ (meaning $\left.(x-a)+b\right)$, where $a$ and $b$ are finite strings.

We define two relations $\equiv$ and $\equiv_{1}$ on tuples (of the same length) of strings as follows.

- $\vec{c} \equiv \vec{d}$ for $n$-tuples $\vec{c}$ and $\vec{d}$ iff for all sequences $i_{1}, \ldots, i_{k}$ from $\{1, \ldots, n\}$ and all sequences $t_{1}, \ldots, t_{k}$ of nice terms:

$$
\operatorname{atp}_{\mathbf{S}^{+}}\left(t_{1}\left(c_{i_{1}}\right), \ldots, t_{k}\left(c_{i_{k}}\right)\right)=a t p_{\mathbf{S}^{+}}\left(t_{1}\left(d_{i_{1}}\right), \ldots, t_{k}\left(d_{i_{k}}\right)\right) .
$$

- $\left(c^{\prime}, \vec{c}\right) \equiv_{1}\left(d^{\prime}, \vec{d}\right)$ for $n$-tuples $\vec{c}, \vec{d}$ and strings $c^{\prime}, d^{\prime}$ iff for all sequences $i_{1}, \ldots, i_{k}$ from $\{1, \ldots, n\}$ and all sequences $t_{1}, \ldots, t_{k}$ of nice terms:

$$
\operatorname{atp}_{\mathbf{S}^{+}}\left(c^{\prime}, t_{1}\left(c_{i_{1}}\right), \ldots, t_{k}\left(c_{i_{k}}\right)\right)=a t p_{\mathbf{S}^{+}}\left(d^{\prime}, t_{1}\left(d_{i_{1}}\right), \ldots, t_{k}\left(d_{i_{k}}\right)\right) .
$$

Of course, $\left(c^{\prime}, \vec{c}\right) \equiv\left(d^{\prime}, \vec{d}\right)$ implies $\left(c^{\prime}, \vec{c}\right) \equiv_{1}\left(d^{\prime}, \vec{d}\right)$, as the identity is a nice term. We will show that these two relations coincide.

We will show in Lemma 3.14 a stronger result than what is needed by Claim 1 in order to prove Theorem 3.12. Indeed we will show that $\equiv$ has the back-and-forth property. In order to simplify the strategy for the $\equiv$ game we first show in Lemma 3.13 that it is enough to have a strategy for the $\equiv_{1}$ game. Lemma 3.13 is proved by rewriting rules on the atomic formulas that get rid of nice terms containing $c^{\prime}$.

Lemma 3.13 If $\left(c^{\prime}, \vec{c}\right) \equiv_{1}\left(d^{\prime}, \vec{d}\right)$, then also $\left(c^{\prime}, \vec{c}\right) \equiv\left(d^{\prime}, \vec{d}\right)$.

Once the equivalence of $\equiv_{1}$ and $\equiv$ is established, we will show that they have the back-and-forth property, from which quantifier-elimination will follow.

Proof of Lemma 3.13. We start with a few observations. It is easy to see that for every atomic formula of $\mathrm{FO}\left(\mathbf{S}_{\text {left }}^{+}\right)$, there is an equivalent $\mathrm{FO}\left(\mathbf{S}_{\text {left }}^{+}\right)$formula in which every term is a meet of two nice terms (addition and subtraction of $t_{1} \sqcap t_{2}$ can be pushed back into $t_{1}$ and $t_{2}$, while multiple meets can be eliminated by adding disjunctions of treeordering formulae considering all possible cases). Notice also that atomic formulae of the form $t \preceq t^{\prime}$ where $t$ and $t^{\prime}$ are terms are equivalent to $P_{\Sigma^{*}}\left(t, t^{\prime}\right)$, and $t \prec t^{\prime}$ is equivalent to $P_{\Sigma^{+}}\left(t, t^{\prime}\right)$. Thus, we can assume that no symbols $\preceq$ and $\prec$ occur.

We call a nice term $t(x)=x-a+b$ empty if $a=b=\epsilon$.

The proof of Lemma 3.13 is done by rewriting atomic formulas in order to get rid of nice terms from one of the variables. We will proceed by a case analysis based on the rewriting rules presented in the next 4 claims. 
The first claim shows how to replace a single nice terms from a distinguished variable $s^{\prime}$. The proof is straightforward.

Claim 2 1. Let $s, s^{\prime}$ be in $M$ and let $a, b$ be finite strings and let $L$ be star-free. Then $P_{L}\left(s, s^{\prime}-a+b\right)$ is true in $M$ iff one of the following conditions holds.

- $s \preceq b$, and $s^{\prime}-a+(b-s) \in L$

- $a \preceq s^{\prime}, b \preceq s$ and $P_{L}\left(s-b+a, s^{\prime}\right)$.

Notice that in the first case above $s$ is finite, and thus the condition over $s^{\prime}$ is expressible in $\left.\mathrm{FO}(\mathrm{S})\right)$.

2. Let $s, s^{\prime}$ be in $M$ and let $a, b$ be finite strings and let $L$ be star-free. Then $P_{L}\left(s-a+b, s^{\prime}\right)$ is true in $M$ iff one of the following conditions holds.

- $a \npreceq s, b \preceq s^{\prime}$ and $s^{\prime}-b \in L$;

- $a \preceq s$ and $P_{L}\left(s, s^{\prime}-b+a\right)$.

The next claim shows how to get rid of terms of the form $t(s) \sqcap s$ from distinguished variable $s$.

Claim 3 Let $s$ be an element of $M$, t a nice term over $\Omega_{\mathbf{S}_{\text {left }}^{+}}$. Let $s^{\prime}=t(s) \sqcap s$. There is a quantifier-free $\mathrm{FO}\left(\mathbf{S}^{+}\right)$ formula $\varphi_{s, t}(x, y)$ such that $\varphi_{s, t}\left(s, s^{\prime}\right)$ and $\forall x \varphi_{s, t}(x, y) \rightarrow y=x \sqcap t(x)$ hold in $M$.

Proof of Claim 3. Let $a, b$ finite strings such that $t(x)=x-a+b$. If $s^{\prime}=s \sqcap(s-a+b)$ is finite, then $\varphi_{s, t}(x, y)$ is $s^{\prime}=(x \sqcap(x-a+b)) \wedge y=s^{\prime}$. Here, $s^{\prime}=x \sqcap(x-a+b)$ can be expressed in $\mathrm{FO}(\mathbf{S})$ by $\left(s^{\prime} \preceq x\right) \wedge\left(s^{\prime}-b+a \preceq\right.$ $x) \wedge \bigwedge_{\alpha \in \Sigma} \neg\left(s^{\prime} \cdot \alpha \preceq x \wedge s^{\prime} \cdot \alpha-b+a \preceq x\right)$. If $s^{\prime}$ is infinite, then let $n=|a|$ and $m=|b|$. We have $b \preceq s, a \preceq s$, and $s[n+i]=s[m+i]$ for $i \in \mathbb{N}$. For given $n, m$ it is possible to define an $\mathrm{FO}(\mathbf{S})$ formula $\psi(x, y)$ which is true if and only if $y$ is maximal such that $y \preceq x,|y|>m$, and $x(n+i)=x(m+i)$, where $i=|y|-m$. Then we let $\varphi_{s, t}(x, y)$ be $a \preceq x \wedge b \preceq x \wedge \psi(x, y)$. It is easy to verify that $\varphi_{s, t}\left(s, s^{\prime}\right)$ holds and that $\varphi_{s, t}(x, y)$ implies $y=x \sqcap t(x)$. Finally, by quantifier-elimination in $\mathrm{FO}\left(\mathbf{S}^{+}\right) \varphi_{s, t}$ can be made quantifier-free.

The following is the analog of the preceding claim for terms of the form $t\left(s \sqcap s^{\prime}\right)$.

Claim 4 Let $t, t^{\prime}$ be nice terms and L star-free. Assume that there are strings $s, s^{\prime}, s^{\prime \prime}$ such that $P_{L}\left(t\left(s \sqcap s^{\prime}\right), t^{\prime}\left(s \sqcap s^{\prime \prime}\right)\right)$ holds. Then there is an $\mathrm{FO}(\mathbf{S})$ formula $\rho(x, y, z)$ such that $\rho\left(s, s^{\prime}, s^{\prime \prime}\right)$ holds and such that, for all $r, r^{\prime}, r^{\prime \prime}$ in $M$, $\rho\left(r, r^{\prime}, r^{\prime \prime}\right)$ implies $P_{L}\left(t\left(r \sqcap r^{\prime}\right), t^{\prime}\left(r \sqcap r^{\prime \prime}\right)\right)$.

Proof of Claim 4. Let $t(x)=x-a+b$ and $t^{\prime}(x)=x-a^{\prime}+b^{\prime}$. First of all, if $P_{L}\left(t\left(s \sqcap s^{\prime}\right), t^{\prime}\left(s \sqcap s^{\prime \prime}\right)\right)$ holds then from Claim 2 we have either:

1. $t\left(s \sqcap s^{\prime}\right) \preceq b^{\prime}$ and $\left(s \sqcap s^{\prime \prime}\right)-a^{\prime}+\left(b^{\prime}-t\left(s \sqcap s^{\prime}\right)\right) \in L$, or

2. $a^{\prime} \preceq s \sqcap s^{\prime \prime}, b^{\prime} \preceq t\left(s \sqcap s^{\prime}\right)$, and $P_{L}\left(t\left(s \sqcap s^{\prime}\right)-b^{\prime}+a^{\prime}, s \sqcap s^{\prime \prime}\right)$.

Consider the first case. Notice that it implies that $t\left(s \sqcap s^{\prime}\right)$ is a finite string. Hence, the second condition says that $s \sqcap s^{\prime \prime} \in L^{\prime}$, for the star-free set $L^{\prime}$ of strings $z$ with $z-a^{\prime}+\left(b^{\prime}-t\left(s \sqcap s^{\prime}\right)\right) \in L$. The first condition holds iff (a) $a$ is not a prefix of $s \sqcap s^{\prime}$ and $b \preceq b^{\prime}$ or (b) $s \sqcap s^{\prime}$ is finite in which case $t\left(s \sqcap s^{\prime}\right) \preceq b^{\prime}$ can be easily expressed in $\mathrm{FO}(\mathbf{S})$.

Consider now the second case. The conditions $a^{\prime} \preceq s \sqcap s^{\prime \prime}$ and $b^{\prime} \preceq t\left(s \sqcap s^{\prime}\right)$ can be easily expressed in $\mathrm{FO}(\mathbf{S})$. It remains to express $P_{L}\left(t\left(s \sqcap s^{\prime}\right)-b^{\prime}+a^{\prime}, s \sqcap s^{\prime \prime}\right)$. As before, we can assume that the first term is nice, i.e., we only have to show how $P_{L}\left(t\left(s \sqcap s^{\prime}\right), s \sqcap s^{\prime \prime}\right)$, where $t(x)=x-a+b$, can be expressed.

We distinguish two subcases.

If $t\left(s \sqcap s^{\prime}\right)$ is finite then the corresponding $\mathrm{FO}(\mathbf{S})$ formula is obtained similarly to the previous case.

Assume now $t\left(s \sqcap s^{\prime}\right)$ infinite. In this case, as $s \sqcap s^{\prime}$ is a prefix of $s$ (and therefore $s \sqcap s^{\prime} \preceq s \sqcap s^{\prime \prime}$ or $s \sqcap s^{\prime \prime} \preceq s \sqcap s^{\prime}$ holds), it is sufficient to express that the suffix of $s \sqcap s^{\prime \prime}$ relative to its prefix of length $\left|s \sqcap s^{\prime}\right|-|b|+|a|$ is in $L$. This can clearly be expressed in $\mathrm{FO}(\mathbf{S})$.

Let $\varphi(x, y)$ be an $\mathrm{FO}(\mathbf{S})$ formula. If in $M$, there is at most one $s^{\prime}$ for each $s$ such that $\varphi\left(s, s^{\prime}\right)$ holds, then we call $\varphi$ functional, as $\varphi$ defines a partial function $f_{\varphi}$ on $\mathrm{M}$ by $f_{\varphi}(s)=s^{\prime}$ if $\varphi\left(s, s^{\prime}\right)$ holds. Note that $\varphi_{s, t}$ of Claim 3 is 
functional. We call a term of the form $f_{\varphi}(x)$ where $\varphi$ is functional a $\Omega_{\mathbf{S}}$-function term, if for each $s$ in $M, f_{\varphi}(s) \preceq s$. Let $\Omega_{\mathbf{S}_{\text {left }}^{++}}$be the signature obtained from $\Omega_{\mathbf{S}_{\text {left }}^{+}}$by adding all $\Omega_{\mathrm{S}}$-function terms.

The next claim shows that in attempting to eliminate terms with "-" from distinguished variable $y$, it suffices to deal with terms of a particularly simple form.

Claim 5 Let $s$ be an element of $M$. For every atomic $\mathrm{FO}\left(\mathbf{S}_{\text {left }}^{+}\right)$formula $\varphi(y, \vec{x})$ there is a quantifier-free $\mathrm{FO}\left(\mathbf{S}_{\text {left }}^{++}\right)$ formula $\varphi^{\prime}(y, \vec{x})$, such that for all $\vec{r}$ from $M, \varphi(s, \vec{r})$ holds if and only if $\varphi^{\prime}(s, \vec{r})$ holds. We can also ensure that $y$ appears in $\varphi^{\prime}$ only in terms of the form $t\left(y \sqcap t^{\prime}\left(x_{i}\right)\right)$, where $t$ and $t^{\prime}$ are nice terms, and in $\Omega_{\mathbf{S}}$-function terms $t\left(f_{\varphi}(y)\right)$. Furthermore, we can arrange that $\Omega_{\mathbf{S}}$-function terms in y are the only $\Omega_{\mathbf{S}}$-function terms in $\varphi^{\prime}$.

Proof of Claim 5. As mentioned before, we can assume w.l.o.g. that $\varphi$ only contains terms of the form $t_{1}\left(v_{1}\right) \sqcap t_{2}\left(v_{2}\right)$, where $t_{1}, t_{2}$ are nice and $v_{1}, v_{2}$ are from $y, \vec{x}$. We first show, that every atomic formula $\psi\left(t(y) \sqcap t^{\prime}\left(x_{i}\right), t^{\prime \prime}(y, \vec{x})\right)$ can be replaced by an equivalent formula

$$
\psi^{\prime}(y, \vec{x})=\bigvee_{j} \psi_{j}\left(y, x_{i}\right) \wedge \psi\left(t_{j}\left(y \sqcap t_{j}^{\prime}\left(x_{i}\right)\right), t^{\prime \prime}(y, \vec{x})\right),
$$

where the $\psi_{j}$ are quantifier-free $\mathrm{FO}(\mathbf{S})$ formulae and the $t_{j}, t_{j}^{\prime}$ are nice terms.

Let $t(y)$ be $y-a+b$ and $t^{\prime}\left(x_{i}\right)$ be $x_{i}-a^{\prime}+b^{\prime}$. To prove the above statement we consider three cases.

Case $1 b \preceq b^{\prime}$. Then $y-a+b \sqcap x_{i}-a^{\prime}+b^{\prime}$ is $b$ if $a \npreceq y$ and $\left(y \sqcap\left(x_{i}-a^{\prime}+\left(b^{\prime}-b\right)+a\right)\right)-a+b$, otherwise.

Case $2 b^{\prime} \prec b$. Then $y-a+b \sqcap x_{i}-a^{\prime}+b^{\prime}$ is $\left.\left(y-a+\left(b-b^{\prime}\right)\right) \sqcap x_{i}-a^{\prime}\right)+b^{\prime}$. There are two subcases. Either $\left(b-b^{\prime}\right) \preceq\left(x_{i}-a^{\prime}\right)$ and then $y-a+b \sqcap x_{i}-a^{\prime}+b^{\prime}$ is $\left((y-a) \sqcap\left(x_{i}-a^{\prime}-\left(b-b^{\prime}\right)\right)\right)+b$ and we proceed as in case 1. Otherwise $b \npreceq x_{i}-a^{\prime}+b^{\prime}$ and therefore $y-a+b \sqcap x_{i}-a^{\prime}+b^{\prime}$ is $\left(b \sqcap\left(x_{i}-a^{\prime}+b^{\prime}\right)\right)$.

Case $3 b$ and $b^{\prime}$ are incomparable. Then $y-a+b \sqcap x_{i}-a^{\prime}+b^{\prime}$ is just $b \sqcap b^{\prime}$.

Next, we consider formulae of the form $\psi\left(t(y) \sqcap t^{\prime}(y), t^{\prime \prime}(y, \vec{x})\right)$. In a completely analogous way, we can replace $\psi$ by a formula $\psi^{\prime}$ of the form $\psi^{\prime}(y, \vec{x})=\bigvee_{j} \psi_{j}\left(y, x_{i}\right) \wedge \psi\left(t_{j}\left(y \sqcap t_{j}^{\prime}(y)\right), t^{\prime \prime}(y, \vec{x})\right)$. By Claim 3 , for each $j$, there is a functional $\mathrm{FO}(\mathbf{S})$ formula $\theta_{j}(y, x)$ such that $\theta_{j}\left(s, s \sqcap t_{j}^{\prime}(s)\right)$ holds and such that, for all $r, r^{\prime}$ in $M, \theta_{j}\left(r, r^{\prime}\right)$ holds only if $r^{\prime}=r \sqcap t_{j}^{\prime}(r)$.

Hence, each subformula $\psi\left(t_{j}\left(y \sqcap t_{j}^{\prime}(y)\right), t^{\prime \prime}(y, \vec{x})\right)$ can be replaced by $\psi\left(t_{j}\left(f_{\theta_{j}}(y)\right), t^{\prime \prime}(y, \vec{x})\right)$.

The same reasoning can of course be used to transform formulae $\psi\left(t^{\prime \prime}(y, \vec{x}), t(y) \sqcap t^{\prime}\left(x_{i}\right)\right)$ and $\psi\left(t^{\prime \prime}(y, \vec{x}), t(y) \sqcap\right.$ $\left.t^{\prime}(y)\right)$.

Now we return to the proof of Lemma 3.13. Assume $\left(c^{\prime}, \vec{c}\right) \equiv_{1}\left(d^{\prime}, \vec{d}\right)$. Recall that by Theorem 3.5, if two strings satisfy exactly the same atomic formulae of $\Omega_{\mathbf{S}^{+}}$, then they agree on all $\mathrm{FO}\left(\mathbf{S}^{+}\right)$formulae.

By Claim 5 it is enough to prove that if $\left(c^{\prime}, \vec{c}\right) \equiv_{1}\left(d^{\prime}, \vec{d}\right)$ then $\left(c^{\prime}, \vec{c}\right)$ and $\left(d^{\prime}, \vec{d}\right)$ agree on all atomic $\Omega_{\mathbf{S}_{\text {left }}^{+}}$formulae that have one or two terms of the form $t\left(y \sqcap t^{\prime}\left(x_{i}\right)\right)$ or $t\left(f_{\psi}(y)\right)$, where $t, t^{\prime}$ are nice terms.

Let $\varphi(y, \vec{x})$ be an atomic $\mathbf{S}_{\text {left }}^{+}$formula with two terms, where at least one of the terms is of the form $t\left(y \sqcap t^{\prime}\left(x_{i}\right)\right)$ or $t\left(f_{\psi}(y)\right)$. Assume that $\varphi(y, \vec{x})$ holds for $\left(c^{\prime}, \vec{c}\right)$ (the case where $\varphi(y, \vec{x})$ holds for $\left(d^{\prime}, \vec{d}\right)$ is completely analogous). Let $t(z)=z-a+b$. We distinguish the following cases.

Case 1. One term of $\varphi$ is $t\left(y \sqcap t^{\prime}\left(x_{i}\right)\right)$ or $t\left(f_{\psi}(y)\right)$ and the other does not contain $y$. Hence $\varphi$ is of one of the following forms:

$$
\begin{aligned}
& \text { - } P_{L}\left(t\left(y \sqcap t^{\prime}\left(x_{i}\right)\right), t^{\prime \prime}(\vec{x})\right) \\
& \text { - } P_{L}\left(t^{\prime \prime}(\vec{x}), t\left(y \sqcap t^{\prime}\left(x_{i}\right)\right)\right) \\
& \text { - } P_{L}\left(t\left(f_{\psi}(y)\right), t^{\prime \prime}(\vec{x})\right) \\
& \text { - } P_{L}\left(t^{\prime \prime}(\vec{x}), t\left(f_{\psi}(y)\right)\right)
\end{aligned}
$$

It follows from Claim 2 that in all these subcases one can get rid of the $t$ term, e.g., by adding $-b+a$ to the other term. It is important here that, for a nice term $t_{1}, t_{1}(x) \in L$ is an $\mathrm{FO}(\mathbf{S})$ expressible property. Then the claim follows from the assumption $\left(c^{\prime}, \vec{c}\right) \equiv_{1}\left(d^{\prime}, \vec{d}\right)$. 
Case 2. $\varphi$ is of the form $P_{L}\left(t_{1}\left(y \sqcap t_{2}\left(x_{i}\right)\right), t_{3}\left(y \sqcap t_{4}\left(x_{j}\right)\right)\right)$. By Claim 4 there is an $\mathrm{FO}(\mathbf{S})$ formula $\theta$ such that $\theta\left(c^{\prime}, t_{2}\left(c_{i}\right), t_{4}\left(c_{j}\right)\right)$ holds in $M$ and $\theta\left(r^{\prime}, t_{2}\left(r_{i}\right), t_{4}\left(r_{j}\right)\right)$ implies $P_{L}\left(t_{1}\left(r^{\prime} \sqcap t_{2}\left(r_{i}\right)\right), t_{3}\left(r^{\prime} \sqcap t_{4}\left(r_{j}\right)\right)\right)$, for all $\left(r^{\prime}, \vec{r}\right)$ in $M$. By our assumption $\left(c^{\prime}, \vec{c}\right) \equiv_{1}\left(d^{\prime}, \vec{d}\right)$ it follows that $P_{L}\left(t_{1}\left(y \sqcap t_{2}\left(x_{i}\right)\right), t_{3}\left(y \sqcap t_{4}\left(x_{j}\right)\right)\right)$ holds also for $\left(d^{\prime}, \vec{d}\right)$.

Case 3. $\varphi$ is of the form $P_{L}\left(t\left(y \sqcap t^{\prime}\left(x_{i}\right)\right), t^{\prime \prime}\left(f_{\psi}(y)\right)\right)$ or of the form $P_{L}\left(t^{\prime \prime}\left(f_{\psi}(y)\right) \sqcap t\left(y, t^{\prime}\left(x_{i}\right)\right)\right)$. Again by Claim 2 we can assume that $t^{\prime \prime}$ is empty. Recall that by definition of $\Omega_{\mathbf{S}}$-function terms $f_{\psi}(y) \preceq y$ and therefore $f_{\psi}(y) \sqcap y=f_{\psi}(y)$. Hence, by applying Claim 4 (where we take one term as empty and $s=y$ ) we get a $\mathrm{FO}(\mathbf{S})$ formula $\theta\left(y, t^{\prime}\left(x_{i}\right)\right)$ such that $\theta$ holds for $\left(c^{\prime}, \vec{c}\right)$ and, whenever $\theta\left(y, t^{\prime}\left(x_{i}\right)\right)$ holds for $\left(d^{\prime}, \vec{d}\right)$, then also $\varphi$ holds for $\left(d^{\prime}, \vec{d}\right)$. Again the claim follows from our assumption that $\left(c^{\prime}, \vec{c}\right) \equiv_{1}\left(d^{\prime}, \vec{d}\right)$.

Case 4. Both terms of $\varphi$ are of the form $t\left(f_{\psi}(y)\right)$. In this case, we also get an equivalent $\mathrm{FO}(\mathbf{S})$ formula by first applying Claim 2 to get rid of one symbol $t$ and then applying Claim 4.

This concludes the proof of Lemma 3.13.

Now we come back to the proof of Theorem 3.12. We actually prove the following which is stronger than what is needed for quantifier-elimination.

Lemma 3.14 $\equiv$ has the back-and-forth property in $M$.

As mentioned at the beginning of the proof of the theorem, the statement of the theorem follows from the lemma, as each type of the form $a t p_{\mathbf{S}^{+}}\left(t_{1}\left(c_{i_{1}}\right), \ldots, t_{k}\left(c_{i_{k}}\right)\right)$ is also an atomic type of $\mathbf{S}_{\text {left }}^{+}$.

Let $\vec{c}$ and $\vec{d}$ such that $\vec{c} \equiv \vec{d}$. Our goal is to show, that for each $c^{\prime}$, there is $d^{\prime}$ such that $\left(c^{\prime}, \vec{c}\right) \equiv\left(d^{\prime}, \vec{d}\right)$. By Lemma 3.13 it is enough to find $d^{\prime}$ such that $\left(c^{\prime}, \vec{c}\right) \equiv_{1}\left(d^{\prime}, \vec{d}\right)$.

By compactness, it suffices to show that for all finite sequences $t_{1}, \ldots, t_{k}$ of terms and all sequences $i_{1}, \ldots, i_{k}$ there is a $d^{\prime}$ such that

$$
\operatorname{atp}_{\mathbf{S}^{+}}\left(c^{\prime}, t_{1}\left(c_{i_{1}}\right), \ldots, t_{k}\left(c_{i_{k}}\right)\right)=\operatorname{atp}_{\mathbf{S}^{+}}\left(d^{\prime}, t_{1}\left(d_{i_{1}}\right), \ldots, t_{k}\left(d_{i_{k}}\right)\right)
$$

Let therefore such sequences and $c^{\prime}$ be fixed. Let $T$ be Tree $\left(\left\{t_{j}\left(c_{i_{j}}\right) \mid j \leq k\right\}\right)$. Let $T^{\prime}$ be the corresponding tree for $\vec{d}$. Let $w=\operatorname{Meet}\left(c^{\prime}, T\right), N=\operatorname{Meet}_{+}\left(c^{\prime}, T\right)$ and $P=\operatorname{Meet}_{-}\left(c^{\prime}, T\right)$.

Note that both of these last two strings are given by meets of terms in + and - over $\vec{c}$. Let $N^{\prime}$ be the image of $N$ in the other model (i.e. the corresponding term in $\vec{d}$ ), and $P^{\prime}$ be the image of $P$. Notice that the inductive hypothesis $\vec{c} \equiv \vec{d}$ guarantees that the ordering relation between meets of these terms in $T$ is preserved when we look at the image terms over $\vec{d}$ and $T^{\prime}$. The inductive hypothesis also tells us that $(N, P)$ and $\left(N^{\prime}, P^{\prime}\right)$ are equivalent as string models (that is, models in the usual string signature plus an extra predicate for the shorter string); this is because these terms satisfy all the same atomic formulae of $\mathbf{S}^{+}$, which include all $P_{L} \mathrm{~S}$.

Now let $w^{\prime}$ be between $N^{\prime}$ and $P^{\prime}$ such that the pairs $(N, w)$ and $\left(N^{\prime}, w^{\prime}\right)$, and $(w, P)$ and $\left(w^{\prime}, P^{\prime}\right)$, are elementary equivalent as string models. Such a string $w^{\prime}$ exists because quantifier elimination over $\mathbf{S}^{+}$(Theorem 3.5) implies that $(M, N, P)$ and $\left(M, N^{\prime}, P^{\prime}\right)$ are elementary equivalent in the language of $\mathbf{S}$, and hence for any $w$ there is $w^{\prime}$ such that the equivalence extends to $(M, N, P, w)$ and $\left(M, N, P, w^{\prime}\right)$. It is clear that such a $w^{\prime}$ suffices.

Now, let $d^{\prime}=w^{\prime} \cdot\left(c^{\prime}-w\right)$. We obviously have that $\left(w, c^{\prime}\right)$ and $\left(w^{\prime}, d^{\prime}\right)$ are elementary equivalent as string models. We can now check that $d^{\prime}$ is what we want. We have to show that $\operatorname{Meet}\left(d^{\prime}, T^{\prime}\right)$, Meet $-\left(d^{\prime}, T^{\prime}\right)$ and Meet $+\left(d^{\prime}, T^{\prime}\right)$ are $w^{\prime}, P^{\prime}$ and $N^{\prime}$ respectively, and that for every star-free language $L$ we have: $P_{L}\left(w^{\prime}, d^{\prime}\right)$ iff $P_{L}\left(w, c^{\prime}\right), P_{L}\left(P^{\prime}, w^{\prime}\right)$ iff $P_{L}(P, w)$, and $P_{L}\left(w^{\prime}, N^{\prime}\right)$ iff $P_{L}(w, N)$. All of these easily follow from the definition of $d^{\prime}$.

This finishes the proof of Lemma 3.14 and thus of Theorem 3.12.

From the previous theorem we get the following corollaries. First, the back-and-forth property of $\equiv_{1}$ gives us the following normal form for $\mathrm{FO}\left(\mathbf{S}_{\text {left }}^{+}\right)$formulae.

Corollary 3.15 For every $\mathrm{FO}\left(\mathbf{S}_{\text {left }}\right)$ formula $\rho(x, \vec{y})$ there is an $\mathrm{FO}(\mathbf{S})$ formula $\rho^{\prime}(x, \vec{z})$ and a finite set of nice $\mathbf{S}_{\text {left }}^{+}$ terms $\vec{t}$ such that

$$
\forall x \vec{y}\left(\rho(x, \vec{y}) \leftrightarrow \rho^{\prime}(x, \vec{t}(\vec{y}))\right)
$$

holds in $\mathbf{S}_{\text {left }}$. 
Then Corollary 3.15 for the empty tuple $\vec{y}$ and Corollary 3.7 imply:

Corollary 3.16 Subsets of $\Sigma^{*}$ definable over $\mathbf{S}_{\text {left }}$ are precisely the star-free languages.

For formulae in the language of $\mathbf{S}_{\text {left }}$ (as opposed to $\mathbf{S}_{\text {left }}^{+}$), we can show that bounded quantification suffices, although the notion of bounded quantification is slightly different here from that used in the previous section. Let $N_{p}(s)$ be the prefix-closure of $\left\{s-s_{1}+s_{2}|| s_{1}|,| s_{2} \mid \leq p\right\}$. Clearly $N_{p}(s)$ is definable from $s$ over $\mathbf{S}_{\text {left }}$. We then define $\mathrm{FO}_{*}\left(\mathbf{S}_{\text {left }}\right)$ as the class of $\mathrm{FO}\left(\mathbf{S}_{\text {left }}\right)$ formulae $\varphi(\vec{x})$ in which all quantification is of the form $\exists z \in N_{p}\left(x_{i}\right)$ and $\forall z \in N_{p}\left(x_{i}\right)$, where $x_{i}$ is a free variable of $\varphi$ and $p \geq 0$ arbitrary.

Corollary 3.17 $\mathrm{FO}_{*}\left(\mathbf{S}_{\text {left }}\right)=\mathrm{FO}\left(\mathbf{S}_{\text {left }}\right)$.

Isolation and VC-dimension We now show that the results about isolation and VC-dimension extend from $\mathbf{S}$ to $\mathbf{S}_{\text {left }}$.

Proposition 3.18 $\mathrm{Th}\left(\mathbf{S}_{\mathrm{left}}\right)$ has the isolation property.

Proof. Let $M$ be a model of $\operatorname{Th}\left(\mathbf{S}_{\text {left }}\right), W$ be a pseudo-finite set of elements of $M$, and $a \in M$. Let $p=t p_{M}(a / W)$. We exhibit a countable subset $W_{0}$ of $W$ such that $t p_{M}\left(a / W_{0}\right)$ isolates $t p_{M}(a / W)$.

Let $\vec{e}, \vec{f}$ be finite tuples of finite strings, and let $W(\vec{e}, \vec{f})=\{w-e+f \mid w \in W, e \in \vec{e}, f \in \vec{f}\}$. Let $w_{1}(\vec{e}, \vec{f}), w_{2}(\vec{e}, \vec{f}), w_{3}(\vec{e}, \vec{f}), w_{4}(\vec{e}, \vec{f})$ be elements of $W$ such that for some $e_{1}, e_{2}$ in $\vec{e}$ and some $f_{1}, f_{2} \in \vec{f}$,

$$
\left(w_{1}(\vec{e}, \vec{f})-e_{1}+f_{1}\right) \sqcap\left(w_{2}(\vec{e}, \vec{f})-e_{2}+f_{2}\right)=\operatorname{Meet}_{-}(a, W(\vec{e}, \vec{f}))
$$

and likewise for some $e_{3}, e_{4}, f_{3}, f_{4}$ in $\vec{e}, \vec{f}$

$$
\left(w_{3}(\vec{e}, \vec{f})-e_{3}+f_{3}\right) \sqcap\left(w_{4}(\vec{e}, \vec{f})-e_{4}+f_{4}\right)=\operatorname{Meet}_{+}(a, W(\vec{e}, \vec{f})) .
$$

Take

$$
W_{0}=\bigcup\left\{w_{1}(\vec{e}, \vec{f}), w_{2}(\vec{e}, \vec{f}), w_{3}(\vec{e}, \vec{f}), w_{4}(\vec{e}, \vec{f})\right\},
$$

where the union is taken over all finite tuples of finite strings. Clearly $W_{0}$ is countable. We claim that $t p_{M}\left(a / W_{0}\right)$ isolates $t p_{M}(a / W)$.

Suppose we have $a^{\prime}$ with $t p_{M}\left(a^{\prime} / W_{0}\right)=t p_{M}\left(a / W_{0}\right)$. Note that by construction of $W_{0}$ and definition of $\operatorname{tp}_{M}\left(a / W_{0}\right)$ this implies that $a^{\prime}$ has the same Meet_ and Meet + over each $W(\vec{e}, \vec{f})$ that $a$ does. This also implies that the type of $a^{\prime}-\operatorname{Meet}\left(a^{\prime}, W(\vec{e}, \vec{f})\right)$ is the same as for $a$, and similarly for the type of $\operatorname{Meet}_{+}\left(a^{\prime}, W(\vec{e}, \vec{f})\right)-$ $\operatorname{Meet}\left(a^{\prime}, W(\vec{e}, \vec{f})\right)$ and the type of $\operatorname{Meet}\left(a^{\prime}, W(\vec{e}, \vec{f})\right)$ - Meet $-\left(a^{\prime}, W(\vec{e}, \vec{f})\right)$.

We want to show that $t_{M}\left(a^{\prime} / W\right)=t p_{M}(a / W)$. By quantifier elimination (Theorem 3.12) over $\mathbf{S}_{\text {left }}$, it suffices to show that they have the same atomic types over $\mathbf{S}_{\text {left }}^{+}$.

From the remark above that $a$ and $a^{\prime}$ have the same meets and the same paths between those meets and Meet $_{+}$, Meet - and themselves it follows that whenever an atom of the form $P_{L}\left(t_{1} \sqcap t_{2}, t_{3} \sqcap t_{4}\right)$ holds for $a$, where the $t_{i}$ are either $a$ or nice terms over $\vec{w}$ and where $t_{1} \sqcap t_{2}$ is a direct predecessor of $t_{3} \sqcap t_{4}$ in the tree defined by $W$, then it also holds for $a^{\prime}$. By the normal form for $\mathbf{S}^{+}$queries (Proposition 3.4) we can conclude $a t p_{\mathbf{S}^{+}}(a, \vec{w}-\vec{e}+\vec{f})=$ $a t p_{\mathbf{S}^{+}}\left(a^{\prime}, \vec{w}-\vec{e}+\vec{f}\right)$, for all finite $\vec{e}, \vec{f}$. Hence, by Claim 3.13 we get that $t p_{M}\left(a^{\prime} / W\right)=t p_{M}(a / W)$ have the same atomic types over $\mathbf{S}_{\text {left }}^{+}$, as required.

By Lemma 3.9, we obtain the following.

Corollary 3.19 Every definable family in $\mathbf{S}_{\text {left }}$ has finite VC-dimension. 


\subsection{A regular algebra extending $\mathrm{S}$}

The previous sections presented star-free algebras with attractive properties. We now give an example of a regular algebra that has significantly less expressive power than the rich structure $\mathbf{S}_{\mathrm{len}}$, and which shares some of the nice properties (isolation, finite $\mathrm{VC}, \mathrm{QE}$ ) of the star-free algebras in the previous sections.

This algebra can be obtained by considering two possible ways of extending $\operatorname{FO}(\mathbf{S})$ : the first is by adding the predicates $P_{L}$ for all regular languages $L$; that is, predicates $P_{L}(x, y)$ which hold for $x \preceq y$ such that $y-x \in L$, where $L$ is a regular language. The second extension is by using monadic-second order logic instead of only first-order logic. It turns out that these extensions define exactly the same algebra. We show this, and also show that the resulting regular algebra shares the QE and VC-dimension properties of the star-free algebras defined previously.

Let $\mathbf{S}_{\text {reg }}=\left\langle\Sigma^{*}, \preceq,\left(l_{a}\right)_{a \in \Sigma},\left(P_{L}\right)_{L}\right.$ regular $\rangle$. Since it defines arbitrary regular languages in $\Sigma^{*}$, it is a proper extension of $\mathbf{S}$. Every $\mathrm{FO}\left(\mathbf{S}_{\text {reg }}\right)$-definable set is definable over $\mathbf{S}_{\text {len }}$, because the predicates $P_{L}$ are definable in $\mathbf{S}_{\text {len }}$ (the easiest way to see this is by using the characterization of $\mathbf{S}_{\text {len }}$ definable properties via letter-to-letter automata). Thus, we have:

Proposition 3.20 Subsets of $\Sigma^{*}$ definable over $\mathbf{S}_{\mathrm{reg}}$ are precisely the regular languages.

Let $\mathbf{S}_{\text {reg }}^{+}$be the extension of $\mathbf{S}_{\text {reg }}$ with $\epsilon$ and $\sqcap$. Most of the results about $\mathbf{S}$ and $\mathbf{S}^{+}$from Section 3.2 can be straightforwardly lifted to $\mathbf{S}_{\text {reg }}$ and $\mathbf{S}_{\text {reg. }}^{+}$. For example, the normal form Proposition 3.4 holds for $\mathbf{S}_{\text {reg }}$ if one replaces "star-free" with "regular": the proof given in Section 3.2 applies verbatim. In fact, similar normal form arguments, in a slightly different form, were given in $[52,66]$. We now obtain:

Theorem 3.21 (see [52]) $\mathbf{S}_{\mathrm{reg}}^{+}$admits quantifier elimination.

The normal form result also shows that neither the functions $f_{a}$ nor the predicate el are definable in $\mathbf{S}_{\mathrm{reg}}$ (the latter can also be seen from the fact that $\mathbf{S}_{\text {reg }}$ has QE in a relational signature of bounded arity, and $\mathbf{S}_{\text {len }}$ does not; for inexpressibility of $f_{a}$ it suffices to apply the normal form results to pairs of strings of the form $\left(1 \cdot 0^{k}, 0^{k}\right)$ : since $1 \cdot 0^{k} \sqcap 0^{k}=\epsilon$, it is impossible to check if two sequences of zeros have the same length). One can also show, as in the case of $\mathbf{S}$, that bounded quantification over prefixes is sufficient.

Furthermore, there is a close connection between FO-definability over $\mathbf{S}_{\text {reg }}$ and MSO-definability over $\mathbf{S}$. It was shown in [52] that

$$
\operatorname{MSO}(\mathbf{S})=\operatorname{FO}\left(\mathbf{S}_{\mathrm{reg}}\right) .
$$

This result was used in [52] to show that S2S and WS2S define the same relations over the infinite binary tree. Here S2S refers to the monadic second-order theory of the infinite binary tree, and WS2S to the weak monadic theory (that is, monadic second-order quantification is restricted to finite sets). Note that it follows from [58] that sets, rather than arbitrary relations, definable in S2S and WS2S, are the same.

From the result of [52] it thus follows that the subsets of $\Sigma^{*}$ definable in MSO over $\mathbf{S}_{\text {reg }}$ are precisely the regular languages.

\subsubsection{Automata model, isolation, and VC dimension}

It was proved in [4] that Regular Prefix Relations (RPR) (those definable by Regular Prefix Automata (RPA), introduced in Section 3.2) are exactly those definable in $\operatorname{MSO}(\mathbf{S})$. Thus, the results of [4] and [52] give a characterization of $\mathrm{FO}\left(\mathbf{S}_{\mathrm{reg}}\right)$.

Corollary 3.22 The relations definable in $\mathrm{FO}\left(\mathrm{S}_{\mathrm{reg}}\right)$ are exactly the RPR relations. Thus each relation definable in $\mathrm{FO}\left(\mathbf{S}_{\mathrm{reg}}\right)$ is recognizable by a $R P A$.

The proof of the isolation property for $\mathbf{S}$ (Proposition 3.10) is unaffected by the change from star-free $P_{L}$ to regular $P_{L}$. Thus, we obtain:

Corollary 3.23 $\operatorname{Th}\left(\mathbf{S}_{\mathrm{reg}}\right)$ has the isolation property, and definable families of $\mathbf{S}_{\mathrm{reg}}$ have finite VC-dimension. 


\subsection{A regular algebra extending $\mathrm{S}_{\text {left }}$}

We now give a final example of a regular algebra. Let $\mathbf{S}_{\text {reg,left }}$ be the common expansion of $\mathbf{S}_{\text {left }}$ and $\mathbf{S}_{\text {reg }}$, that is, $\left\langle\Sigma^{*}, \preceq,\left(l_{a}\right)_{a \in \Sigma},\left(f_{a}\right)_{a \in \Sigma},\left(P_{L}\right)_{L}\right.$ regular $\rangle$. Since $\mathbf{S}_{\text {reg }}$ cannot express the functions $f_{a}$, and $\mathbf{S}_{\text {left }}$ cannot define arbitrary regular sets, we see that $\mathbf{S}_{\text {reg,left }}$ is a proper expansion of $\mathbf{S}_{\text {reg }}$ and $\mathbf{S}_{\text {left }}$. Furthermore, all $\mathbf{S}_{\text {reg, left }}$-definable sets are $\mathbf{S}_{\text {len }}$-definable; the finiteness of $\mathrm{VC}$ dimension for $\mathbf{S}_{\text {reg,left }}$, shown below, implies that this containment is proper, too.

Let $\mathbf{S}_{\text {reg,left }}^{+}$be the common expansion of $\mathbf{S}_{\text {left }}^{+}$and $\mathbf{S}_{\text {reg }}$, that is, the expansion of $\mathbf{S}_{\text {reg,left }}$ with $\epsilon$ and $\sqcap$. The techniques of the previous sections can be used to show the following:

Theorem 3.24 $\mathbf{S}_{\mathrm{reg}, \text { left }}^{+}$has quantifier-elimination. Furthermore, $\operatorname{Th}\left(\mathbf{S}_{\mathrm{reg}, \text { left }}\right)$ has the isolation property, and definable families in $\mathbf{S}_{\mathrm{reg} \text {,left }}$ have finite $\mathrm{VC}$-dimension.

Proof. We sketch the proof of QE. This is done by simply mimicking the proof of Theorem 3.12, but with the role of $\mathbf{S}$ played now by $\mathbf{S}_{\text {reg. }}$. Once again, we work in a saturated model $M$, and define the equivalence relations $\equiv$ and $\equiv_{1}$ as in the proof of Theorem 3.12, but the atomic type is with respect to $\mathbf{S}_{\text {reg }}^{+}$. We then show that $\equiv_{1}$ and $\equiv$ are the same. This is done by proving the following modification of Claims 2, 3, 4, and 5, by substituting uniformly $\mathbf{S}_{\text {reg,left }}^{+}$ for $\mathbf{S}_{\text {left }}^{+}$, and $\mathbf{S}_{\text {reg }}$ for $\mathbf{S}$. The property of star-free languages used in each these claims is just that if $L$ is star-free, and $a$ and $b$ are strings, then the set of $x$ such that $x-a+b \in L$ is also star-free. This clearly holds with regular substituted uniformly for star-free.

We then show that $\equiv$ has the back-and-forth property in $M$, which implies QE. The proof is the same as before, but instead of elementary equivalence of string models in first-order logic, we consider their elementary equivalence in monadic second-order logic.

Similarly to $\mathbf{S}_{\text {left }}$, we derive from the proof of Theorem 3.24 the following normal form for $\mathbf{S}_{\text {reg,left }}$ formulae:

Corollary 3.25 For every $\mathrm{FO}\left(\mathbf{S}_{\mathrm{reg}, \mathrm{left}}\right)$ formula $\rho(x, \vec{y})$ there is an $\mathrm{FO}\left(\mathbf{S}_{\mathrm{reg}}\right)$ formula $\rho^{\prime}(x, \vec{z})$ and a finite set of nice $\mathbf{S}_{\text {left }}^{+}$terms $\vec{t}$ such that

$$
\forall x \vec{y} \rho(x, \vec{y}) \leftrightarrow \rho^{\prime}(x, \vec{t}(\vec{y}))
$$

holds in $\mathbf{S}_{\mathrm{reg}, \text { left. }}$

As we have seen earlier that $\mathrm{MSO}(\mathbf{S})=\mathrm{FO}\left(\mathbf{S}_{\mathrm{reg}}\right)$, one might ask if a similar result holds when insertion on the left is allowed; that is, whether $\operatorname{MSO}\left(\mathbf{S}_{\text {left }}\right)=\mathrm{FO}\left(\mathbf{S}_{\text {reg,left }}\right)$. Since the MSO-theory of $\mathbf{S}_{\text {left }}$ is undecidable [67], there is certainly no effective translation. And in fact one can easily see that the two are different. Since the function $g: x \mapsto 0 \cdot x \cdot 1$ is FO-definable in $\mathbf{S}_{\text {left }}$, one can easily see that even weak $\operatorname{MSO}\left(\mathbf{S}_{\text {left }}\right)$, where set quantification is restricted to finite sets, defines $\left\{0^{n} 1^{n} \mid n \geq 0\right\}$, a non-regular set.

We conclude this section with a remark showing that arithmetic properties definable in structures $\mathbf{S}, \mathbf{S}_{\text {left }}, \mathbf{S}_{\text {reg }}, \mathbf{S}_{\text {reg,left }}$ are weaker than those definable in $\mathbf{S}_{\text {len }}$. As we mentioned earlier, under the binary encoding, $\mathbf{S}_{\text {len }}$ gives us an extension of Presburger arithmetic; namely, it defines + and $V_{2}$, where $V_{2}(x)$ is the largest power of 2 that divides $x$. But even $\mathbf{S}_{\text {reg,left }}$ is much weaker:

Proposition 3.26 Neither successor, nor order, nor addition, are definable in $\mathbf{S}_{\mathrm{reg}, \text { left }}$ (and hence in $\mathbf{S}, \mathbf{S}_{\mathrm{reg}}, \mathbf{S}_{\mathrm{left}}$ ).

Proof. Since order is definable from addition, and successor from order, it suffices to show that successor is not definable. Let $x_{k}=10^{k}, y_{k}=1^{k}$; that is, under the binary encoding, $x$ is the successor of $y$. We show that $\left\{\left(x_{k}, y_{k}\right) \mid k>0\right\}$ is not definable in $\mathbf{S}_{\text {reg,left }}$.

Assume it were; by Corollary 3.25 we get a set of nice terms $t_{i}(y)=y-a_{i}+b_{i}$ and a formula $\alpha(x, \vec{z})$ over $\mathbf{S}_{\text {reg }}$ such that $\alpha(x, \vec{t}(y))$ is true iff for some $k, x=x_{k}$ and $y=y_{k}$. For sufficiently large $k, \vec{t}\left(y_{k}\right)$ consists of strings of the form $c_{i} \cdot 1^{k-p_{i}}$ where $c_{i}$ and $p_{i}$ depend on $\vec{t}$ only. As $c_{i} \cdot 1^{k-p_{i}} \cdot 1^{p_{i}}$ is $c_{i} \cdot y_{k}$, there is a formula $\beta\left(x, z_{1}, \ldots, z_{l}\right)$ of $\mathbf{S}_{\text {reg }}$ (where $l$ is the length of $\vec{t}$ ) such that $\beta(x, \vec{z})$ is true iff for some big enough $k, x=x_{k}$ and $z_{i}=c_{i} \cdot y_{k}$.

We now show that for sufficiently large $k$, depending on $\beta$, if $\beta\left(x_{k}, c_{1} y_{k}, \ldots, c_{l} y_{k}\right)$ is true, then for some $m>k$, $\beta\left(x_{m}, c_{1} y_{k}, \ldots, c_{l} y_{k}\right)$ is true. Clearly this will suffice. For this we use the normal form for $\mathbf{S}_{\text {reg }}$ which is analogous 


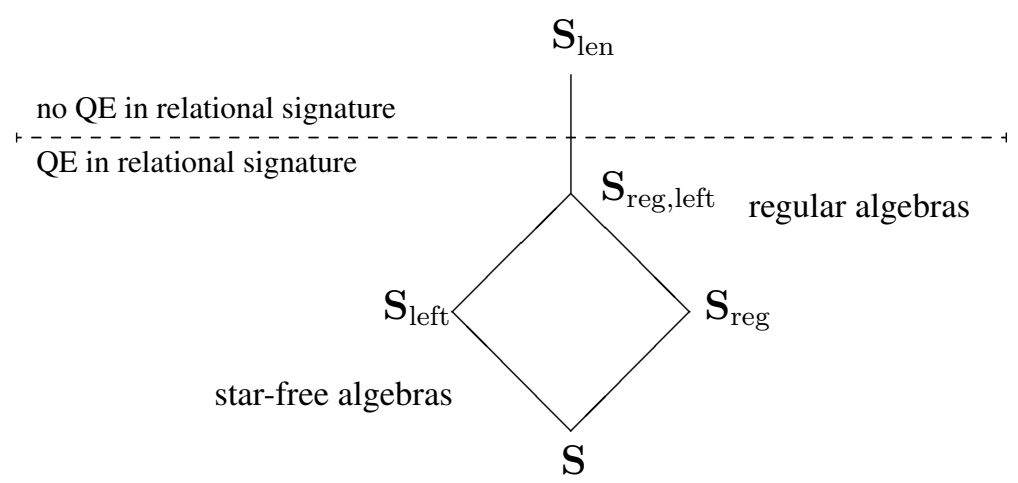

Figure 1: Relationships between $\mathbf{S}, \mathbf{S}_{\text {left }}, \mathbf{S}_{\mathrm{reg}}, \mathbf{S}_{\mathrm{reg}, \text { left }}$, and $\mathbf{S}_{\text {len }}$.

\begin{tabular}{|c|c|l|c|}
\hline Structure & Signature & $\begin{array}{l}\text { Expansion with } \\
\text { quantifier-elimination }\end{array}$ & $\begin{array}{c}\text { Expansion } \\
\text { name }\end{array}$ \\
\hline $\mathbf{S}_{\text {len }}$ & $\prec,\left(l_{a}\right)_{a \in \Sigma}$, el & $\begin{array}{l}\text { all unary relations } \& \\
\text { binary functions }\end{array}$ & $\mathbf{S}_{\text {len }}^{(1,2)}$ \\
\hline $\mathbf{S}$ & $\prec,\left(l_{a}\right)_{a \in \Sigma}$ & $\prec,\left(l_{a}\right)_{a \in \Sigma}, \epsilon, \sqcap$, \\
& $\left(P_{L}\right)_{L \text { star }- \text { free }}$ & $\mathbf{S}^{+}$ \\
\hline $\mathbf{S}_{\text {left }}$ & $\prec,\left(l_{a}\right)_{a \in \Sigma},\left(f_{a}\right)_{a \in \Sigma}$ & $\prec,\left(l_{a}\right)_{a \in \Sigma},\left(f_{a}\right)_{a \in \Sigma}, \epsilon$, & $\mathbf{S}_{\text {left }}^{+}$ \\
& $\prec,\left(l_{a}\right)_{a \in \Sigma},\left(P_{L}\right)_{L}$ regular & $\prec,\left(l_{a}\right)_{a \in \Sigma}, \sqcap,\left(P_{L}\right)_{L \text { star }- \text { free }}, \sqcap,\left(P_{L}\right)_{L \text { regular }}$ & $\mathbf{S}_{\text {reg }}^{+}$ \\
\hline $\mathbf{S}_{\text {reg }}$ & $\prec,\left(l_{a}\right)_{a \in \Sigma},\left(f_{a}\right)_{a \in \Sigma},\left(P_{L}\right)_{L \text { regular }}$ & $\prec,\left(l_{a}\right)_{a \in \Sigma},\left(f_{a}\right)_{a \in \Sigma}, \epsilon$, & $\mathbf{S}_{\text {reg,left }}^{+}$ \\
\hline $\mathbf{S}_{\text {reg,left }}$ & & $(x-a)_{a \in \Sigma}, \sqcap,\left(P_{L}\right)_{L \text { regular }}$ & \\
\hline
\end{tabular}

Definition of $P_{L}: \quad(x, y) \in P_{L}$ iff $x \prec y$ and $y-x \in L$.

Table 1: Summary of quantifier-elimination results

to Proposition 3.4 except that $L$ in $P_{L}$ could be regular. Note that for sufficiently large $k_{0}$, and any $k, m \geq k_{0}$, $\operatorname{Tree}\left(x_{k}, \vec{c} \cdot y_{k}\right)$ is isomorphic (as a tree) to Tree $\left(x_{m}, \vec{c} \cdot y_{k}\right)$. In particular, the predecessor of $x_{k}$ (and $\left.x_{m}\right)$ in such a tree is its meet with one of $c_{i} \cdot y_{k}$, say $c_{1} \cdot y_{k}$. Such a meet is 1 if $c_{1}=\epsilon$, or a prefix of $c_{1}$ if $c_{1} \neq \epsilon$. Thus, $x_{k}-\left(x_{k} \sqcap c_{1} y_{k}\right)$ is either $x_{k}$ or a string $0^{p}$ for $p \geq k-\left|c_{1}\right|$, with $p$ depending only on $c_{1}$. (The same is true when one replaces $k$ by $m$ ).

Let $P_{L}$ be the formula describing the segment $\left(x \sqcap c_{1} y, x\right)$ in the normal form for $\beta$ (we may assume w.l.o.g. that there is only one such formula; if there are several, one can combine them into one by taking the intersection of the languages). Pick $k_{1}, k_{2}>k_{0}$ such that $x_{k_{1}}-\left(c_{1} y_{k_{1}} \sqcap x_{k_{1}}\right)$ is in $L$ iff $x_{k_{2}}-\left(c_{1} y_{k_{1}} \sqcap x_{k_{2}}\right)$ is. It follows from the description of those meets given above that such $k_{1}, k_{2}$ always exist. Now it is immediate from the normal form result that $\beta\left(x_{k_{1}}, c_{1} y_{k_{1}}, \ldots, c_{l} y_{k_{1}}\right)$ iff $\beta\left(x_{k_{2}}, c_{1} y_{k_{1}}, \ldots, c_{l} y_{k_{1}}\right)$, which finishes the proof.

Figure 1 and Table 1 summarize the results of this section.

\section{String query languages}

The goal of this section is to study relational calculi based on the five structures considered in the previous section. Note, however, that most of the previous research on string query languages used concatenation as the main string operation. We give a few simple results indicating that our main goals of getting a low complexity language with an adequate notion of relational algebra cannot be achieved if we include concatenation as a primitive. After that, we explain how operations used in $\mathbf{S}, \mathbf{S}_{\text {left }}, \mathbf{S}_{\text {reg }}, \mathbf{S}_{\text {reg,left }}, \mathbf{S}_{\text {len }}$ are related to SQL string operations, and present properties of relational calculi based on these structures. Most of these are based on model-theoretic properties of the five structures established in Section 3. 


\subsection{Problematic concatenation}

Most earlier papers considered relational calculus with concatenation $\mathrm{RC}_{\text {concat }}$, that is, $\operatorname{RC}\left(S C,\left\langle\Sigma^{*}, \Omega\right\rangle\right)$ where $\Omega$ has the operation of concatenation, and constant symbols for each $a \in \Sigma$. This language is extremely attractive in terms of compositionality: given queries $Q$ and $Q^{\prime}$ returning sets of strings, one can substitute $Q$ and $Q^{\prime}$ within regular-expressions to form new LIKE queries. However, as noticed in [40], for $\Sigma=\{0,1, \sharp\}, \mathrm{RC}_{\text {concat }}$ expresses all computable queries on databases containing strings from $\{0,1\}^{*}$ (see [61] for a proof). In fact, it is easy to show a somewhat stronger result which only requires two letters in $\Sigma$.

Proposition 4.1 Let $\Sigma$ contain at least two letters. Then $\mathrm{RC}_{\text {concat }}$ expresses all computable queries on databases over $\Sigma^{*}$.

Proof. We first show that all computable predicates on $\{0,1\}^{*}$ are expressible. We follow the lines of [61], Chapter III, Theorem 12.4, which uses an extra symbol $\sharp$ to encode a Turing machine computation in $\mathrm{RC}_{\text {concat }}$. Let $M$ be a Turing machine. Let $Q=\left\{q_{2}, \cdots, q_{m}\right\}$ be the set of states of $M, q_{2}$ being the initial state. At step $i$ of the execution of $M$ over an input $x$, the configuration of $M$ can be represented by a string $u_{i} \sharp^{\alpha_{i}} v_{i}, u_{i}, v_{i} \in\{0,1\}^{*}$, where $u_{i}$ is the tape content left of the head, $v_{i}$ is the content of the current position and the positions right of the current position, and $q_{\alpha_{i}}$ is the current state. Let $\varphi_{M}(x)$ be the formula of $\operatorname{RC}_{\text {concat }}$ which states the existence of a string $w \in\{0,1, \sharp\}^{*}$ which will represent the computation of $M$ on $x$. This is done as follows:

1. $w=\sharp^{\alpha_{0}} v_{0} \sharp u_{1} \sharp^{\alpha_{1}} v_{1} \sharp \cdots \sharp u_{n} \sharp^{\alpha_{n}} v_{n}$, for some $n$, where $u_{i}, v_{i} \in\{0,1\}^{*}$.

2. $v_{0}=x, \alpha_{0}=2$.

3. if $u_{i} \sharp^{\alpha} v_{i} \sharp u_{i+1} \sharp^{\beta} v_{i+1}$ is a substring of $w$ then $u_{i+1} \sharp^{\beta} v_{i+1}$ represents the configuration after executing $M$, for one step, from the configuration represented by $u_{i} \sharp^{\alpha} v_{i}$.

4. $q_{\alpha_{n}}$ is an accepting state of $M$.

All the points enumerated above can be checked in $\mathrm{RC}_{\text {concat }}$ [61]. It is also easy to see that the existence of such a string $w$ is equivalent to the acceptance of $x$ by $M$.

In order to remove the extra symbol $\sharp$, the formula $\varphi_{M}(x)$ also states the existence of a string $x_{\sharp}$ of the form $10^{k} 1$, such that none of the strings $u_{i}, v_{i}$ contains $0^{k}$ as a substring. As the computation is finite, such a string always exists and it can easily be distinguished from the $u_{i}$ and $v_{i}$. The formula then states the existence of a string $w^{\prime}$ of the form $x_{\sharp}^{\alpha_{0}} v_{0} x_{\sharp} u_{1} x_{\sharp}^{\alpha_{1}} v_{1} x_{\sharp} \cdots x_{\sharp} u_{n} x_{\sharp}^{\alpha_{n}} v_{n}$ and condition 3 is changed analogously.

Since all computable predicates on $\{0,1\}^{*}$ are expressible, there is a one-to-one mapping $f: \mathbb{N} \rightarrow\{0,1\}^{*}$ such that the image of addition and multiplication under $f$ is expressible in FO over $\left\langle\Sigma^{*}, \cdot, 0,1\right\rangle$. It is known (see [50], Chapter 3), that relational calculus over $\langle\mathbb{N},+, \cdot\rangle$ expresses all computable queries over finite databases (simply by coding finite databases with numbers). Hence, the same coding will apply to $\mathrm{RC}_{\text {concat }}$, showing that it expresses all computable queries.

In databases, we are accustomed to relational calculus having limited expressiveness; then the queries can be analyzed and often good optimizations can be discovered. This is certainly not the case here; moreover, there is no hope of finding a syntax for safe queries.

Corollary 4.2 Let $\Sigma$ contain at least two letters. Then there is no effective syntax for safe queries in $\mathrm{RC}_{\text {concat }}$. Furthermore, the state-safety problem is undecidable for $\mathrm{RC}_{\text {concat }}$.

Proof. This follows from [64]. Indeed from Proposition 4.1, $\mathrm{RC}_{\text {concat }}$ is Turing-complete and thus the structure of [64] in which there is no safe syntax for safe queries, and in which state-safety is undecidable is definable.

Note that when $\Sigma$ has one symbol, $\left\langle\Sigma^{*}, \cdot\right\rangle$ is essentially $\langle\mathbb{N},+\rangle$, and there exists effective syntax for safe queries, and state-safety is decidable [64]. 


\subsection{Basic string operations in SQL}

When looking at existing SQL string operations, the most often-used operation is LIKE pattern-matching. It allows one to say, for example, that a given string is a prefix of another string and also that a string has a fixed string as a substring. LIKE patterns are built from alphabet letters, and characters $\%$ (which matches any string, including $\epsilon$ ), and _ (which matches a single letter). For example, the pattern ab_c $\%$ matches any string whose first letter is $a$, second is $b$, and fourth is $c$. Matching with LIKE can be expressed in first-order logic over S: indeed, with LIKE one can only define star-free languages, which are FO-definable in $\mathbf{S}$.

Another important SQL string operation is the lexicographic ordering $\leq_{\text {lex }}$, which, as we saw earlier, is also expressible in $\mathbf{S}$.

SQL also allows trimming/adding symbols on both left and right of a string. We know that trimming/adding symbols on the right (operation $l_{a}$ and its inverse) is expressible over $\mathbf{S}$, but adding/trimming on the left (operation $f_{a}$ and its inverse) is not. This motivated the study of the structure $\mathbf{S}_{\text {left }}$; it corresponds to LIKE pattern matching, lexicographic ordering, and arbitrary trimming/adding operators of SQL.

The operator LIKE checks membership in a star-free language. The new SQL standard [41] introduces an arbitrary regular expression pattern-matching by a new operator called SIMILAR. Adding this operator corresponds to going from $\mathbf{S}$ to $\mathbf{S}_{\text {reg }}$ or $\mathbf{S}_{\text {left }}$ to $\mathbf{S}_{\text {reg,left }}$ : in both cases, the addition means that the one-dimensional definable families become regular instead of star-free.

Finally, SQL has a string-length operation called LEN. Since this does not return a string, we turn it into a pure string operation that compares lengths of strings: $\mathrm{el}(x, y)$ is true if $|x|=|y|$. Thus, $\mathbf{S}_{\text {len }}$ corresponds to a set of SQL operations that includes LIKE, lexicographic ordering and length comparison. Furthermore, since $\mathbf{S}_{\text {len }}$ subsumes $\mathbf{S}_{\text {left }}, \mathbf{S}_{\text {reg }}$ and $\mathbf{S}_{\text {reg,left }}$, the operator SIMILAR and trimming/adding on the left are expressible over $\mathbf{S}_{\text {len }}$.

\subsection{Expressive power and complexity}

In this section we study expressiveness and complexity of the five relational calculi. We obtain a number of collapse results using the isolation property shown in the first part of the paper, and establish complexity bounds, both in the cases with and without collapse.

\subsubsection{Relational calculus over $S$}

Our goal here is to get bounds on the expressiveness and data complexity for queries in $\mathrm{RC}(\mathbf{S})$. The main tool used is a collapse result, Theorem 4.3, in the spirit of those produced for constraint databases [10,8]. Recall that relational calculus over a domain $\mathrm{RC}(\mathcal{M})$ admits restricted quantifier collapse if every $\operatorname{RC}(S C, \mathcal{M})$ formula $\varphi(\vec{x})$ is equivalent to a formula $\varphi^{\prime}(\vec{x})$ in which $S C$-predicates occur only within the scope of active domain quantifiers $\exists x \in$ adom and $\forall x \in$ adom. It admits the natural-active collapse if every formula is equivalent to one with only active-domain quantifiers.

We already mentioned that the isolation property implies restricted quantifier collapse [8, 32]. From the QE of $\mathbf{S}^{+}$ we also get

Theorem 4.3 $\mathrm{RC}(\mathbf{S})$ admits restricted quantifier collapse, and $\mathrm{RC}\left(\mathbf{S}^{+}\right)$admits the natural-active collapse.

Another quantifier-restriction result is given in the following corollary. Extend $\operatorname{RC}(S C, \mathbf{S})$ with quantifiers of the form $\exists x \prec a d o m$ and $\forall x \prec a d o m$, whose meaning is as follows. Given a formula $\varphi(x, \vec{y})$, an interpretation $\vec{a}$ for $\vec{y}$, and a database $D, \exists x \prec$ adom $\varphi(x, \vec{a})$ states that there exists a string $c$ making $\varphi(c, \vec{a})$ true such that either $c \preceq a_{i}$ for $a_{i}$ a component of $\vec{a}$, or $c \preceq b$ where $b$ is in $\operatorname{adom}(D)$. Since bounded quantification suffices for $\mathbf{S}$ formulae (Corollary 3.6), we obtain:

Corollary 4.4 Every $\operatorname{RC}(S C, \mathbf{S})$ formula is equivalent to a formula that only uses quantifiers $\exists x \prec$ adom and $\forall x \prec$ adom.

We note that a a straightforward corollary of Theorem 4.3 shows that the data complexity for $\mathrm{RC}(\mathbf{S})$ matches that of pure relational calculus. 
Corollary 4.5 The data complexity of $\mathrm{RC}(\mathbf{S})$ is in $\mathrm{AC}^{0}$. In particular, neither parity nor connectivity test is expressible in $\mathrm{RC}(\mathbf{S})$.

Proof. By Corollary 4.4 we can assume that a given query $\varphi(\vec{x})$ is of the form $Q \vec{y} \in$ adom $\bigvee \wedge \alpha_{i}(\vec{x}, \vec{y})$ where each $\alpha_{i}$ is either an atomic or negated atomic $S C$-formula, or an $\mathbf{S}$ formula, in which all quantification is restricted to prefixes of $\vec{x}, \vec{y}$. The proof then follows the standard proof of $\mathrm{AC}^{0}$ data complexity for the relational calculus (see, for example, [1]), and one only has to prove that each $\mathbf{S}$ formula can be evaluated in $\mathrm{AC}^{0}$.

Suppose $\alpha\left(z_{1}, \ldots, z_{k}\right)$ is an $\mathbf{S}$ formula in which all quantification is restricted to prefixes of $z_{i}$ s. With $\vec{z}$, associate a structure $S_{\vec{z}}$ of the signature consisting of unary predicates $Z_{i},\left(P_{a}\right)_{a \in \Sigma}$, \# and a binary predicate $<$ as follows: the domain is $\{1, \ldots, M\}$, where $M=\sum_{i}\left|z_{i}\right|+(k-1)$, and the interpretation of $<$ is standard. The first $\left|z_{1}\right|$ elements belong to $Z_{1}$, followed by an element that belongs to \#, followed by $\left|z_{2}\right|$ elements that belong to $Z_{2}$ etc. The membership in $P_{a}$ is determined by the corresponding symbol in the $z_{i}$ s. To show that $\alpha$ can be evaluated in $\mathrm{AC}^{0}$, it is enough to show that there is a $\mathrm{FO}(\mathrm{BIT},<)$ sentence $\beta$ such that $\mathbf{S} \models \alpha(\vec{z})$ iff $S_{\vec{z}} \models \beta$. This is done by a straightforward induction on the structure of $\alpha$, as one can encode the prefix relation over $S_{\vec{z}}$ using the definability of + and $\times$ in $\mathrm{FO}(\mathrm{BIT},<)($ cf. [47]).

Another corollary concerns the expressive power of generic queries. Recall that a query is generic if it commutes with permutations on the domain; in other words, it is independent of specific elements stored in a database.

Every query expressible in pure relational calculus is generic. Examples of other generic queries are parity test and graph connectivity test; these are well known to be inexpressible in relational calculus.

Combining Theorem 4.3 with the active generic collapse [10], we obtain:

Corollary 4.6 Every generic query expressible in $\mathrm{RC}(\mathbf{S})$ is already expressible in $\mathrm{RC}(<)$, relational calculus over ordered databases.

With respect to time complexity Corollary 4.5 only gives a polynomial upper bound. We show next that for unary databases we get a much stricter complexity result. We call a database schema $S C$ unary if it only contains unary relation names. We next show that queries over unary databases can be evaluated in linear time. This is because a unary database can be transformed into a tree, and a query can be transformed into a first-order sentence over the tree, which can then be evaluated by a tree automaton. More precisely, we have:

Proposition 4.7 Let $S C$ be unary. Then every Boolean $\operatorname{RC}(S C, \mathbf{S})$-query can be evaluated in linear time in the size of the database.

Proof. Let $S C$ be unary. We define a representation of $S C$-databases by finite labeled trees as follows. Let $R_{1}, \ldots, R_{m}$ be the relation names of $S C$. Let, for simplicity, $\Sigma$ be $\{0,1\}$. Let $X=\left\{x_{1}, \ldots, x_{k}\right\}$ be a set of variables. For a finite database $D$ over $S C$ and a vector $\vec{a}=a_{1}, \ldots, a_{k}$ of strings from $\Sigma^{*}$ the (finite) tree $t=t(D, \vec{a})$ is defined as follows.

- The set of vertices of $t$ is $\operatorname{prefix}(D, \vec{a})$.

- Each vertex $v$ of $t$ is labeled by a $0-1$-vector $\vec{r}(v)=\left(r_{1}(v), \ldots, r_{m}(v)\right)$, where $r_{i}(v)=1$ if and only if $v \in R_{i}$.

- Each vertex $v$ is labeled by a subset $X(v)$ of $X$, where $x_{i} \in X(v)$ if and only if $a_{i}=v$.

It should be pointed out that all leaves $v$ of $t(D, \vec{a})$ carry a label $\vec{r}(v)$ with at least one non-zero entry or a label $X(v)$ which is not the empty set.

It is straightforward that, for each $\mathrm{RC}(S C, \mathbf{S})$-formula $\varphi$ (with prefix quantification) there is a first-order formula $\varphi^{\prime}$ on labeled trees (represented as finite structures in the usual way) such that for each $S C$-database $D$ and each vector $\vec{a}=a_{1}, \ldots, a_{k}$ of strings, $(D, \vec{a}) \models \varphi$ if and only if $t(D, \vec{a}) \models \varphi^{\prime}$. In the case of Boolean queries, $k$ equals 0 . As it is well-known that even MSO-sentences can be evaluated in linear time on labeled trees (e.g., via the simulation of suitable tree automata, see [67]), we can conclude the desired complexity bound.

From Lemma 4.20 below and the results of [56] it follows that safe unary $\operatorname{RC}(S C, \mathbf{S})$-queries (i.e., with one free variable) can be evaluated in linear time in the size of the database. By combining this with the techniques of [62] it can be shown that, in general, $k$-ary queries can be evaluated in time $O\left(n^{k}\right)$ for databases of size $n$. 


\subsubsection{Relational calculus over $S_{\text {len }}$}

We have seen that query evaluation for relational calculus over $\mathbf{S}$ has low complexity. However, many useful queries of low complexity, such as the query that appends a fixed string on the left of a given column, are not expressible in $\mathbf{S}$. Hence we examine the addition of the equal length predicate, that is, relational calculus over $\mathbf{S}_{\text {len }}$. Throughout this section, we again assume that the alphabet has at least two symbols (as over the one-symbol alphabet, equal length is simply equality and thus does not give us any extra power).

To analyze the expressive power and complexity of $\mathbf{S}_{\text {len }}$, we again make use of a normal-form result for queries. In this case it is no longer sufficient to quantify over prefixes of strings in the active domain; however a different restricted quantification suffices.

We introduce quantifiers $\exists|x| \leq$ adom and $\forall|x| \leq$ adom to be interpreted as follows. Given a formula $\varphi(\vec{y})$, a database $D$ and an interpretation $\vec{a}$ for $\vec{y}$, a subformula $\exists|x| \leq$ adom $\alpha(x, \cdot)$ is satisfied if there exists a string $c$ satisfying $\alpha(c, \cdot)$ such that the length of $c$ does not exceed the length of the longest string in $\operatorname{adom}(D)$ and $\vec{a}$. We call these length-restricted quantifiers. Note that they are just a notational convenience, as they can be expressed in $\mathrm{RC}\left(\mathbf{S}_{\text {len }}\right)$. Moreover, they capture the expressiveness of $\mathrm{RC}\left(\mathbf{S}_{\text {len }}\right)$ :

Proposition 4.8 Every $\mathrm{RC}\left(S C, \mathbf{S}_{\mathrm{len}}\right)$ formula is equivalent to a formula that uses only length-restricted quantifiers.

Proof. For an $S C$-database $D$ and a tuple of strings $\vec{s}$, we use the notation $\downarrow(D, \vec{s})$ for $\left\{s^{\prime} \mid \exists s \in \operatorname{adom}(D) \cup \vec{s}\right.$ : $\left.\left|s^{\prime}\right| \leq|s|\right\}$, and $S[D, \vec{s}]$ for the structure with the universe $\downarrow(D, \vec{s})$ in the language of $\mathbf{S}_{\text {len }}$ plus the $S C$-relations, plus constants for the elements of $\vec{s}$. We write $\mathbf{S}_{\text {len }}(D, \vec{s})$ for the structure in the same language whose universe is $\Sigma^{*}$. Let $m$ be the maximum arity of any relation name of $S C$.

We write $\left(D_{1}, \vec{s}_{1}\right) \equiv_{k}\left(D_{2}, \vec{s}_{2}\right)$ if the duplicator has a winning strategy in the $k$-round Ehrenfeucht-Fraïssé game on $\mathbf{S}_{\text {len }}\left(D_{1}, \vec{s}_{1}\right)$ and $\mathbf{S}_{\text {len }}\left(D_{2}, \vec{s}_{2}\right)$, and $\left(D_{1}, \vec{s}_{1}\right) \equiv_{k}^{b}\left(D_{2}, \vec{s}_{2}\right)$ if the duplicator has a winning strategy in the $k$-round Ehrenfeucht-Fraïssé game on $S\left[D_{1}, \vec{s}_{1}\right]$ and $S\left[D_{2}, \vec{s}_{2}\right]$. We claim that $\equiv_{k+m+1}^{b}$ refines $\equiv_{k}$. By the Ehrenfeucht-Fraïssé theorem (cf. [27, 47]), this implies the result, as both equivalence relations are of finite index, each class of $\equiv_{k+m+1}^{b}$ is definable with length-restricted quantifiers, and each $\operatorname{RC}\left(\mathbf{S}_{\text {len }}\right)$ query of quantifier rank $k$ is a union of $\equiv_{k}$-classes.

We now describe the winning strategy for the duplicator for $k$ moves in the game on $\mathbf{S}_{\text {len }}\left(D_{1}, \vec{s}_{1}\right)$ and $\mathbf{S}_{\text {len }}\left(D_{2}, \vec{s}_{2}\right)$. Let $l_{j}$ be the maximum length of a string in $S\left[D_{j}, \vec{s}_{j}\right], j=1,2$. In response to each move, say $a_{i} \in \mathbf{S}_{\text {len }}\left(D_{1}, \vec{s}_{1}\right)$ by the spoiler, the duplicator produces, in addition to his response $b_{i} \in \mathbf{S}_{\text {len }}\left(D_{2}, \vec{s}_{2}\right)$, two extra elements $a_{i}^{\prime} \in S\left[D_{1}, \vec{s}_{1}\right]$ and $b_{i}^{\prime} \in S\left[D_{2}, \vec{s}_{2}\right]$. This is done as follows. Suppose the rounds $1, \ldots, i-1$ have already been played, and the spoiler plays $a_{i} \in \mathbf{S}_{\text {len }}\left(D_{1}, \vec{s}_{1}\right)$.

There are two cases. If $a_{i} \in S\left[D_{1}, \vec{s}_{1}\right]$, then $a_{i}^{\prime}=a_{i}$, and the duplicator looks at the position $\left(a_{1}^{\prime}, \ldots, a_{i-1}^{\prime}, a_{i}^{\prime}\right),\left(b_{1}^{\prime}, \ldots, b_{i-1}^{\prime}\right)$ in the game on $S\left[D_{1}, \vec{s}_{1}\right]$ and $S\left[D_{2}, \vec{s}_{2}\right]$, and selects $b_{i}^{\prime} \in S\left[D_{2}, \vec{s}_{2}\right]$ according to his winning strategy. He then sets $b_{i}=b_{i}^{\prime}$.

In the other case, we have $a_{i} \notin S\left[D_{1}, \vec{s}_{1}\right]$, that is, $\left|a_{i}\right|>l_{1}$. Let $a_{i}^{\prime}$ be the prefix of $a_{i}$ of length $l_{1}$. As before, the duplicator now looks at the configuration $\left(a_{1}^{\prime}, \ldots, a_{i-1}^{\prime}, a_{i}^{\prime}\right),\left(b_{1}^{\prime}, \ldots, b_{i-1}^{\prime}\right)$ in the game on $S\left[D_{1}, \vec{s}_{1}\right]$ and $S\left[D_{2}, \vec{s}_{2}\right]$, and selects $b_{i}^{\prime}$ as the response to $a_{i}^{\prime}$. Note that $b_{i}^{\prime}$ is of length $l_{2}$. Indeed, since the duplicator can play in the game on $S\left[D_{1}, \vec{s}_{1}\right]$ and $S\left[D_{2}, \vec{s}_{2}\right]$ for $k+m+1$ moves, for every move up to $k$ his response to a string of length $l_{1}$ must be a string of length $l_{2}$, for otherwise with the next $m+1$ moves the spoiler would be able to choose an extension $b_{i+1}$ of $b_{i}$ and strings $b_{i+2}, \ldots, b_{i+m+1}$ such that $b_{i+2}$ has the same length as $b_{i+1}$ and is in $\vec{s}_{2}$ or $D_{2}$. The latter might be witnessed by the strings $b_{i+3}, \ldots, b_{i+m+1}$. The duplicator would have no suitable response in $S\left[D_{1}, \vec{s}_{1}\right]$. We now set $b_{i}=b_{i}^{\prime} \cdot x$, where $x=a_{i}-a_{i}^{\prime}$, that is, $x$ is the relative suffix of $a_{i}^{\prime}$ in $a_{i}$. It follows immediately that this strategy ensures the win by the duplicator in the $k$-round game on $\mathbf{S}_{\text {len }}\left(D_{1}, \vec{s}_{1}\right)$ and $\mathbf{S}_{\text {len }}\left(D_{2}, \vec{s}_{2}\right)$.

Prefix-restricted quantification does not suffice for $\operatorname{RC}\left(\mathbf{S}_{\text {len }}\right)$. Indeed, consider the following query $Q$ on a unary relation $U: Q(U)$ is true iff $U$ contains a single element, which is from $0^{*}$ and of even length. This is expressible in $\mathrm{RC}\left(\mathbf{S}_{\text {len }}\right)$ by

$$
\exists ! x U(x) \wedge \forall x\left(U(x) \rightarrow\left(x \in 0^{*}\right) \wedge \exists z \in(01)^{*} \operatorname{el}(z, x)\right),
$$

where $\exists ! x U(x)$ expresses that there is exactly one $x$ with $U(x)$. Note that the predicates $x \in 0^{*}$ and $z \in(01)^{*}$ can be expressed even over $\mathbf{S}$ : recall that $\mathbf{S}$ can define any star-free language and $\mathbf{S}_{\text {len }}$ any regular language. However, this query $Q$ is inexpressible with just prefix quantification: if it were, then over single-element databases contained in $0^{*}$, the predicate el could be replaced by equality. Hence the set of strings from $0^{*}$ of even length would be definable over 
S. But this language is not star-free, and this contradicts the fact that the languages definable over $\mathbf{S}$ are exactly the star-free languages (Corollary 3.7).

As with Theorem 4.3, from Proposition 4.8 we get us a rough upper bound on the complexity of $\mathrm{RC}\left(\mathbf{S}_{\text {len }}\right)$, which should be compared with Corollary 4.11 and Proposition 4.12 below:

Corollary 4.9 The data complexity of $\mathrm{RC}\left(\mathbf{S}_{\mathrm{len}}\right)$ is in $\mathrm{PH}$.

Proof. To check if $D \models \varphi(\vec{a})$, it is enough to quantify over strings whose length does not exceed $N$, where $N$ is the maximum length of a string in $\operatorname{adom}(D) \cup \vec{a}$ (see Proposition 4.8). If $\varphi$ has alternation depth $k$ this can be done by a polynomial time alternating Turing machine with $k$ alternations, hence in $\mathrm{PH}$.

The result below establishes two bounds. The first one is for complexity of generic queries in $\operatorname{RC}\left(\mathbf{S}_{\text {len }}\right)$. That is, the complexity of the language $\{\operatorname{enc}(D) \# \operatorname{enc}(\mathrm{t}) \mid D \models \varphi(t)\}$ for a generic $\varphi$. The other complexity bound is very useful for proving expressibility results. A relational (Boolean) query is a set of isomorphism types of $S C$-databases (w.r.t. the $S C$-relations only). A relational query is in $\mathrm{AC}^{0}$ if it is in $\mathrm{AC}^{0}$ under the usual relational encoding enc $c_{0}$ : elements of a $k$-element active domain are encoded by $1, \ldots, k$, in binary (cf. [1]). A relational query $Q$ is expressible in $\operatorname{RC}\left(\mathbf{S}_{\text {len }}\right)$ if there is a $\operatorname{RC}\left(\mathbf{S}_{\text {len }}\right)$ sentence $\Phi$ such that the $S C$-isomorphism type of $D$ is in $Q$ iff $D \models \Phi$.

Theorem 4.10 The data complexity of generic queries in $\mathrm{RC}\left(\mathrm{S}_{\mathrm{len}}\right)$ is in $\mathrm{TC}^{0}$. Furthermore, any relational query that is expressible in $\mathrm{RC}\left(\mathbf{S}_{\text {len }}\right)$ is in $\mathrm{AC}^{0}$.

Proof. Without loss of generality, we consider Boolean queries and assume that $\Sigma=\{0,1\}$. For a string $s \in \Sigma^{*}$, let $N(s)$ be the number which is $1 \cdot s$ in binary. Let $s<_{N} s^{\prime}$ iff $N(s)<N\left(s^{\prime}\right)$. Note that for strings of length $k, N(s)$ ranges from $2^{k}$ to $2^{k+1}-1$, and $|s|<\left|s^{\prime}\right|$ implies $N(s)<N\left(s^{\prime}\right)$. We call a database nice if the set $\{N(s) \mid s \in \operatorname{adom}(D)\}$ is of the form $\{1, \ldots, n\}$ for some $n \geq 1$. Note that the maximum length of a string in such a database is $l(n)=\left\lceil\log _{2}(n+1)\right\rceil-1$.

Now we claim that every Boolean generic query $\Phi$ can be evaluated in $\mathrm{AC}^{0}$ over nice databases. By Proposition 4.8 , without loss of generality, all quantifiers in $\Phi$ are assumed to be length-restricted. With a nice database $D$, we associate a new database $D^{\prime}$ of the same schema with the universe $\{1, \ldots, n\}=\{N(s) \mid s \in \operatorname{adom}(D)\}$, such that $\left(t_{1}, \ldots, t_{k}\right) \in R$ in $D$ iff $\left(N\left(t_{1}\right), \ldots, N\left(t_{k}\right)\right) \in R$ in $D^{\prime}$. We next show that $\Phi$ can be expressed in FO(BIT, $<$ ) over structures of the form $D^{\prime}$, where $D$ is nice. This will suffice to prove the claim, as the encodings of $D$ and $D^{\prime}$ are identical, and $\mathrm{FO}(\mathrm{BIT},<)$ captures uniform $\mathrm{AC}^{0}$ [7]. Recall the definition of BIT from Section 2. We also recall that the usual arithmetic predicates ( + and $\times$, given as ternary predicates) are definable in $\mathrm{FO}(\mathrm{BIT},<$ ), and so are many other helpful predicates, for example, a predicate for the powers of 2 [47].

There are two main problems: first, quantification in $\Phi$ is restricted to the maximum length of a string (that is, over nice databases, quantifiers in $\Phi$ range not over $\{1, \ldots, n\}$ but rather $\left.\left\{1, \ldots, 2^{l(n)+1}-1\right\}\right)$; second, we must show that the operations of $\mathbf{S}_{\text {len }}$ can be expressed.

To deal with the first problem, we assume that $\Phi$ is in prenex form, and replace each quantifier $\exists s$ with two quantifiers $\exists i_{s} \exists i_{s}^{\prime}$. Each string $s$ of length not exceeding $l(n)$ can be represented uniquely by two numbers $i_{s}, i_{s}^{\prime}$ such that:

$$
i_{s}=\left\{\begin{array}{rl}
N(s) & \text { if } N(s) \leq n, \\
n & \text { if } N(s)>n,
\end{array} \quad i_{s}^{\prime}=\left\{\begin{aligned}
2^{l(n)} & \text { if } N(s) \leq n, \\
N(s)-n & \text { if } N(s)>n .
\end{aligned}\right.\right.
$$

Note that $i_{s}, i_{s}^{\prime} \leq n$, and for $N(s)>n, i_{s}^{\prime}<2^{l(n)}$, if $|s| \leq l(n)$. For each new pair of quantifiers $\exists i_{s} \exists i_{s}^{\prime}$ we add a formula stating that $i_{s}, i_{s}^{\prime}$ satisfy the following conditions: either $i_{s}<n$ and $i_{s}^{\prime}=2^{l(n)}$, or $i_{s}=n$, and $i_{s}^{\prime}<2^{l(n)}$. This can be done in $\mathrm{FO}(\mathrm{BIT},<)$, as the condition $x=2^{l(n)}$ is expressible (it says that $x$ is the largest power of 2 that does not exceed $n$, which is expressible with BIT).

Next, we must show how to translate the atomic and negated atomic subformulae of $\Phi$. Each subformula of the form $R\left(s_{1}, \ldots, s_{k}\right)$, where $R \in S C$, is translated into $R\left(i_{s_{1}}, \ldots, i_{s_{k}}\right) \wedge \bigwedge_{i} i_{s_{i}}^{\prime}=2^{l(n)}$. Checking $L_{0}(s)$ is simply $\neg \operatorname{BIT}\left(i_{s}, 1\right)$, and $L_{1}(s)$ is $\operatorname{BIT}\left(i_{s}, 1\right)$. For el $(s, u)$, one has to check that the largest power of 2 not exceeding $i_{s}+i_{s}^{\prime}$ and $i_{u}+i_{u}^{\prime}$ is the same. This happens iff either both $i_{s}^{\prime}, i_{u}^{\prime}$ are less than $2^{l(n)}$ (in this case $|s|=|u|=l(n)$ ), or both equal $2^{l(n)}$ (in which case both $s$ and $u$ are in the active domain), and for each $p \leq \max \left(i_{s}, i_{u}\right)$ which is a power of 2 , $p \theta i_{s} \leftrightarrow p \theta i_{u}$, where $\theta$ ranges over the comparisons $<,>$ and $=$. These conditions can be expressed in FO(BIT, $\left.<\right)$. 
We now consider the predicate $s \prec u$. There are four cases. If both $i_{s}^{\prime}, i_{u}^{\prime}<2^{l(n)}$, this is false, as $s, u$ are not in the active domain, and hence of the same length. Similarly if $i_{s}^{\prime}<2^{l(n)}$ and $i_{u}^{\prime}=2^{l(n)}$, then $s \prec u$ is false.

The third case is when $i_{s}^{\prime}=i_{u}^{\prime}=2^{l(n)}$. In this case both $s$ and $u$ are in the active domain, and the formula below states that $s \prec u$ :

$$
\begin{aligned}
\exists p, p^{\prime} \quad & \operatorname{FirstBIT}\left(i_{s}, p\right) \wedge \operatorname{FirstBIT}\left(i_{u}, p^{\prime}\right) \wedge p<p^{\prime} \wedge \\
& \forall q \leq p^{\prime} \operatorname{BIT}\left(i_{s}, p-q\right) \leftrightarrow \operatorname{BIT}\left(i_{u}, p^{\prime}-q\right),
\end{aligned}
$$

where $\operatorname{FirstBIT}(u, p)$ is the formula

$$
\operatorname{BIT}(u, p) \wedge \forall q(p<q \leq l(n)) \rightarrow \neg \operatorname{BIT}(u, q)
$$

expressing that $u$ has length $p$.

The last case is when $i_{s}^{\prime}=2^{l(n)}$ and $i_{u}^{\prime}<2^{l(n)}$ (that is, $s$ is in the active domain, $u$ is not). We reduce it to the previous case as follows: $s \prec u$ iff $s=v$ or $s \prec v$, where $v$ is the immediate predecessor (in the $\prec$ relation) of $u$. Note that for $u$ of length $l(n)$, its predecessor is in the active domain, so if we can state this condition, then the previous case applies to test if $s \prec v$. To check that a number $m$ is such that $v$ with $N(v)=m$ is an immediate predecessor of $u$, we consider two subcases. In the first subcase, $n+i_{u}^{\prime}$ is odd (this can be tested with BIT). In that case, one should test if $2 m+1=n+i_{u}^{\prime}$. Note that in $\mathrm{FO}(\mathrm{BIT},<)$ we can only quantify over numbers not exceeding $n$, so this test is done by

$$
\exists k(k+m=n) \wedge\left(k+i_{u}^{\prime}=m+1\right) .
$$

In the subcase when $n+i_{u}^{\prime}$ is even, one should test if $2 m=n+i_{u}^{\prime}$, which is done by $\exists k(k+m=n) \wedge\left(k+i_{u}^{\prime}=m\right)$.

Thus, we have shown that every Boolean query can be evaluated in $\mathrm{AC}^{0}$ over nice databases. Now let $Q$ be a Boolean relational query $Q$, that is expressible in $\operatorname{RC}(C)$ by a query $\Psi$. There is a family of circuits $\mathcal{C}$ that computes $\Psi$ on nice databases. Now, for a relational database, let $e n c_{0}(D)$ be the standard encoding under which elements of the active domain of size $k$ are coded as integers $1, \ldots, k$ in binary. Given an arbitrary relational database $D$, consider $e n c_{0}(D)$ as the input to $\mathcal{C}$. Let $D_{0}$ be a (nice) database over strings obtained from $D$ by replacing the $i$ th element of the active domain with the string $s$ such that $N(s)=i$. Then $\operatorname{enc}\left(D_{0}\right)=e n c_{0}(D)$, and thus when it is given to $\mathcal{C}, \mathcal{C}$ returns $\Psi\left(D_{0}\right)$. But by genericity, we have $Q(D)=Q\left(D_{0}\right)=\Psi\left(D_{0}\right)$, which implies that $Q$ is in $\mathrm{AC}^{0}$.

It remains to show that the data complexity of generic queries in $\operatorname{RC}\left(\mathbf{S}_{\text {len }}\right)$ is in $\mathrm{TC}^{0}$. Let $\Psi$ be a generic query definable in $\mathrm{RC}\left(\mathbf{S}_{\text {len }}\right)$. For each database $D$, let nice $(D)$ be a database obtained from $D$ as follows: let $\operatorname{adom}(D)=$ $\left\{s_{1}, \ldots, s_{k}\right\}$, where $s_{1} \leq_{\text {lex }} \ldots \leq_{\text {lex }} s_{k}$. Then in nice $(D)$, each $s_{i}$ from $D$ is replaced by a string $s_{i}^{\prime}$ with $N\left(s_{i}^{\prime}\right)=i$. Note that this transformation can be carried out in $\mathrm{TC}^{0}$, as $\leq_{\text {lex }}$ is in $\mathrm{AC}^{0}$ by Corollary 4.5, and counting the number of elements satisfying a formula can be done in $\mathrm{TC}^{0}$ [7]. Furthermore, by genericity, $D \models \Psi$ iff nice $(D) \models \Psi$. The latter can be checked in $\mathrm{AC}^{0}$, which gives us a $\mathrm{TC}^{0}$ upper bound on the data complexity of generic queries. The theorem is proved.

One cannot draw any definite conclusions from the first statement of Theorem 4.10 , as $\mathrm{TC}^{0}$ is not yet separated from NP (although widely believed to be properly contained in DLogSpace). However, the second statement, and known lower bounds for $\mathrm{AC}^{0}[2,35]$ give us:

Corollary 4.11 Parity test and connectivity test are not definable in $\mathrm{RC}\left(\mathbf{S}_{\mathrm{len}}\right)$.

We now prove lower bounds that show the complexity of $\mathbf{S}_{\text {len }}$ queries, although within $\mathrm{PH}$, may be prohibitively high. Let $\operatorname{MSO}(S C)$ be the class of queries over $S C$ expressible in monadic second-order logic. This includes queries of high-complexity, namely for each level of the polynomial hierarchy, $\mathrm{PH}$, complete queries [3], in particular, NP-complete and coNP-complete ones (3-colorability and its complement). Such queries cannot be expressed over arbitrary databases in $\mathrm{RC}\left(\mathbf{S}_{\text {len }}\right)$ (e.g., not over nice ones); however, they can be expressed under some additional assumptions.

We say that the width of the active domain of an $S C$ database $D$ (over $\Sigma^{*}$ ) is $k$ if $k$ is the maximal size of a subset of $\operatorname{adom}(D)$ whose elements are pairwise incomparable by the prefix relation. It should be noted that every database $D$ can be transformed into a database $D^{\prime}$ of width 1 which is isomorphic to $D$ with respect to the $S C$-predicates.

Proposition 4.12 For every fixed $k$, all $\mathrm{MSO}(S C)$-expressible queries can be expressed over databases of width at most $k$ in $\mathrm{RC}\left(S C, \mathbf{S}_{\text {len }}\right)$. 
Proof. Assume without loss of generality that $0,1 \in \Sigma$. For a database $D$ of width $k$, the set of $\preceq$-maximal elements $\left\{s_{1}, \ldots, s_{l}\right\}$ of $\operatorname{adom}(D)$ has cardinality $l \leq k$, and thus $\operatorname{prefix}(D)$ is the union of chains $\operatorname{prefix}\left(s_{1}\right), \ldots, \operatorname{prefix}\left(s_{l}\right)$, where $\operatorname{prefix}(s)=\left\{s^{\prime} \mid s^{\prime} \preceq s\right\}$. The idea of the proof is this: a subset $Z$ of $\operatorname{prefix}(s)$ can be modeled by a string $s_{Z} \in\{0,1\}^{*}$ of the same length as $s$, such that $s^{\prime} \preceq s$ is in $Z$ iff the prefix of $s_{Z}$ of the length $\left|s^{\prime}\right|$ ends on a 1 .

Now suppose an $\operatorname{MSO}(S C)$ query $Q$ is given. We assume it is expressed by an MSO sentence $\Phi$ in which all quantified second-order variables are distinct. Let $m_{1}, \ldots, m_{k}$ be fresh first-order variables (to be interpreted as maximal elements of $\operatorname{adom}(D))$. We then associate with each second-order quantifier $\exists Z$ new first-order variables $s_{Z}^{1}, \ldots, s_{Z}^{k}$, and define the following transformation $\varphi \mapsto \varphi^{\circ}$ of subformulae of $\Phi$ :

- Every atomic subformula other than $Z(x)$, where $Z$ is a second-order variable, is unchanged.

- Every subformula $Z(x)$ is replaced by $(Z(x))^{\circ}$ defined as

$$
\bigvee_{i=1}^{k} x \preceq m_{i} \wedge \exists y \preceq s_{Z}^{i} \operatorname{el}(y, x) \wedge L_{1}(y) .
$$

- $\left(\varphi_{1} * \varphi_{2}\right)^{\circ}=\varphi_{1}^{\circ} * \varphi_{2}^{\circ}$, where $*$ is $\wedge$ or $\vee,(\neg \varphi)^{\circ}=\neg \varphi^{\circ},(\exists u \varphi)^{\circ}=\exists u \varphi^{\circ}$, where $u$ is a first-order variable.

- A subformula $\exists Z \varphi$ is replaced by $(\exists Z \varphi)^{\circ}$ defined as

$$
\exists s_{Z}^{1}, \ldots, s_{Z}^{k} \bigwedge_{i=1}^{k} \operatorname{el}\left(s_{Z}^{i}, m_{i}\right) \wedge \varphi^{\circ} .
$$

The result of this transformation is an open $\mathrm{RC}\left(\mathbf{S}_{\text {len }}\right)$ query $\Phi^{\circ}\left(m_{1}, \ldots, m_{k}\right)$. We now define a Boolean $\operatorname{RC}\left(\mathbf{S}_{\text {len }}\right)$ as

$$
\exists m_{1} \in \operatorname{adom} \ldots \exists m_{k} \in \text { adom } \forall u \in \operatorname{adom} \bigvee_{i} u \preceq m_{i} \wedge \Phi^{\circ}\left(m_{1}, \ldots, m_{k}\right),
$$

stating that $m_{1}, \ldots, m_{k}$ list all (not necessarily distinct) maximal elements of $\operatorname{adom}(D)$, and that $\Phi^{\circ}\left(m_{1}, \ldots, m_{k}\right)$ holds. For a database of width at most $k$, this means that $\Phi^{\circ}\left(m_{1}, \ldots, m_{k}\right)$ holds for the list of all maximal elements in $\operatorname{adom}(D)$, which happens iff $D \models \Phi$.

Thus, while not computationally complete as $\mathrm{RC}_{\text {concat }}, \mathrm{RC}\left(\mathbf{S}_{\text {len }}\right)$ can express some queries that normally would not be expected to be expressible in a first-order language.

Recall that we had a linear time bound for the evaluation of Boolean $\mathrm{RC}(\mathbf{S})$-queries on unary databases. We show next, that this might not be the case for $\mathrm{RC}\left(\mathbf{S}_{\text {len }}\right)$. Even worse, there might be even no fixed polynomial bound.

We consider ordered graphs as finite structures with a universe $U$ of the form $\{1, \ldots, n\}$, the natural order relation $<$ on $U$ and a binary relation $E$. Let $S C$ be the database schema with one unary relation name $R$.

Lemma 4.13 For every first-order formula $\varphi$ on ordered graphs there is a $\mathrm{RC}\left(S C, \mathbf{S}_{\text {len }}\right)$-formula $\varphi^{\prime}$ and an algorithm which computes for each graph $G$ an $S C$-database $D_{G}$ such that $G \models \varphi$ if and only if $D_{G} \models \varphi^{\prime}$. Furthermore, the algorithm works in time $O\left(n^{2} \log n\right)$ on graphs with $n$ vertices and the maximum length of a string in $D_{G}$ is $2\left\lceil\log _{2} n\right\rceil+1$ and, consequently, the size of $D_{G}$ is $O\left(n^{2} \log n\right)$.

Proof. We give the proof for $\Sigma=\{0,1\}$. Let an ordered graph $G$ with $n$ vertices be given and let $m:=\left\lceil\log _{2} n\right\rceil$. We define $D_{G}$ as follows. Let $a_{1}, \ldots, a_{n}$ denote the lexicographically first $n$ strings of length $m$. We define the set $R$ as

$$
\left\{a_{1}, \ldots, a_{n}\right\} \cup\left\{a_{i} \cdot 0 \cdot a_{i} \mid i \leq n\right\} \cup\left\{a_{i} \cdot 1 \cdot a_{j} \mid(j, i) \in E\right\}
$$

Intuitively, the strings $a_{1}, \ldots, a_{n}$ represent the vertices of $G$. There is an edge from vertex $j$ to vertex $i$ if and only if $a_{i} \cdot 1 \cdot a_{j} \in R$. The vertices $a_{i} \cdot 0 \cdot a_{i}$ are used to get $a_{i}$ from $a_{j} \cdot 1 \cdot a_{i}$.

It is straightforward to check that $D_{G}$ has the desired size and can be produced in time $O\left(n^{2} \log n\right)$ assuming a suitable representation of $G$. 
The formula $\varphi^{\prime}$ is obtained from $\varphi$ as follows. First, all subformulas of the form $\exists x \psi(x)$ are replaced by $\exists x \in$ $\operatorname{adom}(\neg \exists y$ y $\prec x \wedge R(y)) \wedge \psi(x)$. Intuitively, the quantification is restricted to minimal elements of the active domain of $D_{G}$, i.e., to $a_{1}, \ldots, a_{n}$. Note however that the next two steps will introduce new unrestricted quantifiers.

Next, atomic formulas $x<y$ are replaced by

$$
\exists z, z_{0}, z_{1} l_{0}(z)=z_{0} \wedge l_{1}(z)=z_{1} \wedge z_{0} \preceq x \wedge z_{1} \preceq y
$$

Finally, atomic formulas $E(x, y)$ are replaced by

$$
\begin{aligned}
& \exists x_{1}, x_{2}, y_{1}, y_{2} \quad l_{0}(x)=x_{1} \wedge l_{1}(y)=y_{1} \wedge x_{1} \preceq x_{2} \wedge y_{1} \preceq y_{2} \wedge R\left(x_{2}\right) \wedge R\left(y_{2}\right) \wedge \\
& \forall x_{3}, y_{3}\left(x_{1} \prec x_{3} \preceq x_{2} \wedge y_{1} \prec y_{3} \preceq y_{2} \wedge \operatorname{el}\left(x_{3}, y_{3}\right)\right) \rightarrow\left(L_{0}\left(x_{3}\right) \leftrightarrow L_{0}\left(y_{3}\right)\right)
\end{aligned}
$$

which states the existence of strings $x_{2}$ and $y_{2}$ of the form $x 0 x$ and $y 1 x^{\prime}$ and such that, second line of the formula, $x=x^{\prime}$. It is straightforward to check that $G \models \varphi$ if and only if $D_{G} \models \varphi^{\prime}$.

It follows from the lemma that a linear (or fixed polynomial) bound for the evaluation of Boolean $\operatorname{RC}\left(\mathbf{S}_{\text {len }}\right)$ queries on unary databases would imply a fixed polynomial bound for the data complexity of first-order sentences on ordered graphs. It would imply further a fixed polynomial bound for the evaluation of first-order sentences on BIT-structures (cf., [6]). This, in turn, would separate first-order logic from least fixed point logic on such structures and therefore imply the validity of the ordered conjecture [49] with various consequences in complexity theory (see [6] for a discussion).

We cannot conclude from this connection that linear time evaluation for $\mathrm{RC}\left(\mathbf{S}_{\text {len }}\right)$ queries on unary databases is impossible. But we cannot expect a proof as simple as that of Proposition 4.7 for $\mathrm{RC}(\mathbf{S})$.

\subsubsection{Relational calculi over $S_{\text {left }}, S_{\text {reg }}$ and $S_{\text {reg,left }}$}

These calculi behave similarly to $\operatorname{RC}(\mathbf{S})$, although some complexity bounds are slightly different. From the isolation property shown for all the structures and from QE results we conclude the following:

Theorem 4.14 $\mathrm{RC}\left(\mathbf{S}_{\text {left }}\right), \mathrm{RC}\left(\mathbf{S}_{\mathrm{reg}}\right)$, and $\mathrm{RC}\left(\mathbf{S}_{\mathrm{reg}, \text { left }}\right)$ admit the restricted quantifier collapse. Furthermore, $\mathrm{RC}\left(\mathbf{S}_{\text {left }}^{+}\right), \operatorname{RC}\left(\mathbf{S}_{\mathrm{reg}}^{+}\right)$, and $\mathrm{RC}\left(\mathbf{S}_{\text {reg,left }}^{+}\right)$admit the natural-active collapse.

Corollary 4.15 $\mathrm{RC}\left(\mathbf{S}_{\text {left }}\right)$ queries have $\mathrm{AC}^{0}$ data complexity, while $\mathrm{RC}\left(\mathbf{S}_{\mathrm{reg}}\right)$ and $\mathrm{RC}\left(\mathbf{S}_{\mathrm{reg}, \text { left }}\right)$ queries have $\mathrm{NC}{ }^{1}$ data complexity. Furthermore, every generic query expressible in $\mathrm{RC}\left(\mathbf{S}_{\mathrm{left}}\right)$ or $\mathrm{RC}\left(\mathbf{S}_{\mathrm{reg}}\right)$ is expressible in $\mathrm{RC}(<)$.

Proof. The proof of the $\mathrm{AC}^{0}$ bound is the same as for Corollary 4.5 except that we need to show that each fixed $\mathbf{S}_{\text {left }}$ formula can be evaluated in $\mathrm{AC}^{0}$. By the quantifier elimination result quoted in the proof of Theorem 4.14, it suffices to show that every fixed quantifier-free formula in $\mathbf{S}_{\text {left }}^{+}$can be evaluated in $\mathrm{AC}^{0}$. For that, we notice that every $\mathbf{S}_{\text {left }}^{+}$ term can be evaluated in $\mathrm{AC}^{0}$ (since both $x-a$ and $a \cdot x$ operations are available), and the rest follows the proof for $\mathbf{S}$.

For $\mathbf{S}_{\text {reg, }}$, we again use the collapse result and the proof that $\mathrm{RC}(\mathbf{S})$ queries with active-domain quantification can be evaluated in $\mathrm{AC}^{0}$ (and hence $\mathrm{NC}^{1}$ ). The only difference is in evaluating the $P_{L}$ predicates, which can no longer be done in $\mathrm{AC}^{0}$ as $L$ may not be star-free. However, every regular language is in $\mathrm{NC}^{1}$ [65], and thus $P_{L}$ can be evaluated in $\mathrm{NC}^{1}$ on its inputs, showing that the data complexity of $\mathrm{RC}\left(\mathbf{S}_{\mathrm{reg}}\right)$ is in $\mathrm{NC}^{1}$. The proof for $\mathbf{S}_{\mathrm{reg} \text {, left }}$ combines the proofs for $\mathbf{S}_{\text {left }}$ and $\mathbf{S}_{\text {reg. }}$.

The last statement follows from the collapse result and [10].

Note the contrast of the above with Proposition 4.12, which implies that relational calculus over $\mathbf{S}_{\text {len }}$ contains problems complete for each level of the polynomial hierarchy. Theorem 4.14 is the key for obtaining low data complexity. It follows from the isolation property of the underlying structure, which fails for $\mathbf{S}_{\text {len }}$ as it does not have finite VC-dimension (recall Proposition 3.2). 


\subsection{Safe Queries}

All the relational calculi we study here contain queries that sometimes produce infinite output. Thus one of our goals is to syntactically capture the safe queries in these languages, and to be able to analyze safety properties of a query for example, given an arbitrary query and a database, to tell whether the output of the query on that database is finite. We saw that this cannot be done if the set of operations includes concatenation. In contrast, for our five structures, we can syntactically describe safe queries, give an algebra that captures these queries, and extend the major decidability results for query safety analysis that hold for pure relational calculus.

\subsubsection{Effective syntax for safe queries: defining finiteness}

The simplest way to show that safe queries in $\operatorname{RC}(\mathcal{M})$ have effective syntax is to show that one can test if a given query returns a finite result on a given database. To do so, it is enough to ensure that finiteness is definable in $\mathrm{RC}(\mathcal{M})$. Formally, finiteness is definable in $\operatorname{RC}(\mathcal{M})$ if there exists a sentence $\Phi^{\text {safe }}$ in the language of $\mathcal{M}$ and $S C$ expanded with a single new unary predicate symbol $U$ such that for any query $\varphi(x)$ and any database $D,(D, \varphi(D)) \models \Phi^{\text {safe }}$ iff $\varphi(D)$ is finite. For example, finiteness is easily definable in $\mathrm{RC}\left(\mathbf{S}_{\text {len }}\right)$ by

$$
\exists y \forall x(U(x) \rightarrow \exists z \prec y \operatorname{el}(z, x)) .
$$

Once finiteness is definable, an enumeration of safe queries can easily be obtained. Given a query $\varphi(\vec{x})$, let $\psi_{\varphi}(x)$

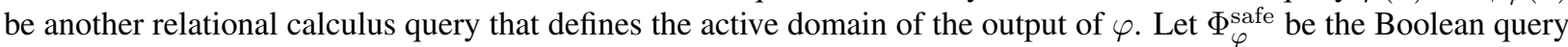
obtained from $\Phi^{\text {safe }}$ be replacing $U(\cdot)$ by $\psi_{\varphi}(\cdot)$. Then $\varphi(\vec{x}) \wedge \Phi_{\varphi}^{\text {safe }}$ lists all safe queries.

For traditional relational calculus, and for its analogs over order constraints, linear constraints, and polynomial constraints, finiteness can easily be shown to be definable [11]. It is thus surprising that for $\mathrm{RC}(\mathbf{S})$ this approach does not work:

Proposition 4.16 Finiteness is not definable in $\mathrm{RC}(\mathbf{S})$.

Proof. We prove the proposition for $\Sigma=\{0,1\}$; it is straightforward to generalize this for any alphabet. We consider databases with one unary predicate $U$. We show by an Ehrenfeucht-Fraïssé game argument that, for each $k$, there are databases $A_{k}$ and $B_{k}$ such that $U$ is a finite set in $A_{k}$ and an infinite set in $B_{k}$ but $A_{k}$ and $B_{k}$ can not be distinguished by a $\mathrm{RC}(\mathbf{S})$-formula of quantifier rank $k$.

Let $k \geq 0$ be fixed.

Let $T_{i}$ denote the set of strings of length at most $i$. Intuitively, $T_{i}$ is the full binary tree of depth $i$ (and formally it is the same as $\Sigma \leq i$ ).

We use $\equiv_{k}$ to denote equivalence in the $k$-round Ehrenfeucht game on structures based on $\mathbf{S}$ and $\equiv_{k}^{s}$ to denote equivalence in the $k$-round Ehrenfeucht-Fraïssé game on strings.

We will use the following Claim.

Claim 1 There exist $N$ and $n>0$ (depending on $k$ ) such that for each $i \geq N$ it holds that $\left(\mathbf{S}, T_{i}\right) \equiv_{k}\left(\mathbf{S}, T_{i+n}\right)$. Without loss of generality we can choose $N$ as a multiple of $n$.

Proof of the Claim: For every $k, \equiv_{k}$ has finitely many equivalence class. Let $N$ be this number. By the pigeon-hole principle there exists two integers $i, j$ such that $i \leq N+1$ and $j \leq N+1$ and $T_{i} \equiv_{k} T_{j}$. We show that for any two integers $u, v, T_{u} \equiv T_{v}$ implies $T_{u+1} \equiv T_{v+1}$, the claim will then follow with $n=j-i$. To prove the latter notice that $T_{u+1}$ is simply $|\Sigma|$ copies of $T_{u}$ plus one node. Similarly $T_{v+1}$ is simply $|\Sigma|$ copies of $T_{v}$ plus one node. The FO $\mathrm{F}_{k}$ strategy on $T_{u+1}$ and $T_{v+1}$ mimics the strategy for $T_{u}$ and $T_{v}$ on each copy separately and the root is played as soon as the other root is played.

Let $m=2^{k} n$ and $M=2^{3 k} k n+N$. Let $A_{k}$ be $\left(\mathbf{S}, T_{M}\right)$.

Next, we define an infinite set $S$ such that $A_{k}$ and $(\mathbf{S}, S)$ can not be distinguished by a formula of depth $k$. Let $h$ be the string homomorphism which maps 0 to $0^{m}$ and 1 to $1^{m}$. We call a string $w$ normal if it is of the form $h\left((01)^{i}\right)$, for some $i \geq 0$. We call $w$ semi-normal if it is $h(v)$ for some string $v$. The set $S$ is defined as the set of all strings of 
the form $u v$, where $u$ is a normal string and $v$ is a string of length at most $N+2 m$. We set $B_{k}=(\mathbf{S}, S)$. Note that $S$ is prefix-closed and that all maximal strings in $B_{k}$ have a length which is a multiple of $n$.

For two strings $u$ and $w$ such that $u$ is a prefix of $w$ we write $A_{k}[u, w]$ for the substructure of $A_{k}$ that consists of all strings $v$ such that $u$ is a prefix of $v$ but $w$ is not a strict prefix of $v$ and analogously for $B_{k}$. Let $\operatorname{Mod}_{n}$ denote a sequence $Z_{0}, \ldots, Z_{n-1}$ of unary relations over (initial segments of) the natural numbers such that $Z_{i}(j)$ holds if and only if $(j \bmod n)=i$.

For later use we need the following lemma.

Lemma 4.17 (a) Let $v, w$ be semi-normal strings and $v^{\prime}, w^{\prime}$ normal strings such that $v$ is a prefix of $w$ and $v^{\prime}$ is a prefix of $w^{\prime}$ and $|w| \leq M-N$. Let $u=w-v$ and $u^{\prime}=w^{\prime}-v^{\prime}$. If $\left(u, \operatorname{Mod}_{n}\right) \equiv_{k}^{s}\left(u^{\prime}, \operatorname{Mod}_{n}\right)$ then $A_{k}[v, w] \equiv_{k} B_{k}\left[v^{\prime}, w^{\prime}\right]$.

(b) $\left(h(0), \operatorname{Mod}_{n}\right) \equiv_{k}^{s}\left(h(00), \operatorname{Mod}_{n}\right)$ and $\left(h(01), \operatorname{Mod}_{n}\right) \equiv_{k}^{s}\left(h(001), \operatorname{Mod}_{n}\right)$.

(c) For each $i \geq 2^{k}+1$ it holds that $\left(h\left((01)^{2^{k}+1}\right), \operatorname{Mod}_{n}\right) \equiv_{k}^{s}\left(h\left((01)^{i}\right), \operatorname{Mod}_{n}\right)$.

Proof of Lemma 4.17.

(a) Intuitively in the tree $T_{M},[v, w]$ consists of the path from $v$ to $w$ and of trees branching off the strings on that path. By definition of $A_{k}$ the tree branching off a string $z$ of the path has depth $M-|z|-1$ which is at least $N$ and congruent to $N-|z-v|-1$ modulo $n$, as $M, N$ and $|v|$ are multiples of $n$. More precisely, we refer here to the tree that is rooted at the child of $z$ which is not a prefix of $w$. Analogously, if $z^{\prime}$ is a string of the path from $v^{\prime}$ to $w^{\prime}$ in $B_{k}$ there is a tree of depth $(2 m+N)-\left|z^{\prime}-y^{\prime}\right|-1$ branching off $z^{\prime}$, where $y^{\prime}$ is the longest normal string which is a prefix of $z^{\prime}$. Hence, the depth of this tree is at least $N$ and it is congruent to $N-\left|z^{\prime}-v^{\prime}\right|-1$ modulo $n$. We can conclude from Claim 1 that the branching trees at $z$ and $z^{\prime}$ are $k$-equivalent, whenever $|z-v|$ and $\left|z^{\prime}-v^{\prime}\right|$ are congruent modulo $n$.

By combining the winning strategy of the duplicator on $\left(u, \operatorname{Mod}_{n}\right)$ and $\left(u^{\prime}, \operatorname{Mod}_{n}\right)$ with the winning strategies on the off-branching trees we get (a).

(b) The first statement is shown by a standard game argument using the fact that $h(0)$ is the concatenation of $2^{k}$ strings of length $n$. Each of these substrings is identically labeled by $\operatorname{Mod}_{n}$. In a $k$ round game this can not be distinguished from the concatenation of $2 \cdot 2^{k}$ such strings. The second statement follows directly from the first one.

(c) This can also be shown by a standard argument.

Next, we have to show that $\left(\mathbf{S}, T_{M}\right) \equiv_{k}(\mathbf{S}, S)$.

Claim 2 The duplicator can play the $k$ round Ehrenfeucht-Fraïssé game in a way that guarantees that the following holds after $l$ rounds of the game.

Let $\vec{a}=a_{1}, \ldots, a_{l}$ denote the selected elements of $A_{k}$ and let $\vec{b}=b_{1}, \ldots, b_{l}$ denote the corresponding elements in $B_{k}$.

There is a semi-normal string $p_{l}$ and a normal string $q_{l}$ (the pivot strings) such that

1. None of the $a_{i}$ has $p_{l}$ as a prefix and none of the $b_{i}$ has $q_{l}$ as a prefix.

2. $\left(A_{k}-p_{l} *, \vec{a}\right) \equiv_{k}\left(B_{k}-q_{l} *, \vec{b}\right)$.

3. $\left|p_{l}\right| \leq l 2^{3 k} n$.

Here, $A_{k}-p_{l} *$ denotes the substructure of $A_{k}$ in which all strings that have $p_{l}$ as a strict prefix are omitted and in which $p_{l}$ is a distinguished constant (and analogously for $B_{k}-q_{l} *$ ).

Proof of the claim. It should be noted that, as $q_{l}$ is normal, $B_{k}-q_{l} *$ only contains a finite part of $S$. In the proof, it will always be the case that $p_{l}$ is a prefix of $p_{l+1}$ and $q_{l}$ is a prefix of $q_{l+1}$. 
Because of condition (1) we can conclude from (2) that there is a partial $\mathbf{S}$-isomorphism from $\vec{a}$ ) to $\vec{b}$ ) at the end of the game. Hence the claim implies the statement of the theorem.

We prove the claim by induction on $l$. For $l=0$ we choose $p_{0}=q_{0}=\epsilon$. This guarantees (1)-(3).

Now assume that, for some $l<k, l$ rounds have been played and there are $p_{l}$ and $q_{l}$ such that (1)-(3) hold. We show that the duplicator can play in a way such that, for suitable choices of $p_{l+1}$ and $q_{l+1}(1)-(3)$ also holds for $l+1$.

We distinguish 3 cases.

Case 1. The spoiler chooses a vertex in $A_{k}-p_{l} *$ or $B_{k}-q_{l} *$. Then we simply set $p_{l+1}=p_{l}$ and $q_{l+1}=q_{l}$ and (1)-(3) follow directly.

Case 2. The spoiler chooses a string $a_{l+1}$ which has $p_{l}$ as a prefix. Let $u=a_{l+1}-p_{l}$.

- If $u$ is of the form $h(01) \cdot v$, for some $v$ then we set $p_{l+1}=p_{l} \cdot h(001)$ and $q_{l+1}=q_{l} \cdot h(01)$.

- Otherwise we set $p_{l+1}=p_{l} \cdot h(01)$ and $q_{l+1}=q_{l} \cdot h(01)$.

In both subcases, $p_{l+1}$ is not a prefix of $a_{l+1}$. As $\left|p_{l+1}\right| \leq\left|p_{l}\right|+3 m \leq M-N$ it follows from Lemma 4.17 (a) and (b) that in both subcases $A_{k}\left[p_{l}, p_{l+1}\right] \equiv_{k} B_{k}\left[q_{l}, q_{l+1}\right]$. Therefore the duplicator can choose a string $b_{l+1}$ in $B_{k}\left[q_{l}, q_{l+1}\right]$ that guarantees a winning strategy on $A_{k}\left[p_{l}, p_{l+1}\right]$ and $B_{k}\left[q_{l}, q_{l+1}\right]$ for $k-1$ more rounds. By combining this winning strategy with the winning strategy on $\left(A_{k}-p_{l} *, \vec{a}\right)$ and $\left(B_{k}-q_{l} *, \vec{b}\right)$ we obtain a $k-l-1$ round winning strategy on $\left(A_{k}-p_{l+1} *, \vec{a}, a_{l+1}\right)$ and $\left(B_{k}-q_{l+1} *, \vec{b}, b_{l+1}\right)$. Hence, we can conclude (2). Furthermore, of course, (1) and (3) hold.

Case 3. The spoiler chooses a string $b_{l+1}$ which has $q_{l}$ as a prefix. Let $i$ be maximal such that $b_{l+1}$ can be written as $q_{l} \cdot h\left((01)^{i}\right) \cdot v$, for some string $v$. We choose $q_{l+1}=q_{l} \cdot h\left((01)^{i+1}\right)$ and

$$
p_{l+1}=\left\{\begin{array}{cl}
p_{l} \cdot h\left((01)^{i+1}\right) & \text { if } i \leq 2^{k}, \\
p_{l} \cdot h\left((01)^{2^{k}+1}\right) & \text { otherwise. }
\end{array}\right.
$$

The choice of $q_{l+1}$ guarantees that it is not a prefix of $b_{l+1}$. From Lemma 4.17 (c) and (a) it follows that in both subcases $A_{k}\left[p_{l}, p_{l+1}\right] \equiv_{k} B_{k}\left[q_{l}, q_{l+1}\right]$. This implies the existence of an appropriate $a_{l+1}$ in $A_{k}\left[p_{l}, p_{l+1}\right]$ such that (2) holds again. By the choice of $p_{l+1}$ and induction we also get (1) and (3).

This completes the proof of the proposition.

\subsubsection{Effective syntax for safe queries: range-restriction}

While post-checking finiteness is a way to obtain effective syntax for safe queries, one often wishes to have a more explicit representation of safe queries. It turns out that we can get natural representations for safe queries in $\mathrm{RC}(\mathbf{S})$ and $\mathrm{RC}\left(\mathbf{S}_{\text {len }}\right)$ and other calculi. The technique we use derives from work on safe languages with linear or polynomial constraints [11]: for each query $Q$, we effectively construct another safe query $Q^{\prime}$ that gives an upper bound on $Q(D)$, if it is finite. Such explicit constructions are used to prove the theorem below, as well as to provide relational algebra extensions.

We follow the idea of range-restriction as presented in [11]. A formula $\gamma(x, z)$ over $\mathcal{M}$ is called algebraic if for every $b$, the set $\{a \mid \mathcal{M} \models \gamma(a, b)\}$ is finite. An $\operatorname{RC}(\mathcal{M})$ query in range-restricted form is a pair $Q=\left(\gamma(x, y), \varphi\left(x_{1}, \ldots, x_{n}\right)\right)$, where $\varphi$ is an arbitrary query and $\gamma$ is an algebraic formula over $\mathcal{M}$. The semantics is given by $\varphi(\vec{x}) \wedge \exists \vec{y} \in \operatorname{adom}\left(\bigwedge_{i} \gamma\left(x_{i}, y_{i}\right)\right)$. That is,

$$
Q(D)=\gamma(\operatorname{adom}(D))^{n} \cap \varphi(D)
$$

where $\gamma(X)=\{a \mid \gamma(a, b)$ for some $b \in X\}$. Clearly, every query in range-restricted form is safe.

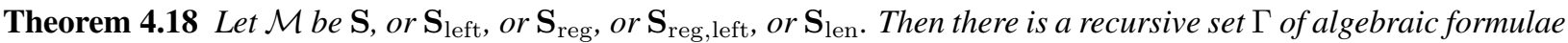
over $\mathcal{M}$ such that, given a query $\varphi(\vec{x})$ in $\operatorname{RC}(\mathcal{M})$, there is $\gamma(x, y) \in \Gamma$ with the property that the range-restricted query $Q=(\gamma, \varphi)$ coincides with $\varphi$ on all databases over which $\varphi$ is safe. 
Proof. The proof is based on a number of lemmas, which show that if a query $\varphi(x)$ is satisfied by an element that is sufficiently far from $\operatorname{adom}(D)$, then $\varphi$ returns an infinite result on $D$. The definition of "sufficiently far" depends on the particular structure.

First, we need two observations. The first one is a generalized version of the pumping lemma for finite automata.

Lemma 4.19 For each sequence $L_{1}, \ldots, L_{m}$ of regular languages there is a number $k$ such that for each string $z$, $|z|>k$, there are strings $u, v, w$, with $z=u v w$ and $|v|>0$, such that for each string $x$, each $j \in\{1, \ldots, m\}$ and each $i>0$,

$$
x u v w \in L_{j} \Longleftrightarrow x u v^{i} w \in L_{j} .
$$

Proof of Lemma 4.19. Let, for each $i \leq m, A_{i}$ be a deterministic automaton for $L_{i}$ with transition function $\delta_{i}$. Without loss of generality we assume that all automata have the same set $\{1, \ldots, n\}$ of states with 1 as the initial state. Let $k:=n^{n m}$ and $z$ be a string with $|z|>k$. For each $j \leq m, \alpha \leq n$ and $l \leq|z|$, let $q_{j \alpha l}$ be defined as $\delta_{j}(\alpha, z[1, l])$, where $z[1, l]$ is the prefix of $z$ of length $l$. I.e., $q_{j \alpha l}$ is the state of $A_{j}$ after reading the first $l$ symbols of $z$ starting from state $\alpha$. As $|z|>k$ there must be $l_{1} \neq l_{2}$ such that $q_{j \alpha l_{1}}=q_{j \alpha l_{2}}$, for all $j \leq m$ and $\alpha \leq n$. Let $u, v, w$ be chosen such that $z=u v w, u$ is the prefix of $z$ of length $l_{1}$ and $v$ is of length $l_{2}-l_{1}$. We claim that for every $j \leq m$, every $i>0$ and every string $x, x u v w \in L_{j}$ if and only if $x u v^{i} w \in L_{j}$. Indeed, let $\alpha$ be the state $\delta_{j}(1, x)$. Then, as $q_{j \alpha l_{1}}=q_{j \alpha l_{2}}$ we have $\delta_{j}(\alpha, u)=\delta_{j}(\alpha, u v)=\delta_{j}\left(\alpha, u v^{i}\right)$. Therefore $x u v w$ is accepted by $A_{j}$ if and only if $x u v^{i} w$ is accepted by $A_{j}$.

Using this lemma, we show:

Claim. Let $\mathcal{M}=\left\langle\Sigma^{*}, \Omega\right\rangle$ be such that all operations in $\Omega$ are definable in $\mathbf{S}_{\text {len }}$. Then, for every $r>0$, there exists $k>0$ such that for any string $s$ with $|s| \geq k$, there are infinitely many strings $s^{\prime}$ satisfying $(\mathcal{M}, s) \equiv_{r}\left(\mathcal{M}, s^{\prime}\right)$.

Proof of the claim. Indeed, let $\alpha_{1}(x), \ldots, \alpha_{l}(x)$ list formulae (of quantifier rank $r$ ) that define all the $r$-types of a single string over $\mathcal{M}$. Since each $\alpha_{i}$ is definable over $\mathbf{S}_{\text {len }}$, there is a DFA $A_{i}$ which accepts a string $s$ iff $\mathcal{M} \models \alpha_{i}(s)$ [14]. In particular, the set of strings $s$ which make $\alpha_{i}(s)$ true is a regular language $L_{i}$. From Lemma 4.19 it follows, that there is a $k$ such that, for each string $s$ with $|s|>k$ there are infinitely many strings $s^{\prime}$ that are contained exactly in the same languages $L_{i}$ as $s$, i.e., make the same formulas $\alpha_{i}$ true, which implies $(\mathcal{M}, s) \equiv_{r}\left(\mathcal{M}, s^{\prime}\right)$. This proves the claim.

Given $C \subseteq \Sigma^{*}$ and $s \in \Sigma^{*}$, let $d(s, C)$ be $|s|-|\operatorname{Meet}(s, C)|$, that is, the length of the relative suffix of Meet $(s, C)$ in $s$.

Given a database $D$, let $\operatorname{prefix}(D)=\left\{s \mid s \preceq s^{\prime}, s^{\prime} \in \operatorname{adom}(D)\right\}$.

Lemma 4.20 Let $\varphi(x)$ be a $\mathrm{RC}(\mathbf{S})$ query. Then there exists a number $k>0$, such that the following holds. If $D \models \varphi(s)$ for some $s$ with $d(s$, prefix $(D))>k$ then there are infinitely many strings $c$ such that $D \models \varphi(c)$. If $\varphi$ only uses prefix-restricted quantification then $k$ can be effectively computed.

Proof of Lemma 4.20. By Corollary 4.4 we may assume without loss of generality that all quantification in $\varphi$ is prefixrestricted. Let $r$ be the quantifier rank of $\varphi$. We show that we can find $k$ such that the following holds. Let $D$ be a database, and $s$ a string with $d(s, \operatorname{prefix}(D))>k$. For a string $u$, let $C_{u}=\operatorname{prefix}(D) \cup\left\{s^{\prime} \mid s^{\prime} \preceq u\right\}$. Then there are infinitely many strings $u$ such that the duplicator has a winning strategy for the $r$-round Ehrenfeucht game on $C_{s}$ and $C_{u}$ (with the partial isomorphism being with respect to the operations of $\mathbf{S}$, and with $s$ mapped to $u$ ); moreover, in the winning strategy, the duplicator simply copies the spoiler's moves on prefix $(D)$. Note that this condition implies that in the final position all the $S C$-relations are preserved, and hence $D \models \varphi(s)$ iff $D \models \varphi(u)$, thus implying the lemma.

To prove the above condition, let $k>0$ be given by the claim. Consider $s$ with $d(s$, prefix $(D))>k$, and let $s^{\prime}$ be the relative suffix of $\operatorname{Meet}(s, \operatorname{prefix}(D))$ in $s$. We have $\left|s^{\prime}\right|>k$. We then have infinitely many strings $u^{\prime}$ such that $\left(\mathbf{S}, s^{\prime}\right) \equiv_{r}\left(\mathbf{S}, u^{\prime}\right)$. Take any such string $u^{\prime}$, and form a new string $u=(\operatorname{Meet}(s, \operatorname{prefix}(D))) \cdot u^{\prime}$. It is clear that the required strategy exists for the duplicator on $C_{s}$ and $C_{u}$.

To show that $k$ can be found from $\varphi$, note first that the conversion into a query with prefix-bounded quantification is effective, and the claim is effective too, as any $\mathbf{S}_{\text {len }}$ formula can be effectively converted into an automaton. The lemma is proved.

Next we define $\downarrow D=\left\{s|| s|\leq| s^{\prime} \mid, s^{\prime} \in \operatorname{adom}(D)\right\}$. 
Lemma 4.21 Let $\varphi(x)$ be a $\mathrm{RC}\left(\mathbf{S}_{\text {len }}\right)$ query. Then there exists a number $k>0$ such that the following holds. If $D \models \varphi(s)$ for some $s$ with $d(s, \downarrow D)>k$ then there are infinitely many strings $c$ such that $D \models \varphi(c)$. If $\varphi$ only uses length-restricted quantification then $k$ can be effectively computed.

Proof of Lemma 4.21. By Proposition 4.8 we may assume without loss of generality that in $\varphi(x)$ all quantification is length-restricted. Let $r$ be the quantifier rank of $\varphi$. For any string $s$, let $\mathbf{S}_{\text {len }}^{s}$ be the structure $\left(\downarrow s, \prec,\left(L_{a}\right)_{a \in \Sigma}\right.$, el, $\left.s\right)$. By the Claim, we can find a number $k$ such that for any string $s$ of $|s|>k$, there exist infinitely many strings $s^{\prime}$ of $\left|s^{\prime}\right|>k$ with $\mathbf{S}_{\text {len }}^{s} \equiv_{r} \mathbf{S}_{\text {len }}^{s^{\prime}}$. Note that $k$ can be found effectively for a given $\varphi$.

Now assume that for some $D$ and $s, D \models \varphi(s)$ with $d(s, \downarrow D)>k$. Let $m$ be the maximum length of a string in $\operatorname{adom}(D)$, and $s_{0}$ the prefix of $s$ of length $m$. Then $s=s_{0} \cdot s_{1}$ for a string $s_{1}$ of $\left|s_{1}\right|>k$. We now show that there are infinitely many strings $s^{\prime}$ of length greater than $m+k$ such that the duplicator has a winning strategy in the $r$-round Ehrenfeucht game on $\mathbf{S}_{\text {len }}^{s}$ and $\mathbf{S}_{\text {len }}^{s^{\prime}}$ such that the play is the identity function when restricted to strings of length not exceeding $m$. Clearly, this suffices to prove the lemma, since $|x| \leq m$ for all $x \in \operatorname{adom}(D)$ and thus $(D, s) \equiv_{r}\left(D, s^{\prime}\right)$ and $D \models \varphi\left(s^{\prime}\right)$.

Consider any string $s_{1}^{\prime}$ such that $\mathbf{S}_{\text {len }}^{s_{1}} \equiv_{r} \mathbf{S}_{\text {len }}^{s_{1}^{\prime}}$ (we know that there are infinitely many of them), and let $s^{\prime}$ be $s_{0} \cdot s_{1}^{\prime}$. We prove that the duplicator wins the $r$-round game on $\mathbf{S}_{\text {len }}^{s}$ and $\mathbf{S}_{\text {len }}^{s^{\prime}}$. The strategy is as follows. The duplicator maintains (for his memory) a separate game on $\mathbf{S}_{\text {len }}^{s_{1}}$ and $\mathbf{S}_{\text {len }}^{s_{1}^{\prime}}$. If the spoiler plays a string of length not exceeding $m$, the duplicator's response is the same string. Assume that the spoiler plays $x$ of $|x|>m$. Let $x=x_{0} \cdot x_{1}$ with $x_{0}$ being the length $m$ prefix of $x$. Assume that the spoiler plays it in $\mathbf{S}_{\text {len }}^{s}$ (if the spoiler plays in $\mathbf{S}_{\text {len }}^{s^{\prime}}$, the proof is identical). The duplicator then looks at the current position of the auxiliary game on $\mathbf{S}_{\text {len }}^{s_{1}}$ and $\mathbf{S}_{\text {len }}^{s_{1}^{\prime}}$ (which is empty until the spoiler makes the first move of length $>m$ ), and extends it by one move: spoiler's move is $x_{1}$ on $\mathbf{S}_{\text {len }}^{s_{1}}$, and the response is a string $x_{1}^{\prime}$ in $\mathbf{S}_{\text {len }}^{s_{1}^{\prime}}$ according to the winning strategy $\mathbf{S}_{\text {len }}^{s_{1}} \equiv_{r} \mathbf{S}_{\text {len }}^{s_{1}^{\prime}}$. Having done that, the duplicator returns to the game on $\mathbf{S}_{\text {len }}^{s}$ and $\mathbf{S}_{\text {len }}^{s^{\prime}}$, and responds by $x_{0} \cdot x_{1}^{\prime}$ in $\mathbf{S}_{\text {len }}^{s^{\prime}}$.

We now show that the duplicator wins the game. Clearly all $L_{a}$ predicates are preserved. Assume that in $\mathbf{S}_{\text {len }}^{s}$, $u \prec v$, where $u$ and $v$ are two moves in the game. Let $u^{\prime}$ and $v^{\prime}$ be the corresponding moves played on $\mathbf{S}_{\text {len }}^{s^{\prime}}$. If both $u$ and $v$ are of length at most $m$, then $u^{\prime}=u, v^{\prime}=v$ and $u^{\prime} \prec v^{\prime}$. If $|u| \leq m$ and $|v|>m$, then $u^{\prime}=u$, and $v^{\prime}$ is of the form $v_{0} \cdot v_{1}^{\prime}$, where $v_{0}$ is the prefix of $v$ of length $m$, and thus $u^{\prime} \prec v^{\prime}$. If $|u|,|v|>m$ then $u^{\prime} \prec v^{\prime}$ by the winning strategy on $\mathbf{S}_{\text {len }}^{s}$ and $\mathbf{S}_{\text {len }}^{s^{\prime}}$ and the fact that $u$ and $v$ have the same prefix of length $m$. Next, assume el $(u, v)$ holds. The case of the length $\leq m$ is trivial. If $|u|,|v|>m$, then $u=u_{0} \cdot u_{1}, v=v_{0} \cdot v_{1}$, where $u_{0}, v_{0}$ are length $m$ prefixes, and by the description of the duplicator's strategy, $u^{\prime}=u_{0} \cdot u_{1}^{\prime}$ and $v^{\prime}=v_{0} \cdot v_{1}^{\prime}$, where $u_{1}^{\prime}$, $v_{1}^{\prime}$ are moves taken from the auxiliary game on $\mathbf{S}_{\text {len }}^{s_{1}}$ and $\mathbf{S}_{\text {len }}^{s_{1}^{\prime}}$. Since the duplicator wins the auxiliary game, we have $\left|u_{1}\right|=\left|u_{1}^{\prime}\right|$ and $\left|v_{1}\right|=\left|v_{1}^{\prime}\right|$, and thus el $\left(u^{\prime}, v^{\prime}\right)$ holds. This completes the proof of the lemma.

For any set $X$, let $N_{p}^{0}(X)=\left\{s-s_{1}+s_{2}|s \in X,| s_{1}|,| s_{2} \mid \leq p\right\}$, and let $N_{p}(X)=\operatorname{prefix}\left(N_{p}^{0}(X)\right)$ (that is, the prefix-closure of $\left.N_{p}^{0}(X)\right)$. Note that $N_{p}(X)=N_{p}^{0}(\operatorname{prefix}(X))$, and $N_{k}\left(N_{m}(X)\right) \subseteq N_{k+m}(X)$.

Lemma 4.22 Let $\varphi(x)$ be a $\mathrm{RC}\left(\mathbf{S}_{\text {left }}\right)$ query. Then there exist numbers $l, m>0$ such that the following holds. If $D \models \varphi(s)$ for some $s$ with $d\left(s, N_{m}(\right.$ prefix $\left.(D))\right)>l$ then there are infinitely many strings $c$ such that $D \models \varphi(c)$.

Proof of Lemma 4.22. This follows from the normal form for $\mathbf{S}_{\text {left }}$ (Corollary 3.15) and Lemma 4.20.

Lemma 4.23 Given a $\mathrm{RC}\left(\mathbf{S}_{\mathrm{reg}}\right)$ query $\varphi(x)$, there exists $k>0$ such that whenever $D \models \varphi(s)$ with $d(s, \operatorname{prefix}(D))>$ $k$, there are infinitely many strings $c$ such that $D \models \varphi(c)$.

Proof of Lemma 4.23. To show this, assume by the restricted quantifier collapse and quantifier-elimination for $\mathbf{S}_{\text {reg }}^{+}$ that $\varphi$ is of the form

$$
Q y_{1} \in \operatorname{adom} \ldots Q y_{l} \in \operatorname{adom} \bigvee_{i} \bigwedge_{j} \alpha_{i j}(x, \vec{y})
$$

where each $\alpha_{i j}$ is either an atomic or negated atomic $S C$-formula, or an $\mathbf{S}_{\text {reg }}$ formula not involving the variable $x$, or a formula of the form $P_{L}\left(t_{1}(x, \vec{y}), t_{2}(x, \vec{y})\right)$, where $t_{i}$ is either $\epsilon$ or a $\sqcap$-term. Let $L_{1}, \ldots, L_{m}$ be the regular languages such that the formulae $P_{L_{i}}$ appear in $\varphi$. We denote the quantifier-free part (that is $\bigvee_{i} \bigwedge_{j} \alpha_{i j}$ ) by $\beta(x, \vec{y})$. 
Let $i>1$ and $D \models \varphi(s)$ with $d(s, \operatorname{prefix}(D))>k$. We apply Lemma 4.19 to $z=s-(\operatorname{Meet}(s, \operatorname{prefix}(D)))$, and let $c=(\operatorname{Meet}(s, \operatorname{prefix}(D))) \cdot u v^{i} w, i>1$. We now show that for every $\vec{y}_{0} \in(\operatorname{adom}(D) \cup\{\epsilon\})^{l}$, it is the case that $D \models \beta\left(s, \vec{y}_{0}\right)$ iff $D \models \beta\left(c, \vec{y}_{0}\right)$. This will imply $D \models \varphi(s) \leftrightarrow \varphi(c)$ (see [10]) thus proving the result. To prove $D \models \beta\left(s, \vec{y}_{0}\right) \leftrightarrow \beta\left(c, \vec{y}_{0}\right)$, it suffices to show that $D \models P_{L}\left(t_{1}\left(c, \vec{y}_{0}\right), t_{2}\left(c, \vec{y}_{0}\right)\right) \leftrightarrow P_{L}\left(t_{1}\left(s, \vec{y}_{0}\right), t_{2}\left(s, \vec{y}_{0}\right)\right)$, where $L \in\left\{L_{1}, \ldots, L_{m}\right\}$, as for all other types of formulae $\alpha_{i j}$ the equivalence is trivial.

We now fix $\vec{y}_{0} \in(\operatorname{adom}(D) \cup\{\epsilon\})^{l}$ and consider the atomic formula $\chi(x)=P_{L}\left(t_{1}\left(x, \vec{y}_{0}\right), t_{2}\left(x, \vec{y}_{0}\right)\right)$. If $t_{j}$, $j=1,2$ involves meets of $x$ with some of the components of $\vec{y}_{0}$, then the value of $t_{j}$ will be the same on $s$ and on $c$, as $\operatorname{Meet}(s, \operatorname{prefix}(D))=\operatorname{Meet}(c, \operatorname{prefix}(D))$. Thus, if both $t_{1}$ and $t_{2}$ involve such meets, we have $D \models \chi(s) \leftrightarrow \chi(c)$.

The other case is when $t_{2}$ is simply $x$, and in this case $t_{1}$ is either $\epsilon$ or $x \sqcap y_{0}^{i_{1}} \sqcap \ldots \sqcap y_{0}^{i_{p}}$, for some components of $\vec{y}_{0}$ (we can include $x$ in the $\sqcap$-term without loss of generality, since its value must be a prefix of $x$, by the definition of $\left.P_{L}\right)$. Since $\operatorname{Meet}(s, \operatorname{prefix}(D))=\operatorname{Meet}(c, \operatorname{prefix}(D)), t_{1}(s)$ equals $t_{1}(c)$ and belongs to prefix $(D)$. To prove $D \models \chi(s) \leftrightarrow \chi(c)$, it then suffices to show that $s-s_{0} \in L$ iff $c-s_{0} \in L$, which follows immediately from Lemma 4.19. This completes the proof of the lemma.

Finally, we need a lemma for $\mathbf{S}_{\text {reg,left }}$. Its proof follows from the normal form for $\mathbf{S}_{\text {reg,left }}$ (Corollary 3.25 ) and Lemma 4.23.

Lemma 4.24 Let $\varphi(x)$ be a $\mathrm{RC}\left(\mathbf{S}_{\mathrm{reg}, \mathrm{left}}\right)$ query. Then there exist numbers $l, m>0$ such that the following holds. Assume that $D \models \varphi(s)$ for some $s$ with $d\left(s, N_{m}(\operatorname{prefix}(D))\right)>l$. Then there are infinitely many strings $c$ such that $D \models \varphi(c)$.

Proof of Theorem 4.18, completed. To prove the theorem, take an arbitrary query $\psi(\vec{y})$ and form $\varphi(x)$ that defines the active domain of the output of $\psi$, that is, $\varphi(x)$ is

$$
\exists y_{2}, \ldots, y_{n} \psi\left(x, y_{2}, \ldots, y_{n}\right) \vee \ldots \vee \exists y_{1}, \ldots, y_{n-1} \psi\left(y_{1}, \ldots, y_{n-1}, x\right)
$$

It then suffices to prove the theorem for $\varphi(x)$, since $\psi$ is safe for $D$ iff $\varphi$ is safe for $D$, and thus for any $\gamma$ such that $(\gamma, \varphi)$ is equivalent to $\varphi$ on all $D$ for which $\varphi$ is safe, the same would be true for $(\gamma, \psi)$ and $\psi$.

Having reduced the problem to queries on one variable, simply apply the corresponding lemmas. For $\mathrm{RC}(\mathbf{S})$, given $\varphi(x)$, find the number $k$ as in Lemma 4.20, and let $\gamma(x, y)$ say that $x$ is a prefix of the string of the form $y \cdot s$ with $|s| \leq k$. From Lemma 4.20 it follows that $(\gamma, \varphi)$ is equivalent to $\varphi$ on any $D$ for which $\varphi$ is safe. Finally, $\gamma$ is clearly algebraic, and expressible over $\mathbf{S}$ for any fixed $k$.

For $\operatorname{RC}\left(\mathbf{S}_{\text {len }}\right)$, given $\varphi(x)$, we get $k$ from Lemma 4.21 and let $\gamma(x, y)$ be an $\mathbf{S}_{\text {len }}$ formula saying that the length of $x$ is at most the length of $y$ plus $k$. Clearly, this is expressible for each fixed $k$, and $(\gamma, \varphi)$ coincides with $\varphi$ on any $D$ for which $\varphi$ is safe. This completes the proof of the theorem.

The proof for $\mathbf{S}_{\text {left }}$ is similar: one gets $l, t$ from Lemma 4.22, and the formula $\gamma(x, y)$ says that $x$ is at the distance at most $l$ from a prefix of a string of the form $y-e+f$, with $|e|,|f| \leq t$. The proofs for $\mathbf{S}_{\text {reg }}$ and $\mathbf{S}_{\text {reg,left }}$ follow the same idea. This concludes the proof of Theorem 4.18 .

Corollary 4.25 For each of

- $\mathrm{RC}(\mathbf{S})$,

- $\mathrm{RC}\left(\mathbf{S}_{\text {left }}\right)$,

- $\mathrm{RC}\left(\mathbf{S}_{\mathrm{reg}}\right)$,

- $\mathrm{RC}\left(\mathbf{S}_{\text {reg,left }}\right)$,

- $\mathrm{RC}\left(\mathbf{S}_{\mathrm{len}}\right)$,

the classes of range-restricted and safe queries coincide, and safe queries have effective syntax.

Note that for queries in $\mathrm{RC}(\mathbf{S})$ and $\mathrm{RC}\left(\mathbf{S}_{\text {len }}\right)$ that use a restricted form of quantification (prefix or length), the proof gives us a stronger result: namely, the formula $\gamma$ can be effectively found for a given $\varphi$. 


\subsubsection{Relational algebras}

It is a classical result of relational database theory that the set of safe relational calculus queries is precisely the set of relational algebra queries [1]. This result extends to string calculi considered here: safety theorems proved earlier can be used to show that safe queries in $\operatorname{RC}(\mathbf{S})$ and $\operatorname{RC}\left(\mathbf{S}_{\text {len }}\right)$ can be captured by appropriate extensions of relational algebra.

Let safe_RC $(\mathcal{M})$ be the class of all safe queries in $\operatorname{RC}(\mathcal{M})$. To define algebras capturing safe_RC $(\mathcal{M})$ for the previous two structures, we need a number of operations extending the usual relational algebra (that is, selection $\sigma$, projection $\pi$, cartesian product $\times$, difference - , union $\cup$ ):

$R_{\epsilon}$ : is the constant unary relation $\{\epsilon\}$.

$\sigma_{\alpha}$ : for a formula $\alpha\left(x_{1}, \ldots, x_{n}\right)$. On an $n$-attribute relation $R$, it returns the set of tuples $\left(s_{1}, \ldots, s_{n}\right)$ from $R$ such that $\alpha\left(s_{1}, \ldots, s_{n}\right)$ holds.

$\operatorname{prefix}_{i}:$ On an $m$-attribute relation $R$, it returns the $m+1$-attribute relation $\left\{\left(s_{1}, \ldots, s_{m+1}\right) \mid\left(s_{1}, \ldots, s_{m}\right) \in R, s_{m+1} \preceq\right.$ $\left.s_{i}\right\}$.

$\operatorname{addl}_{i}^{a}, a \in \Sigma$ : On an $m$-attribute relation $R$, it returns the $m+1$-attribute relation $\left\{\left(s_{1}, \ldots, s_{m+1}\right) \mid\left(s_{1}, \ldots, s_{m}\right) \in R, s_{m+1}=\right.$ $\left.s_{i} \cdot a\right\}$.

$\downarrow_{i}$ : Given an $m$-attribute relation $R, \downarrow_{i}(R)$ returns $\left\{\left(s_{1}, \ldots, s_{m+1}\right)\left|\left(s_{1}, \ldots, s_{m}\right) \in R,\right| s_{m+1}|\leq| s_{i} \mid\right\}$.

$\operatorname{addf}_{i}^{a}, a \in \Sigma:$ On an $m$-attribute relation $R$, it returns the $m+1$-attribute relation $\left\{\left(s_{1}, \ldots, s_{m+1}\right) \mid\left(s_{1}, \ldots, s_{m}\right) \in\right.$ $\left.R, s_{m+1}=a \cdot s_{i}\right\}$.

$\operatorname{trim}_{i}^{a}, a \in \Sigma:$ On an $m$-attribute relation $R$, it returns the $m+1$-attribute relation $\left\{\left(s_{1}, \ldots, s_{m+1}\right) \mid\left(s_{1}, \ldots, s_{m}\right) \in\right.$ $\left.R, s_{m+1}=s_{i}-a\right\}$.

It should be pointed out that the formula $\alpha$ in $\sigma_{\alpha}$ does not refer to the database.

We now define the relational algebras:

$\mathrm{RA}(\mathbf{S})$ extends relational algebra with $R_{\epsilon}, \sigma_{\alpha}$, where $\alpha$ ranges over $\mathrm{FO}(\mathbf{S})$ formulae, $\operatorname{prefix}_{i}$ and $\operatorname{addl}_{i}^{a}$.

$\mathrm{RA}\left(\mathbf{S}_{\text {len }}\right)$ extends relational algebra with $R_{\epsilon}, \sigma_{\alpha}$, where $\alpha$ ranges over $\mathrm{FO}\left(\mathbf{S}_{\text {len }}\right)$ formulae, $\downarrow_{i}$, prefix ${ }_{i}$, and addl ${ }_{i}^{a}$.

$\operatorname{RA}\left(\mathbf{S}_{\text {left }}\right)$ is the extension of relational algebra with $\sigma_{\alpha}$ (where $\alpha$ ranges over $\mathbf{S}_{\text {left }}$ formulae), prefix, addf ${ }_{i}^{a}$ and $\operatorname{trim} i_{i}^{a}$.

$\mathrm{RA}\left(\mathbf{S}_{\text {reg }}\right)$ extends relational algebra with $R_{\epsilon}, \sigma_{\alpha}$, where $\alpha$ ranges over $\mathrm{FO}\left(\mathbf{S}_{\text {reg }}\right)$ formulae, $\operatorname{prefix}_{i}$ and addl $i_{i}^{a}$.

$\mathrm{RA}\left(\mathbf{S}_{\text {reg,left }}\right)$ extends relational algebra with $R_{\epsilon}, \sigma_{\alpha}$, where $\alpha$ ranges over $\mathrm{FO}\left(\mathbf{S}_{\text {reg,left }}\right)$ formulae, $\operatorname{prefix}_{i}$, addl ${ }_{i}^{a}$ and $\operatorname{trim}_{i}^{a}$.

Theorem 4.26 • safe_RC $(\mathbf{S})=\mathrm{RA}(\mathbf{S})$;

- safe_RC $\left(\mathbf{S}_{\text {len }}\right)=\operatorname{RA}\left(\mathbf{S}_{\text {len }}\right)$;

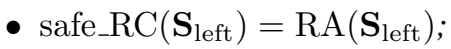

- safe_RC $\left(\mathbf{S}_{\text {reg }}\right)=\operatorname{RA}\left(\mathbf{S}_{\text {reg }}\right)$;

- safe_RC $\left(\mathbf{S}_{\text {reg, left }}\right)=\operatorname{RA}\left(\mathbf{S}_{\text {reg,left }}\right)$.

Proof. We start with $\mathrm{RA}(\mathbf{S})$. Every $\mathrm{RA}(\mathbf{S})$ expression produces a finite result, and the standard translation from algebra to calculus (extended with rules for addl and prefix) shows $\mathrm{RA}(\mathbf{S}) \subseteq \mathrm{RC}(\mathbf{S})$.

For the converse, let $\varphi(\vec{x})$ be a safe $\operatorname{RC}(\mathbf{S})$ query. By Theorem 4.18, on every database $D$, the active domain of the output of $\varphi$ on $D$ is contained in the set $V_{k}[D]=\{x \mid d(x, \operatorname{prefix}(D)) \leq k\}$ for some $k \geq 0$.

We first note that $V_{k}[D]$ is definable by an $\operatorname{RA}(\mathbf{S})$ expression. Indeed, the active domain of $D$ is definable in relational algebra. Next, for each fixed string $s$ and a finite set $S$, there is an expression addl ${ }^{s}$ that defines the 
set $\left\{\left(s^{\prime}, s^{\prime} \cdot s\right) \mid s^{\prime} \in S\right\}$ simply by composing addl ${ }^{a}$ operations. Thus, for $S=$ adom $(D)$, we define $S^{\prime}=$ $\bigcup_{|s| \leq k} \operatorname{addl}^{s}(S)$, and note that $V_{k}[D]=\pi_{3}\left(\operatorname{prefix}_{2}\left(S^{\prime}\right)\right)$.

Let $D_{V_{k}[D]}$ be the extension of $D$ by one unary predicate interpreted as $V_{k}[D]$. Since $\varphi$ is safe, every element of every tuple in $\varphi(D)$ belongs to $V_{k}[D]$. We know that in order to evaluate $\varphi(\vec{x})$, it suffices to restrict quantification to the prefix-closure of $\operatorname{adom}(D)$ and $\vec{x}$. Since $V_{k}[D]$ is prefix-closed, this implies that there is an active-domain query $\varphi^{\prime}(\vec{x})$ over the schema extended with one unary symbol such that $\varphi^{\prime}\left(D_{V_{k}[D]}\right)=\varphi(D)$ (here active-domain means that all quantification is restricted to the active domain, and that the output is only considered within the active domain of the input). By [10], $\varphi^{\prime}$ can be expressed by relational algebra extended with $\sigma_{\alpha}$, for $\alpha$ ranging over $\mathbf{S}$ formulae. Since $D_{V_{k}[D]}$ is expressible in $\operatorname{RA}(\mathbf{S})$ and $\varphi(D)=\varphi^{\prime}\left(D_{V_{k}[D]}\right)$, we conclude that $\varphi$ is expressible in $\operatorname{RA}(\mathbf{S})$.

The proof for $\mathbf{S}_{\text {len }}$ is almost identical, except that one defines $V_{k}[D]$ as $\{x|| x|\leq| y \mid+k, y \in \operatorname{adom}(D)\}$, which is expressible in $\operatorname{RA}\left(\mathbf{S}_{\text {len }}\right)$ using the $\operatorname{addl}^{s}$ operations and the operations $\downarrow_{i}$.

The proof for $\mathbf{S}_{\mathrm{reg}}$ is identical to the proof of for $\mathbf{S}$, as the set $V_{k}[D]$ is expressible in $\mathrm{RA}\left(\mathbf{S}_{\mathrm{reg}}\right)$. For $\mathbf{S}_{\text {left }}$, the proof again follows the same lines: all that is needed is that the set $N_{p}(\operatorname{adom}(D))$ is expressible in $\operatorname{RA}\left(\mathbf{S}_{\text {left }}\right)$ for a fixed $p$. But this follows from the fact that $\operatorname{adom}(D)$ is definable in relational algebra, using prefix, addf ${ }_{i}^{a}$ and $\operatorname{trim} i_{i}^{a}$ it is then possible to define $N_{p}(\operatorname{adom}(D))$. The proof for $\mathbf{S}_{\mathrm{reg}, \text { left }}$ follows from the expressibility of $V_{k}[D]$ and $N_{p}(\operatorname{adom}(D))$.

One of the operations in $\operatorname{RA}\left(\mathbf{S}_{\text {len }}\right), \downarrow_{i}$, is very expensive, as it may create sets whose size is exponential in the size of the input. This seems, however, unavoidable, as there are very expensive (e.g., NP-complete) safe queries in $\mathrm{RC}\left(\mathbf{S}_{\text {len }}\right)$.

\subsubsection{Deciding Safety Properties of Queries}

Although query safety is undecidable for pure relational calculus (and hence for any extension), state-safety (given a query $\varphi$ and a database $D$, is $\varphi(D)$ finite?) is decidable [64]. State safety is also known to be decidable for various extensions of the form $\mathrm{RC}(\mathcal{M})$ (for example, for the natural numbers with successor [64] or the real field [11]). For $\mathrm{RC}(\mathbf{S})$ and $\mathrm{RC}\left(\mathbf{S}_{\text {len }}\right)$, this decidability holds as well:

Proposition 4.27 State-safety is decidable for $\mathrm{RC}(\mathcal{M})$, where $\mathcal{M}$ is one of $\mathbf{S}, \mathbf{S}_{\text {left }}, \mathbf{S}_{\mathrm{reg}}$, $\mathbf{S}_{\text {reg,left }}, \mathbf{S}_{\text {len }}$.

Proof. Given a query $\varphi(\vec{x})$ and a database $D$, we obtain a formula $\varphi^{\prime}(\vec{x})$ by replacing each occurrence of a schema predicate $S(\vec{z})$ by a disjunction $\vec{z}=\vec{t}_{1} \vee \ldots \vee \vec{z}=\vec{t}_{m}$ where $\left\{t_{1}, \ldots, t_{m}\right\}$ is the interpretation of $S$ in $D$. Since the formula $z=s$ is definable in all the structures for every fixed $s, \varphi^{\prime}$ can thus be viewed as a formula over $\mathbf{S}_{\text {len }}$ such that $\mathbf{S}_{\text {len }} \models \varphi^{\prime}(\vec{x})$ iff $D \models \varphi(\vec{x})$. We now consider the sentence $\Phi$ defined as

$$
\exists \vec{y} \forall \vec{x}\left(\varphi^{\prime}(\vec{x}) \rightarrow \exists \vec{z}\left(\bigwedge_{i} z_{i} \prec y_{i} \wedge \operatorname{el}\left(z_{i}, x_{i}\right)\right)\right)
$$

Then $\varphi(D)$ is finite iff $\left\{\vec{a} \mid \mathbf{S}_{\text {len }} \models \varphi^{\prime}(\vec{a})\right\}$ is finite iff $\mathbf{S}_{\text {len }} \models \Phi$, and thus the state-safety is decidable, since the theory of $\mathbf{S}_{\text {len }}$ is decidable.

As query safety is undecidable, one often considers restrictions for which decidability can be obtained. Here we look at one of the most fundamental classes of queries - conjunctive queries. We take their definition in the context of interpreted operations from $[11,46]$. A conjunctive query in $\mathrm{RC}(\mathcal{M})$ is a query of the form

$$
\varphi(\vec{x}) \equiv \exists \vec{y} \bigwedge_{i=1}^{k} S_{i}\left(\vec{u}_{i}\right) \wedge \gamma(\vec{x}, \vec{y}),
$$

where $k \geq 0$, each $S_{i}$ is a schema relation, $\vec{u}_{i}$ is a subtuple of $(\vec{x}, \vec{y})$ of the same arity as $S_{i}$, and $\gamma$ is an $\mathcal{M}$ formula. A Datalog-like notation for such a query would be $\varphi(\vec{x}):-S_{1}\left(\vec{u}_{1}\right), \ldots, S_{k}\left(\vec{u}_{k}\right), \gamma(\vec{x}, \vec{y})$.

In [11], safety of conjunctive queries was shown decidable for $\operatorname{RC}(\mathcal{M})$, for various structures $\mathcal{M}$ on the reals with numerical operations. We now show a general result from which the decidability results for string structures $\mathbf{S}, \mathbf{S}_{\text {len }}$ as well as those considered in [11] follow. We say that finiteness is definable with parameters in $\mathcal{M}$ if for each formula $\psi(\vec{x}, \vec{y})$ in $\mathcal{M}$, there exists another formula $\psi_{\text {fin }}(\vec{y})$ such that $\mathcal{M} \models \psi_{\text {fin }}(\vec{a})$ iff the set $\{\vec{b} \mid \mathcal{M} \models \psi(\vec{b}, \vec{a})\}$ is finite. Furthermore, $\psi_{\text {fin }}(\vec{y})$ can be computed effectively. 
Theorem 4.28 Assume that $\mathcal{M}$ can be expanded to $\mathcal{M}^{\prime}$ such that the theory of $\mathcal{M}^{\prime}$ is decidable, and finiteness is definable with parameters in $\mathcal{M}^{\prime}$. Then safety of Boolean combinations of conjunctive queries in $\mathrm{RC}(\mathcal{M})$ is decidable.

Proof. We start with a few easy observations about Boolean combinations of conjunctive queries in $\mathrm{RC}(\mathcal{M})$. First, if $\alpha(\vec{x})$ is a conjunctive query, it can be represented in the form $\exists \vec{z} \in \operatorname{adom} \bigwedge_{i} S_{i}\left(\vec{u}_{i}\right) \wedge \gamma(\vec{x}, \vec{z})$. Indeed, given a query $\exists \vec{y} \bigwedge_{i} S_{i}\left(\vec{u}_{i}\right) \wedge \gamma^{\prime}(\vec{x}, \vec{y})$, let $\vec{z}$ be the subtuple of $\vec{y}$ that consists of $y_{j}$ s appearing in the $S_{i}$ atoms. Then the query can be rewritten to the one with active-domain quantification only, where $\gamma(\vec{x}, \vec{z}) \equiv \exists \vec{v} \gamma^{\prime}(\vec{x}, \vec{y})$ - here $\vec{v}$ lists those variables in $\vec{y}$ that do not belong to $\vec{z}$. We also note that every conjunctive query is monotone.

Next, every Boolean combination of conjunctive queries is equivalent to a union of queries of the form $\alpha(\vec{x}) \wedge$ $\neg \beta_{1}(\vec{x}) \wedge \ldots \wedge \neg \beta_{k}(\vec{x})$, where $k>0$, and $\alpha, \beta_{1}, \ldots, \beta_{k}$ are conjunctive queries. Indeed, one puts a given Boolean combination in DNF, and observes that a conjunction of two conjunctive queries is a conjunctive query again, and since true and false are by definition conjunctive queries, we can assume that $k>0$ and that one conjunctive query is present without negation.

Thus, we must show that it is decidable whether a query $q(\vec{x})$ of the form $\alpha(\vec{x}) \wedge \neg \beta_{1}(\vec{x}) \wedge \ldots \wedge \neg \beta_{k}(\vec{x})$ is safe. Let $\alpha(\vec{x})$ be $\exists \vec{z} \in$ adom $\bigwedge_{i=1}^{l} S_{i}\left(\vec{u}_{i}\right) \wedge \gamma(\vec{x}, \vec{z})$.

We show the following claim: if there exists a database $D$ such that $q(D)$ is infinite, then there exists a database $D^{\prime}$ with at most $l$ tuples such $q\left(D^{\prime}\right)$ is finite. This in turn follows from the following: let $\mathcal{D}_{l}$ be the set of all databases $D^{\prime}$ with at most $l$ tuples such that $D^{\prime} \subseteq D$. Then $\alpha(D)=\cup_{D^{\prime} \in \mathcal{D}_{l}} \alpha\left(D^{\prime}\right)$. Indeed, the $\supseteq$ inclusion follows from monotonicity, and the $\subseteq$ inclusion from the fact that to witness $\vec{a} \in \alpha(D)$, it suffices to find $\overrightarrow{\vec{b}}$ such that $\bigwedge_{i=1}^{l} S_{i}\left(\vec{u}_{i}\right) \wedge$ $\gamma(\vec{a}, \vec{b})$ holds; if such $\vec{b}$ exists, the $l$ tuples $S_{i}\left(\vec{u}_{i}\right)$ form a database $D^{\prime}$ for which $\vec{a} \in \alpha\left(D^{\prime}\right)$.

Now, suppose $q(D)$ is infinite, and $D$ has more than $l$ tuples. We have $\alpha(D)=\bigcup_{D^{\prime} \in \mathcal{D}_{l}} \alpha\left(D^{\prime}\right)$, and thus $q(D)=$ $\bigcup_{D^{\prime} \in \mathcal{D}_{l}}\left(\alpha\left(D^{\prime}\right) \cap \bigcap_{i} \neg \beta_{i}(D)\right) \subseteq \bigcup_{D^{\prime} \in \mathcal{D}_{l}}\left(\alpha\left(D^{\prime}\right) \cap \bigcap_{i} \neg \beta_{i}\left(D^{\prime}\right)\right)$, since $\neg \beta_{i}$ s are antimonotone. Since $q(D)$ is infinite, for some $D^{\prime} \in \mathcal{D}_{l}, q\left(D^{\prime}\right)=\alpha\left(D^{\prime}\right) \cap \bigcap_{i} \neg \beta_{i}\left(D^{\prime}\right)$ is infinite. This proves the claim.

Let $\overrightarrow{\mathbf{t}}$ stand for $\vec{t}_{1}^{1}, \ldots, \vec{t}_{l}^{1}, \ldots, \vec{t}_{1}^{p}, \ldots, \vec{t}_{l}^{p}$, where $p$ is the number of relation symbols in $S C$, and $\vec{t}_{j}^{t}$ is a tuple of variables of the same length as the arity of $S_{i}$. For a query $q$ of the form $\alpha(\vec{x}) \wedge \neg \beta_{1}(\vec{x}) \wedge \ldots \wedge \neg \beta_{k}(\vec{x})$, let $q^{\prime}(\vec{x}, \overrightarrow{\mathbf{t}})$ be the $\mathcal{M}$ formula obtained by replacing each $S_{i}(\vec{u})$ with $\bigvee_{j=1}^{l} \vec{u}=\vec{t}_{j}$. Then $\mathcal{M} \models q^{\prime}(\vec{x}, \overrightarrow{\mathbf{t}})$ iff $D_{\overrightarrow{\mathbf{t}}}=q(\vec{x})$, where $D_{\overrightarrow{\mathbf{t}}}$ is the database in which $S_{i}$ is interpreted as $\left\{\vec{t}_{1}^{t}, \ldots, \vec{t}_{l}^{2}\right\}$. By the assumptions on $\mathcal{M}$, we know that in the expanded model we have a formula $q_{\text {fin }}^{\prime}(\overrightarrow{\mathbf{t}})$ such that $\mathcal{M}^{\prime} \models q_{\text {fin }}^{\prime}(\overrightarrow{\mathbf{t}})$ iff the set of $\vec{x}$ such that $q^{\prime}(\vec{x}, \overrightarrow{\mathbf{t}})$ holds is finite. In other words, it holds iff $q\left(D_{\overrightarrow{\mathbf{t}}}\right)$ is finite. Hence, the sentence $\forall \overrightarrow{\mathbf{t}} q_{\text {fin }}^{\prime}(\overrightarrow{\mathbf{t}})$ is true in $\mathcal{M}$ iff $q(D)$ is finite for every database with at most $l$ tuples, which by the previous claim means that $q$ is safe. The decidability of the theory of $\mathcal{M}^{\prime}$ now implies the decidability of the safety of $q$. The theorem is proved.

We know that $\operatorname{Th}\left(\mathbf{S}_{\text {len }}\right)$ is decidable [14]. Moreover, finiteness is definable with parameters: for $\psi(\vec{x}, \vec{y}), \psi_{\text {fin }}(\vec{y})$ is $\exists \vec{u}\left(\forall \vec{x} \psi(\vec{x}, \vec{y}) \rightarrow \exists \vec{z} \bigwedge_{i} z_{i} \prec u_{i} \operatorname{el}\left(z_{i}, x_{i}\right)\right)$. Thus:

Corollary 4.29 The safety of Boolean combinations of conjunctive queries in $\mathrm{RC}(\mathbf{S}), \mathrm{RC}\left(\mathbf{S}_{\mathrm{left}}\right), \mathrm{RC}\left(\mathbf{S}_{\mathrm{len}}\right), \mathrm{RC}\left(\mathbf{S}_{\mathrm{reg}}\right)$ and $\mathrm{RC}\left(\mathbf{S}_{\mathrm{reg}, \mathrm{left}}\right)$ is decidable.

Table 2 summarizes the results of the section.

\section{Conclusion}

There has been significant interest in theoretical computer science in understanding the structure of the regular languages, and in identifying subclasses of the regular languages that have special properties [67, 65]. Our work can be seen as an extension of this program, where we consider subclasses of the regular $n$-ary relations rather than the regular sets. In our approach, however, we do not focus on properties that hold of one particular regular relation by itself, but rather look at some desirable properties of a whole algebra of relations within the structure $\mathbf{S}_{\text {len }}$.

We have shown a sharp contrast between the behavior of the full algebra of regular relations of $\mathbf{S}_{\text {len }}$, and those of various submodels such as $\mathbf{S}, \mathbf{S}_{\text {left }}, \mathbf{S}_{\text {reg }}$, and $\mathbf{S}_{\text {reg,left }}$. We show that the latter are more tractable in many respects. Furthermore, we show that the behavior of an algebra of relations is not at all determined by the one-dimensional sets (subsets of $\Sigma^{*}$ ) in the algebra: for example, one can have fairly complex binary relations definable, yet still maintain 


\begin{tabular}{|c|c|c|c|c|c|}
\hline Model & Data complexity & $\begin{array}{c}\text { Data complexity } \\
\text { of generic queries }\end{array}$ & $\begin{array}{c}\text { Effective syntax } \\
\text { for safe queries }\end{array}$ & $\begin{array}{c}\text { Relational } \\
\text { algebra }\end{array}$ & Safety of CQ \\
\hline \hline $\mathrm{RC}(\mathbf{S})$ & $\mathrm{AC}^{0}$ & $\mathrm{FO}(<)$ & yes & yes & decidable \\
\hline $\mathrm{RC}\left(\mathbf{S}_{\text {len }}\right)$ & $\mathrm{PH}$ & $\mathrm{AC}^{0}$ & yes & yes & decidable \\
\hline $\mathrm{RC}\left(\mathbf{S}_{\text {left }}\right)$ & $\mathrm{AC}^{0}$ & $\mathrm{FO}(<)$ & yes & yes & decidable \\
\hline $\mathrm{RC}\left(\mathbf{S}_{\text {reg }}\right)$ & $\mathrm{NC}^{1}$ & $\mathrm{FO}(<)$ & yes & yes & decidable \\
\hline $\mathrm{RC}\left(\mathbf{S}_{\text {reg,left }}\right)$ & $\mathrm{NC}^{1}$ & $\mathrm{FO}(<)$ & yes & yes & decidable \\
\hline $\mathrm{RC}_{\text {concat }}$ & undecidable & undecidable & no & no & undecidable \\
\hline
\end{tabular}

Table 2: Summary of results on query languages

the property that all definable subsets of $\Sigma^{*}$ are star-free. Figure 1 summarizes the relationships between the star-free and regular algebras we considered here.

We have also studied extensions of the standard relational calculus with various sets of string operations. We were interested in languages that were not computationally complete, but rather shared the attractive complexity-theoretic and static analysis properties of relational calculus.

The language $\mathrm{RC}(\mathbf{S})$ can be seen as a nice foundation over which other languages should be built. It covers the most rudimentary string operations, but its expressive power is quite limited. The extension $\operatorname{RC}\left(\mathbf{S}_{\text {len }}\right)$ is too powerful (but still not computationally complete). We therefore considered the languages in between $\mathrm{RC}\left(\mathbf{S}_{\text {left }}\right), \operatorname{RC}\left(\mathbf{S}_{\text {reg }}\right), \operatorname{RC}\left(\mathbf{S}_{\text {reg,left }}\right)$ - that can express some important operations found in $\operatorname{RC}\left(\mathbf{S}_{\text {len }}\right)$, but still have low data complexity. All the calculi have effective syntax for safe queries, and corresponding relational algebras.

A key question is how many relations one can add to the models $\mathbf{S}_{\text {left }}$ or $\mathbf{S}_{\text {reg }}$ and still have the attractive properties like QE, finite VC-dimension, and a nicely-behaved relational calculus. Is there a model that is somehow maximal with respect to these properties? We would very much like to know the answer to this question. There are also several natural candidate models that would seem amenable to the approach taken here, and where one would expect the same results to go through: for example, if one allows the operation of concatenating a fixed sequence "in the middle" of a string, rather than on the left or on the right, is the resulting model still tractable?

Acknowledgments We thank Wolfgang Thomas, Scott Weinstein, Emmanuel Waller, and Jan Van den Bussche for fruitful discussions on the subject, and the anonymous referees for numerous helpful comments.

\section{References}

[1] S. Abiteboul, R. Hull and V. Vianu. Foundations of Databases. Addison-Wesley, 1995.

[2] M. Ajtai. $\Sigma_{1}^{1}$ formulae on finite structures. Annals of Pure and Applied Logic, 24 (1983), 1-48.

[3] M. Ajtai, R. Fagin and L. Stockmeyer. The closure of monadic NP. JCSS 60(3): 660-716 (2000).

[4] D. Angluin, D. N. Hoover. Regular prefix relations. Mathematical Systems Theory 17(3),167-191,1984.

[5] M. Anthony and N. Biggs. Computational Learning Theory. Cambridge Univ. Press, 1992.

[6] A. Atserias, Ph. Kolaitis. First-order logic vs. fixed-point logic in finite set theory. In LICS'98, pages $275-284$.

[7] D.A. Barrington, N. Immerman, H. Straubing. On uniformity within $N C^{1}$. JCSS, 41:274-306,1990.

[8] O. Belegradek, A. Stolboushkin, M. Taitslin. Extended order-generic queries. Annals of Pure and Applied Logic 97 (1999), 85-125.

[9] M. Benedikt, G. Dong, L. Libkin, L. Wong. Relational expressive power of constraint query languages. Journal of the ACM 45 (1998), 1-34. 
[10] M. Benedikt, L. Libkin. Relational queries over interpreted structures. Journal of the ACM 47 (2000), 644-680.

[11] M. Benedikt, L. Libkin. Safe constraint queries. SIAM J. Comput. 29 (2000), 1652-1682.

[12] M. Benedikt, L. Libkin, T. Schwentick, L. Segoufin. A model-theoretic approach to regular string relations. In LICS'01, pages 431-440.

[13] M. Benedikt, L. Libkin, T. Schwentick, L. Segoufin. String operations in query languages. In PODS'O1, pages 183-194.

[14] A. Blumensath and E. Grädel. Automatic structures. In LICS'00, pages 51-62.

[15] A. Blumer, A. Ehrenfeucht, D. Haussler, M. Warmuth. Learnability and the Vapnik-Chervonenkis dimension. Journal of the ACM 36 (1989), 929-965.

[16] N. Bjørner. Integration of Decision Procedures in Temporal Verification. PhD Thesis, Stanford University, 2000.

[17] A. Bonner and G. Mecca. Sequences, datalog, and transducers. JCSS 57 (1998), 234-259.

[18] A. Bonner and G. Mecca. Querying string databases with transducers. In DBPL'97, pages 118-135.

[19] V. Bruyère, G. Hansel, C. Michaux, R. Villemaire. Logic and p-recognizable sets of integers. Bull. Belg. Math. Soc. 1 (1994), 191-238.

[20] J.R. Büchi. Weak second-order arithmetic and finite automata. Zeit. Math. Logik Grundl. Math. 6 (1960), 66-92.

[21] C.C. Chang and H.J. Keisler Model Theory. North Holland, 1990.

[22] G. Cherlin and F. Point. On extensions of Presburger arithmetic. In Proc. 4th Easter Model Theory Conf., Humboldt Univ. Berlin, 1986.

[23] H. Comon, R. Treinen. The first-order theory of lexicographic path orderings is undecidable. TCS 176 (1997), 67-87.

[24] M. Consens and T. Milo. Algebras for querying text regions: expressive power and optimization. JCSS 57 (1998), 272-288.

[25] E. Dantsin, A. Voronkov. Expressive power and data complexity of query languages for trees and lists. In PODS'2000, pages 157-165.

[26] L. Denenberg, Y. Gurevich and S. Shelah. Definability by constant-depth polynomial-size circuits. Information and Control 70 (1986), 216-240.

[27] H.-D. Ebbinghaus and J. Flum. Finite Model Theory. Springer Verlag, 1995.

[28] A. Ehrenfeucht. An application of games to the completeness problem for formalized theories. Fund. Math., 49:129-141, 1961.

[29] C. Elgot and J. Mezei. On relations defined by generalized finite automata. IBM J. Res. Develop. 9 (1965), 47-68.

[30] D. Epstein et al. Word Processing in Groups. Jones and Bartlett Publ., 1992.

[31] M. Fischer and M. Rabin. Super-exponential complexity of Presburger arithmetic. SIAM-AMS Proceedings 7 , 27-41 (1974).

[32] J. Flum and M. Ziegler. Pseudo-finite homogeneity and saturation. J. Symb. Logic 64 (1999), 1689-1699.

[33] R. Fraïssé. Sur quelques classifications des systèmes de relations. Publ. Sci. Univ. Alger. Sér. A, 1:35-182, 1954. 
[34] C. Frougny and J. Sakarovitch. Synchronized rational relations of finite and infinite words. TCS 108 (1993), 45-82.

[35] M. Furst, J. Saxe, M. Sipser. Parity, circuits, and the polynomial-time hierarchy. Math. Systems Theory 17 (1984), 13-27.

[36] S. Ginsburg and X.S. Wang. Pattern matching by rs-operations: toward a unified approach to querying sequenced data. In PODS'92, pages 293-300.

[37] E. Grädel and Y. Gurevich. Metafinite model theory. Information and Computation 140 (1998), $26-81$.

[38] G. Grahne and M. Nykänen. Safety, translation and evaluation of alignment calculus. In Proc. of the First East-European Symp. on Advances in Databases and Information Systems (ADBIS'97), 295-304, 1997.

[39] G. Grahne, M. Nykänen, E. Ukkonen. Reasoning about strings in databases. JCSS 59 (1999), 116-162.

[40] G. Grahne, E. Waller. How to make SQL stand for string query language. In Proceedings of DBPL'99, Springer LNCS vol. 1949, 2000, pages 61-79.

[41] P. Gulutzan and S. Pelzer. SQL-99 Complete, Really. R\&D Books, 1999.

[42] R. Hakli, M. Nykänen, H. Tamm, and E. Ukkonen. Implementing a declarative string query language with string restructuring. In $P A D L^{\prime} 99$, pages 179-195.

[43] D. Harel. Towards a theory of recursive structures. In MFCS'98, pages 36-53.

[44] M. Hodges. Model Theory. Cambridge, 1993.

[45] B. Hodgson. Décidabilité par automate fini. Ann. Sc. Math. Québec 7(1) (1983), 39-57.

[46] O. Ibarra, J. Su. A technique for proving decidability of containment and equivalence of linear constraint queries. JCSS 59 (1999), 1-28.

[47] N. Immerman. Descriptive Complexity. Springer, 1999.

[48] B. Khoussainov and A. Nerode. Automatic presentations of structures. In LCC'94, pages 367-392.

[49] Ph. Kolaitis and M. Vardi. Fixpoint logic vs. infinitary logic in finite-model theory. In LICS'92, pages 46-57.

[50] G. Kuper, L. Libkin, J. Paredaens, editors. Constraint Databases. Springer, 2000.

[51] M. C. Laskowski. Vapnik-Chervonenkis classes of definable sets. J. London Math. Soc., 45:377-384, 1992.

[52] H. Läuchli and C. Savioz. Monadic second order definable relations on the binary tree. J. Symb. Logic 51(1) (1987), 219-226.

[53] A. Maĺcev. On the elementary theories of locally free universal algebras. Soviet Math. Doklady 2 (1961), 768771.

[54] R. McNaughton and S. Papert. Counter-Free Automata. MIT Press, 1971.

[55] C. Michaux, R. Villemaire. Open questions around Büchi and Presburger arithmetics. In Logic: From Foundations to Applications, Oxford Univ. Press, 1996, pages 353-383.

[56] F. Neven and J. Van den Bussche. Expressiveness of structured document query languages based on attribute grammars. Journal of the ACM, 49(1): 56-100 (2002).

[57] C. H. Papadimitriou. Computational Complexity Addison-Wesley, 1994. 
[58] M. Rabin. Weakly definable relations and special automata. In Mathematical Logic and Foundations of Set Theory, North Holland, Amsterdam, 1970, pages 1-23.

[59] A. Rajasekar. String-oriented databases. SPIRE/CRIWG'99, pages 158-167.

[60] T. Rybina, A. Voronkov. A decision procedure for term algebras with queues. ACM TOCL 2(2): 155-181 (2001).

[61] A. Salomaa. Formal Languages. Academic Press, 1973.

[62] T. Schwentick. On diving in trees. In Proceedings of the 23rd Symposium on Mathematical Foundations of Computer Science (MFCS 2000), Bratislava, 2000, pages 660-669, 2000.

[63] S. Shelah. Stability, the f.c.p., and superstability. Ann. of Math. Logic 3 (1971), 271-362.

[64] A. Stolboushkin, M. Taitslin. Finite queries do not have effective syntax. Information and Computation, 153(1) (1999), 99-116.

[65] H. Straubing. Finite Automata, Formal Logic, and Circuit Complexity. Birkhäuser, 1994.

[66] W. Thomas. Infinite trees and automaton-definable relations over $\omega$-words. TCS 103 (1992), 143-159.

[67] W. Thomas. Languages, automata, and logic. Handbook of Formal Languages, Vol. 3, Springer, 1997.

[68] K. Venkataraman. Decidability of the purely existential fragment of the theory of term algebras. Journal of the ACM 34 (1987), 492-510. 